\title{
WestVirginiaUniversity
}

THE RESEARCH REPOSITORY @ WVU

Graduate Theses, Dissertations, and Problem Reports

2019

\section{Description of motor control using inverse models}

Anton Sobinov

ansobinov@mix.wvu.edu

Follow this and additional works at: https://researchrepository.wvu.edu/etd

Part of the Control Theory Commons, Musculoskeletal System Commons, Numerical Analysis and Scientific Computing Commons, Orthotics and Prosthetics Commons, and the Other Rehabilitation and Therapy Commons

\section{Recommended Citation}

Sobinov, Anton, "Description of motor control using inverse models" (2019). Graduate Theses,

Dissertations, and Problem Reports. 4073.

https://researchrepository.wvu.edu/etd/4073

This Dissertation is protected by copyright and/or related rights. It has been brought to you by the The Research Repository @ WVU with permission from the rights-holder(s). You are free to use this Dissertation in any way that is permitted by the copyright and related rights legislation that applies to your use. For other uses you must obtain permission from the rights-holder(s) directly, unless additional rights are indicated by a Creative Commons license in the record and/ or on the work itself. This Dissertation has been accepted for inclusion in WVU Graduate Theses, Dissertations, and Problem Reports collection by an authorized administrator of The Research Repository @ WVU.

For more information, please contact researchrepository@mail.wvu.edu. 


\title{
Description of motor control using inverse models
}

\author{
Anton Sobinov \\ Dissertation submitted to the School of Medicine \\ at West Virginia University \\ In partial fulfillment of the requirements for the degree of \\ Doctor of Philosophy in \\ Neuroscience
}
Sergiy Yakovenko, PhD, Chair
Lee E Fisher, PhD
Valeriya Gritsenko, PhD
James W Lewis, PhD
Casian Pantea, PhD

Neuroscience Graduate Program

Morgantown, WV

2019

Keywords: computational neuroscience, motor control, myoelectric prosthetics, musculoskeletal modeling

Copyright 2019 Anton Sobinov 


\title{
Abstract \\ Description of motor control using inverse models
}

\author{
Anton Sobinov
}

Humans can perform complicated movements like writing or running without giving them much thought. The scientific understanding of principles guiding the generation of these movements is incomplete. How the nervous system ensures stability or compensates for injury and constraints - are among the unanswered questions today. Furthermore, only through movement can a human impose their will and interact with the world around them. Damage to a part of the motor control system can lower a person's quality of life. Understanding how the central nervous system (CNS) forms control signals and executes them helps with the construction of devices and rehabilitation techniques. This allows the user, at least in part, to bypass the damaged area or replace its function, thereby improving their quality of life.

CNS forms motor commands, for example a locomotor velocity or another movement task. These commands are thought to be processed through an internal model of the body to produce patterns of motor unit activity. An example of one such network in the spinal cord is a central pattern generator (CPG) that controls the rhythmic activation of synergistic muscle groups for overground locomotion. The descending drive from the brainstem and sensory feedback pathways initiate and modify the activity of the CPG. The interactions between its inputs and internal dynamics are still under debate in experimental and modelling studies. Even more complex neuromechanical mechanisms are responsible for some non-periodic voluntary movements. Most of the complexity stems from internalization of the body musculoskeletal (MS) system, which is comprised of hundreds of joints and muscles wrapping around each other in a sophisticated manner. Understanding their control signals requires a deep understanding of their dynamics and principles, both of which remain open problems.

This dissertation is organized into three research chapters with a bottom-up investigation of motor control, plus an introduction and a discussion chapter. Each of the three research chapters are organized as stand-alone articles either published or in preparation for submission to peer-reviewed journals. Chapter two introduces a description of the MS kinematic variables of a human hand. In an effort to simulate human hand motor control, an algorithm was defined that approximated the moment arms and lengths of 33 musculotendon actuators spanning 18 degrees of freedom. The resulting model could be evaluated within 10 microseconds and required less than $100 \mathrm{~KB}$ of memory. The structure of the approximating functions embedded anatomical and functional features of the modelled muscles, providing a meaningful description of the system. The third chapter used the developments in musculotendon modelling to obtain muscle activity profiles controlling hand movements and postures. The agonist-antagonist coactivation mechanism was responsible for producing joint stability for most degrees of freedom, similar to experimental observations. Computed muscle excitations were used in an offline control of a myoelectric prosthesis for a single subject. To investigate the higher-order generation of control signals, the fourth chapter describes an analytical model of CPG. Its parameter space was investigated to produce forward locomotion when controlled with a desired speed. The model parameters were varied to produce asymmetric locomotion, and several control strategies were identified. Throughout the dissertation the balance between analytical, simulation, and phenomenological modelling for the description of simple and complex behavior is a recurrent theme of discussion. 


\section{Acknowledgements}

I would like to thank many people that helped me throughout PhD graduate school.

First, I would like to thank my advisor, Dr. Sergiy Yakovenko, for helping me transition between very different fields of research and cultures. I have learned a lot from you about research, laboratory management, mentoring, writing, publishing, collaboration, critical thinking and philosophy of science. I have grown so much from the experience of working with you.

I am very thankful to my committee members, Dr. Valeriya Gritsenko, Dr. Lee E Fisher, Dr. James Lewis, Dr. Casian Pantea, for the help, suggestions and discussions that offered me their unique professional view of research.

Thank you, past and present members of the NEL and NERL laboratories for the help, discussion and company. Especially, I would like to acknowledge Matthew Boots, Russell Hardesty, Brad Pollard, Lisa Kogan, Kiril Tuntevski, Trevor Moon, Dr. Erienne Olesh, Theresa Boots, Ariel Thomas.

I would like to thank the RNEL laboratory at Pittsburgh University with whom we worked on DARPA HAPTIX project for the countless project meetings and discussions that we had over the years. I would like to specially acknowledge Dr. Robert A Gaunt, Dr. Lee E Fisher, Dr. Jennifer L Collinger, Dr. Douglas J Weber, Dr. Michael Boninger, Dr. Misagh Mansouri, Dr. Santosh Chandrasekaran, Carl Beringer, Tyler Simpson, David Weir.

I would like to thank Biomedical Sciences Program and Department of Neuroscience for teaching biological research to someone without biological background. Especially, I would like to acknowledge the current and previous heads of the program and the department: Dr. Fred Minnear, Dr. Lisa Salati, Dr. Richard Dey, Dr. Bernard Schreurs, Dr. Randy Nelson, and all the professors who answered my endless questions.

In the end, I would like to thank all friends that stayed by me and kept contact though different continents, time zones and life paths. 


\section{Table of Contents}

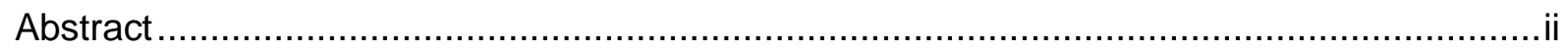

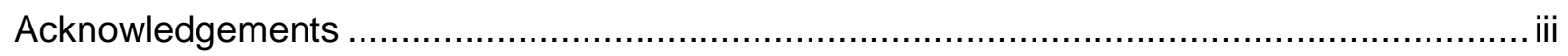

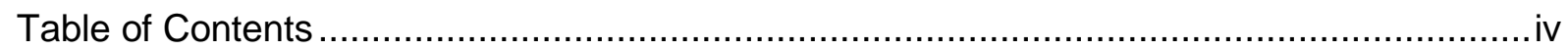

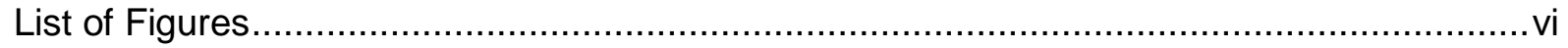

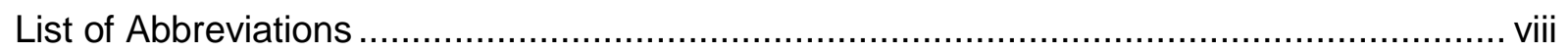

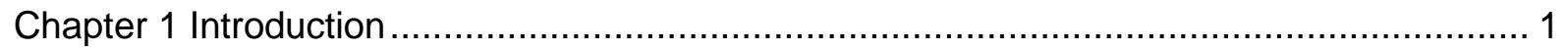

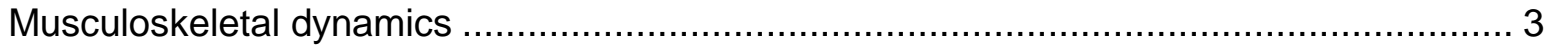

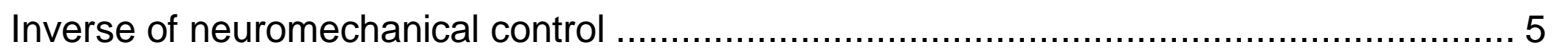

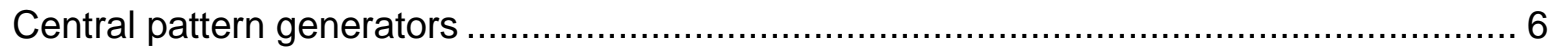

Chapter 2 Musculoskeletal transformation described with autogenerating multidimensional

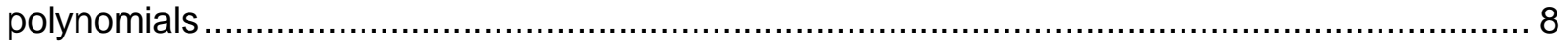

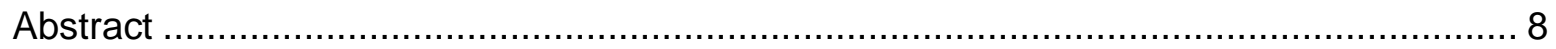

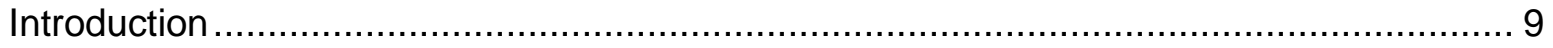

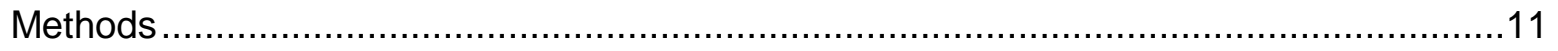

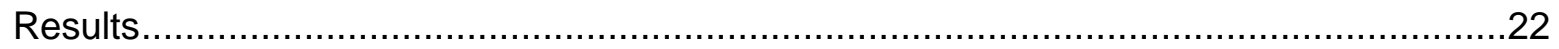

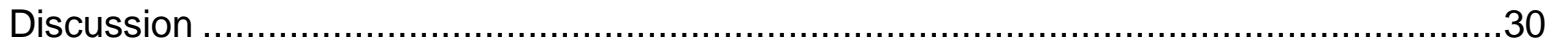

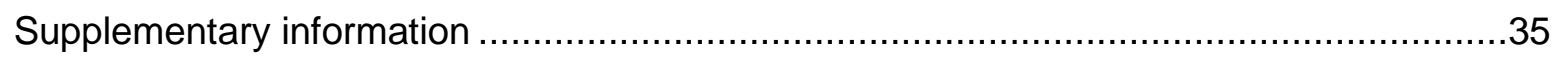

Chapter 3 Muscle excitation profiles in complex musculoskeletal model of human hand with

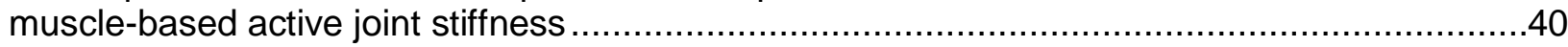

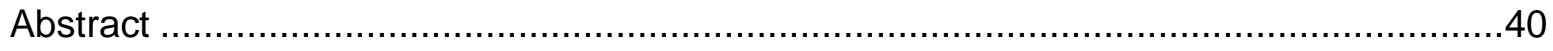

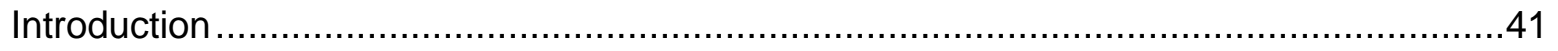

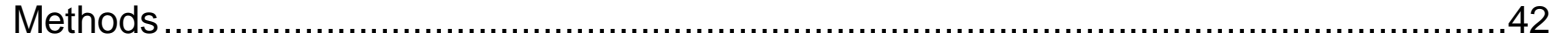

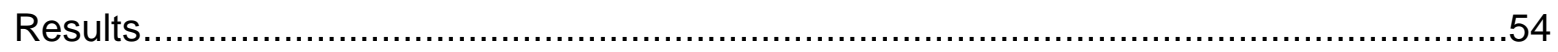

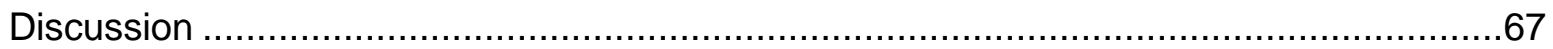

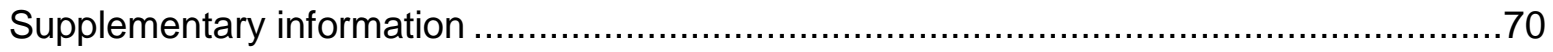

Chapter 4 Model of a bilateral Brown-type central pattern generator for symmetric and asymmetric locomotion 


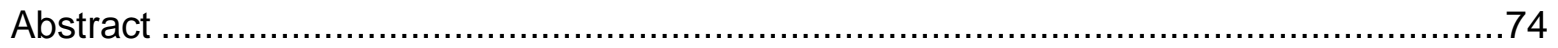

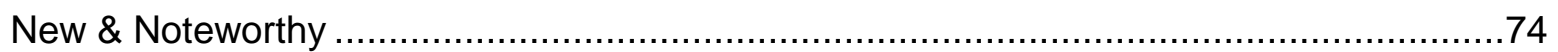

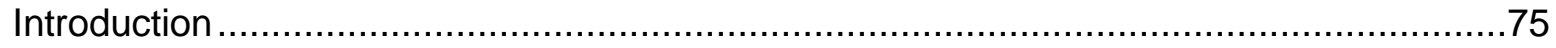

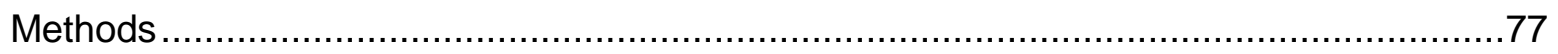

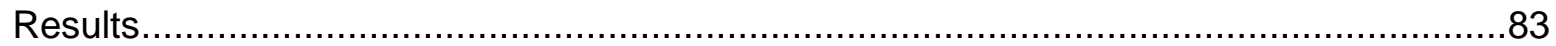

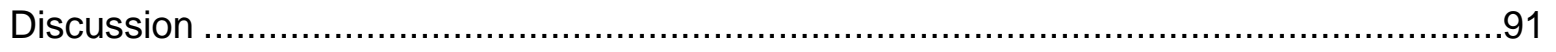

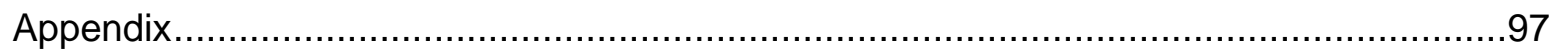

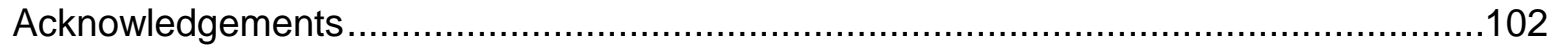

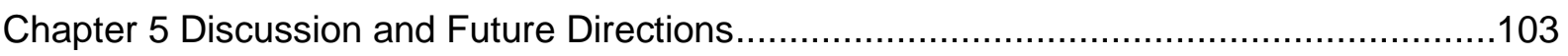

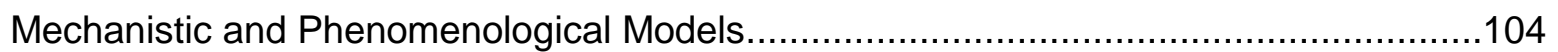

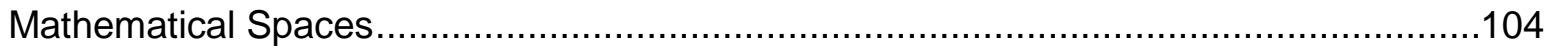

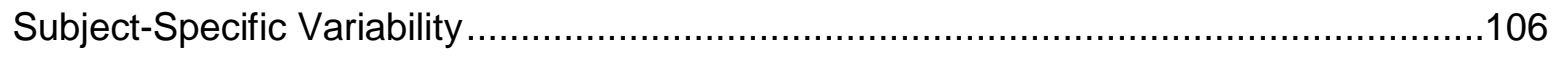

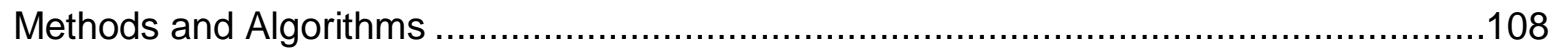

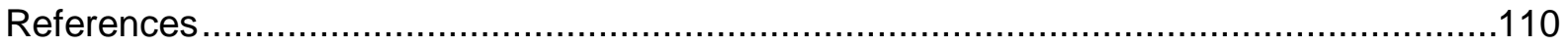

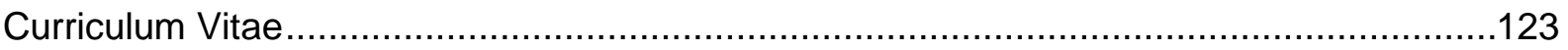




\section{List of Figures}

Figure 2-1. Upper-limb representation in OpenSim.................................................12

Figure 2-2. The example of kinematic approximation for extensor carpi ulnaris muscle.......14

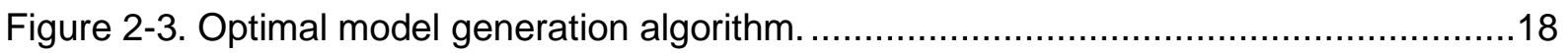

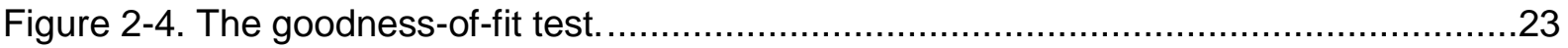

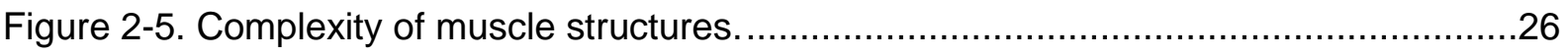

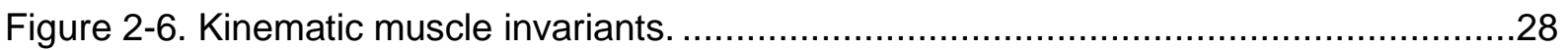

Figure 2-7. The structural and functional information embedded in muscle invariants. ........29

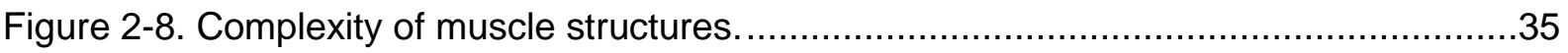

Figure 2-9. Similarity of muscle structures using Similarity Index.................................36

Figure 3-1. Schematic of the steps in forward (black) and inverse (red) control of the

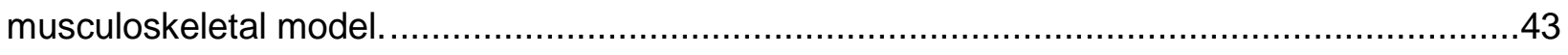

Figure 3-2. Schematic of active muscle stiffness for one DOF and two muscles.................51

Figure 3-3. Error in torque reconstruction by CMEs.................................................55

Figure 3-4. Change in CMEs levels with change in desired stiffness. ..........................56

Figure 3-5. Example of CME profiles with different levels of desired stiffness...................58

Figure 3-6. Desired $\left(K^{\star}\right)$ and observed $(K)$ levels of stiffness in the inverse solutions..........59

Figure 3-7. Agonist-antagonist coactivation is partially responsible for the joint stiffness.....61

Figure 3-8. Dynamic forward simulation of a grasping movement controlled by CMEs. ........63

Figure 3-9. Control of movements using CMEs. ....................................................64

Figure 3-10. Control of dynamic movements using recorded EMG ............................66

Figure 4-1. Schematic of the bilateral locomotor CPG model....................................78 
Figure 4-2. Experimental and simulated locomotor phase duration characteristic.

Figure 4-3. The comparison of analytical and numerical solutions. ............................... 84

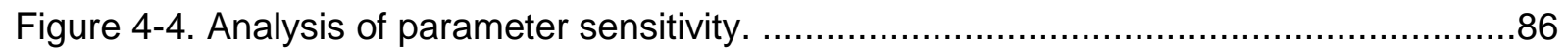

Figure 4-5. The relationship between the simulated CPG command signal to each limb and

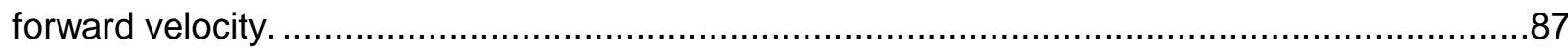

Figure 4-6. External inputs generate asymmetric gait in the model...............................88

Figure 4-7. Intrinsic parameters generate asymmetric gait in the model. ........................90

Figure 4-8. Schematic representation of multiple CPG configurations for steering..............94

Figure 4-9. Solutions with the varied manipulations of the optimization algorithm.............101 


\section{List of Abbreviations}

AIC: Akaike Information Criterion

AICc: corrected Akaike information criterion

AU: arbitrary units

BFGS: Broyden-Fletcher-Goldfarb-Shanno algorithm

cDOFs: controlled degrees of freedom

CME: computed muscle excitation

CMC: computed muscle contraction

CNS: central nervous system

COBYLA: constrained approximation by linear approximation

$\mathrm{CP}$ : constrained polynomials

CPG: central pattern generator

CPU: central processing unit

CS: cubic splines

DBS: deep brain stimulation

DOF: degree of freedom

ECC: excitation-contraction coupling

EMG: electromyogram

FES: functional electrical stimulation

GB: gigabyte

$\mathrm{GHz}$ : gigahertz

HMI: human-machine interface

ICMS: intracranial microstimulation

ISMS: intraspinal microstimulation

KB: kilobyte

MA: moment arm

MCP: metacarpophalangeal 
MEE: maximum expected error

MLE: maximum likelihood estimation

MLR: mesencephalic locomotor region

MS: musculoskeletal

MSD: musculoskeletal dynamics

MVC: maximum voluntary contraction

NU: normalized units

ODE: ordinary differential equation

PC: principle component

PCA: principle component analysis

RAM: random access memory

RMSE: root mean square error

ROM: range of motion

SE: standard error

SI: similarity index

SSD: solid state drive

TMS: transcranial magnetic stimulation

UP: unconstrained polynomials 


\section{Chapter 1 Introduction}

Voluntary movement produced by skeletal muscles is essential for every human. Damage to any part of the motor system greatly impacts quality of life in various aspects: autonomy, social interactions, physical and psychological health (Laurent et al., 2011). A wide variety of rehabilitation techniques and prosthetic devices have been developed to overcome motor impairments. In rehabilitation approaches for stroke or spinal cord injury, one major focus is on enhancing the remaining potential of the central nervous system (CNS) related to motor control or bypass the damaged area to provide a patient with new means of motor control and interactions with their environment. Many stimulation-based treatments are emerging: transcranial magnetic stimulation (TMS: Rossi and Rossini, 2004), deep brain stimulation (DBS: Schiff et al., 2007; Little et al., 2013), intracortical and intraspinal microstimulation (ICMS: Flesher et al., 2016; ISMS: Formento et al., 2018), and functional electric stimulation (FES: Alon et al., 2007). These methods are utilized to access the spared neural pathways to form neuroprostheses operated through human-machine interface (Nicolas-Alonso and Gomez-Gil, 2012). The obstacle for robust neuroprosthetic solutions is the complexity associated with human limb dynamics and its natural control (Quental et al., 2015; Tucker et al., 2015). The human body contains more than 200 bones and over 600 muscles many of which have multiple heads or insertions with different functions and separate control. All these actuators are constantly being controlled by the CNS to produce complex multi-joint movements like running, writing or playing a musical instrument. The development of novel rehabilitation techniques and prosthetics depends on unraveling the complexity of musculoskeletal systems and their control by the CNS.

Motor control is executed by a redundant system with many steps of transformation from the desired movement to its execution. Every movement can be performed with an infinite number of joint configurations; many joint configurations can be produced by a variety of muscle activation patterns; and a muscle force can be generated by activating different subsets of motor units. This 'redundancy problem' has been first noted by Bernstein when studying variation in repetitive movements in 1960s (Bernstein, 1967). The redundancy of such a system raises the question of how the CNS chooses a specific way of executing the task. That question could be reformulated as finding the correct control signal given the observed behavior, i.e., solving the inverse problem to the forward description of motor control. All possible control 
signals producing an observed behavior form the task-related subspace of the control signal space. This subspace of solutions for the inverse model is in practice constrained by the cost functions or constraints that describe the principles of movement generation, for example, metabolic cost (Alexander, 1997) or joint impedance (Stroeve, 1999; Todorov, 2004). The remaining solutions form an uncontrolled manifold, from which the CNS selects a specific control command (Scholz and Schöner, 1999; Todorov and Jordan, 2002; Valero-Cuevas et al., 2009). Repetitions of the movements lead to sampling of that manifold and observed variability. By understanding the principles which guide the selection of control commands at different levels and constrain the uncontrolled manifold, one can infer the control strategy used by the CNS.

Analysis of the control strategies in the described approach is limited by the quality of the forward and inverse models employed. They can vary from a detailed analytical representation of the internal state and mechanisms of the modelled system to phenomenological description of input and output. Phenomenological models commonly employ regression or machine learning techniques and characterize the system's behavior with minimum assumptions about its structure (Mauk, 2000). The phenomenological approach, especially when applied to complex phenomena, often produces models that are hard to analyze and dissect, and therefore have limited use for theoretical understanding of the subject. These models also have a possibility to include unwarranted hypothetical features, which hinders their capabilities to expand to additional cases or to combine and integrate with other models. Their theoretical use is to guide the development of the analytical or mechanistic models with features of their architecture. The mechanistic models are defined by the description of the interactions between their parts and therefore are transparent for analysis and inversion. The inverse formulation of the analytical model can help estimate the boundaries of the phenomena described by the model and verify the validity of each component. The quality of mechanistic models is limited by the available theoretical understanding and experimental data available for each component. Simulation models also commonly suffer from high computational complexity, hindering their use in applied devices and large-scale meta-analyses. It is common to employ approaches based on the problem at hand, considering the benefits and drawbacks of each.

One of the leading theories suggests that CNS also employs models of the body and environment for control (Wolpert et al., 1995). The use of internalized representations of limbs is often featured in studies of the relationship between neural activity and limb end-point 
movement. For example, the cortical activity can be related to acceleration (Hore and Flament, 1988), trajectory (Hocherman and Wise, 1991), target position (Alexander and Crutcher, 1990), joint configuration (Scott and Kalaska, 1995). Transformation between these modalities into muscle activity is one type of internal model; specifically, it is an inverse model. Using it, the CNS can predictively generate appropriate commands from desired trajectory to motor commands and the resulting executed trajectories that are optimal for a specific task. The internal models for common behaviors, like locomotion, can be phylogenetically conserved between species. The rhythmic activity for locomotion is produced by central pattern generators (CPGs, Marder and Calabrese, 1996), which are spinal processing networks organized in relation to the limb musculoskeletal morphology (Prochazka and Yakovenko, 2007). Both neural and musculoskeletal systems have been undergoing concurrent functional fine-tuning that resulted in the embedding of solutions to limb dynamics problem within the neural code (Geyer and Herr, 2010). Similarities in the control of locomotion and reaching at the primary motor cortex (Yakovenko and Drew, 2015) sugests that all levels of control employ internalized models of body segments.

The description of periodic and non-periodic movement control remains incomplete in large part because the high mechanical dimensionality of human body has hindered the development of analytical tools. Thus, the critical need is first to develop realistic MS models for investigating the relation between neural control mechanisms and MS functions across representative behaviors. Second, to use these models to estimate the neural control patterns. Third, to analyze the generation of these patterns for a specific task. The rest of the Introduction will provide background for motor control models analyzed in this dissertation from the lowest level of biomechanics to spinal motor unit excitations to control of central pattern generators.

\section{Musculoskeletal dynamics}

The reliability of the identification of the control principles from inverse models is limited by the quality of the models employed. These are, in turn, limited by the quality and performance of the forward models. Invalid or too simplistic models lead to incorrect results of simulations (Quental et al., 2015). High computational load of the complex models limits their use in research or rehabilitative devices (Delp et al., 2007; Sartori et al., 2012).

A structural model of a musculoskeletal (MS) system consists of the geometrical description of segments and joints with paths that muscles travelling between points of attachment. MS 
systems of mammalian bodies contain hundreds of muscles and dozens of joints, with multiple axes of rotation, or degrees of freedom (DOFs). Muscles have multiple heads with multiple points of attachment, cross multiple DOFs, and wrap around each other in a posture-dependent way. Simulation of that behavior is a very complex task (Delp et al., 2007) and is usually not feasible online except in very simple models. To negate that limitation, many researchers employ approximations to the simulated kinematic variables (Menegaldo et al., 2004; Sartori et al., 2012). Although accurate, these approximations usually do not expand beyond several DOFs because of computational limitations (Sartori et al., 2012) or the manual nature of their structure (Menegaldo et al., 2004). Model validation and limited availability of data additionally reinforces limitations to small and restricted body segments and ranges of postures. However, recent research started to tackle the problem of merging datasets and developing extensive verification procedures for the whole physiological range of motion (Goislard De Monsabert et al., 2018; Boots et al., 2019). Complex models with many body segments and correctly modelled musculotendon actuators are starting to be available for analysis and application.

These models allow the study of the embedded latent dynamical properties within the MS anatomy that has been previously hypothesized to have evolved to enable and stabilize various movements. For example, the moment arms of distal extensors produce abduction moments stabilizing the medio-lateral travel of the center of mass during the stance phase of locomotion; and the moment arms of distal flexors produce adduction moments ensuring medial foot placement at the end of swing (Lawrence et al., 1993). The medial placement has been shown to be more energy-efficient than the lateral placement, associated with the wide stance (Donelan et al., 2001). The passive dynamics of the MS system provide compensation for unexpected terrain during human hopping (van der Krogt et al., 2009). There may be additional details hidden in the complexity of musculoskeletal dynamics (MSD) that stabilize both upper and lower limbs. A large-scale analysis of the structure of MS variables or their representative functions has a potential to reveal these motifs and behaviors.

Chapter 2 describes a novel autogenerating approximation algorithm for MS kinematic variables that utilizes a differential relationship between the muscle lengths and moment arms. The ability of this algorithm to scale for large models and compare its performance against other approximation methods is evaluated. The structure of the optimal approximating polynomials is investigated for presence of structural and functional information about the muscles. 


\section{Inverse of neuromechanical control}

The problem of inverting the computation through MS system and obtaining the motor commands in the form of muscle contraction, together with the activity of motoneurons and other spinal and cortical networks, has been an open question for more than fifty years. It finds its source in the redundancy of MS systems in relation to the task, which was first described by Bernstein (Bernstein, 1967). The same reaching task can be solved by an infinite number of joint configurations; and a joint trajectory can be traced using different subsets of motor signals.

Understanding the principles behind the control signals profoundly influences our understanding of the CNS (Churchland et al., 2012; Lillicrap and Scott, 2013; Ambike et al., 2016). These developments have a potential to lead to novel stimulation-based treatments, neuroprosthetics and other assistive devices. Stimulation of the spinal cord activity with accordance to the natural dynamics of the spinal circuits allowed a paralyzed patient to walk (Formento et al., 2018). Cortical implants already allowed a paralyzed human to control a prosthetic hand (Downey et al., 2017) and perceive touch (Flesher et al., 2016). Most of these control algorithms use machine learning to provide the desired behaviors, but they have a potential to be improved with better grasp of the underlying dynamics of the respective circuits. Improved understanding can come in a form of simulated analytical models of the motor control.

The majority of myoelectric prosthetics also commonly employ phenomenological models. The models range from a simple regression (Scott and Parker, 1988; Ison and Artemiadis, 2014) to complex pattern recognition and learning algorithms (Graupe et al., 1977; Englehart and Hudgins, 2003; Nieveen et al., 2017; Resnik et al., 2018). While the phenomenological approach provides control, there are several drawbacks common to such architecture. One of them is the need for a long training period, unique for each subject/patient and covering several months of often frustrating repetitive tasks. Another common problem is the generalizability of control between postures. Muscle activity profiles for the same movement change when the arm is oriented vertically or horizontally, which is a complex invariant that needs to be extracted by the control algorithm. A biomimetic approach has potential to handle these problems. There are many views on biomimetic and biomorphic approaches in control. In prosthetics it is commonly defined by a simulation of the intact MS system to calculate the desired movements. The benefit of this approach is the scalability and transparency of the control mechanism that requires minimal or no training period (Crouch and Huang, 2016). However, this control approach has 
critical dependencies on the correct identification of recorded commands (via electromyography, electroneurography or other methods) and estimation of unobserved commands - the activity of other muscles. The former is especially hard with amputees and is usually addressed by a physician and visual analysis of the recorded signals (Cappellari et al., 2018). The latter is sometimes addressed by employment of synergies within the controller, which supply the activity to the simulated muscles that are not recorded based on known correlations in the activity of the muscles in different tasks (Berger and d'Avella, 2014). The employed synergies are usually generic and ignore subject-specific variability. Biomimetic decoding of the descending command from the movement has a potential to simplify the control of prosthetic limbs by closing the gap between the recorded signals and the desired movement.

Chapter 3 describes a detailed forward and inverse neuromechanical model of the human hand. The inverse solutions for this model are obtained for a set of movements and postures that include dynamic control and stability of wrist, thumb and fingers joints. Mechanical stability of the system is modulated by an additive cost function in the inverse model. The mechanism responsible for the generation of stiffness in joints is then investigated to identify voluntary control strategies. A method of using the computed muscle excitations as a part of a biomimetic controller is assessed.

\section{Central pattern generators}

Central pattern generators (CPGs) for locomotion are specialized neural elements in the spinal cord that control the generation of rhythmic patterns for locomotor behaviors (Grillner and Zangger, 1975; Marder and Calabrese, 1996). In mammals, they can be initiated and controlled by sensory signals (Yakovenko, 2011; Prochazka and Ellaway, 2012) and descending drive from brainstem (Shik et al., 1966; Grillner et al., 2008). The increase in stimulation of a brainstem locomotor region in cats produces sequential switching of gaits, from walking to trotting or galloping in over-the-ground locomotion. Thus, increasing stimulation magnitude or frequency causes an increase in locomotor velocity. CPG networks have been suspected in humans (Dietz, 2003; Ivanenko et al., 2009) and spinal cord has been used as a target for patterned periodic stimulation in paraplegic patients for walking (Wagner et al., 2018). Behavior of CPG network is conserved between species and presents a ubiquitous phenomenon for analysis. 
Many models of CPGs have been developed describing different aspects of circuitry (Verzár, 1923; Taga et al., 1991; Bashor, 1998; Yakovenko et al., 2005; Rybak et al., 2006; Markin et al., 2010; Barnett and Cymbalyuk, 2014). Models commonly employ a specific neural or populational architecture and estimate the values for parameters by observing the changes in behavior in response to changes in external inputs or internal structure. This approach is a blend of analytical and simulation description of neural architecture with phenomenological estimation of parameters. With the benefits of both, it requires careful attention to the parameter space and the emergent topology.

Chapter 4 describes an analytical solution to a model of Brown-type CPG. The generation of a motor rhythm that precedes the muscle excitation patterns in response to a command signal is investigated. The computational complexity of such model is analyzed, and the benefits of an analytical solution are demonstrated. The parameter space of the model is explored to find solutions for a physiological forward-walking behavior. The solution space is used to guide a discussion of the necessary model complexity for simulation of locomotion control. Different internal configurations of the CPG that produce asymmetric (turning) behavior are analyzed and presented. 


\title{
Chapter 2 Musculoskeletal transformation described with autogenerating multidimensional polynomials
}

(this chapter is in preparation to be submitted to eLife as "Sobinov A, Matthew B, Gritsenko V, Gaunt R, et al., Yakovenko S (2019) Musculoskeletal transformation described with autogenerating multidimensional polynomials")

\begin{abstract}
Computational models of the musculoskeletal (MS) system can be used to resolve the complexity of controlling high-dimensional articulated prosthetic limbs. Similar to the use of internal models of the body by the nervous system in solving control problems, MS representations can be useful tools for controlling realistic prostheses operated by biological control signals. Precise estimation of kinematic variables is needed to estimate muscle forces and produced rotational moments. However, the implementation of accurate and fast MS computations which can be used to control a prosthetic limb in real time is a challenging problem because skeletal muscles span multiple degrees of freedom (DOF) and wrap over complex geometrical constraints. Here, we exploited the relationship between muscle length and moment arms and capitalized on the observations of their relatively simple functional dependency on posture to improve both the accuracy and economy of computations. MS kinematics were then captured by the autogenerating polynomials which used information theory to select their optimal terms. The polynomial terms were iteratively selected for 33 musculotendon actuators, each spanning up to 6 DOFs in an 18 DOF model of the human arm and hand, defined within the full physiological range of motion. Using these polynomials, muscle lengths and moment arms were accurately computed with better than real-time latencies $(<10 \mu s)$. The approximation structure was shown to scale linearly with model's complexity. Moreover, we demonstrate that both muscle structure and function correlate with specific invariant polynomial terms. The clustering of muscles with specific terms represents synergistic relationships. We propose that the novel method of describing MS mechanisms might further improve the applications of detailed and scalable models for interpreting neural computations.
\end{abstract}




\section{Introduction}

The control of a hand is a 27 degree of freedom (DOF) problem solved continuously by our neuromuscular system without perceived cognitive effort. Yet, for prosthetic applications, the current approaches, such as pattern recognition and mode switching require significant training time (Hiremath et al., 2015); moreover, the skill and cognitive load required for continuous prosthetic control increases with the number of available prosthetic DOF (Deeny et al., 2014). This phenomenon is captured by the dimensionality curse problem in movement planning, which occurs due to the increasing volume of possible solutions with the increasing number of dimensions. Recently, machine learning statistical methods have been gaining popularity in computer vision and robotic control problems of comparable complexity. In particular, deep learning algorithms are promising and outperforming the shallow varieties via the hierarchy of processing, which is a biomimetic property common to biological cortical networks (Poggio et al., 2017). This property reduces the escalation in computations with the increase in complexity of learning machines. However, the process of their fine-tuning requires large amounts of data and usually results in a black box transformation, without many transparent internal mechanisms and generates few insights into the underlying control scheme (reviewed in Lapuschkin et al., 2019). In addition, machine learning solutions often require episodic model retraining (Hermann et al., 2015), and rely on a considerable memory space for the storage of necessary parameters (Weston et al., 2014). These constraints pose significant challenges for real-time control systems for both phenomenological and mechanistic models of human hand biomechanics. Overall, this approach limits our understanding of model boundaries, the reliable domain of operation, and, importantly, the principles of the modelled system that can be tested and improved further. The use of mechanistic alternatives based on known biology may overcome these limitations.

The use of biomimetic design within the controlled devices may solve the problem of integration between the technology and biological control system. Using a biomimetic model, the challenge can be redefined to focus on specifying and implementing valid motor control theories. One such dominant theory focuses on internal models expressed within nervous system (Wolpert et al., 1998; Kawato, 1999; Körding and Wolpert, 2004) and embodies an engineering concept termed the Smith predictor (Smith, 1957). This approach uses accurate estimates of the controlled plant to overcome both nonlinear dynamics and temporal delays. Another concept is neuromechanical tuning (Prochazka and Yakovenko, 2007; Ting, 2007; 
Sreenivasa et al., 2019), which is the process taking place within the closed-loop system of neural and mechanical dynamics. To use any of these control theories, body dynamics and musculoskeletal (MS) biomechanics are essential components that require valid models (Ting et al., 2015; Blum et al., 2017) or good-enough biomimetic approximations within the design of a robotic prosthesis (Xu and Todorov, 2016). The recent use of MS models for human-machine interfaces (HMI, Crouch and Huang, 2016) shows promising results for this type of approach.

MS modelling is an important scientific tool in theoretical motor control (Berniker et al., 2009; Winter, 2009; Lillicrap and Scott, 2013) and its applications in HMI (Thorsen et al., 2001; Chadwick et al., 2009; Crouch and Huang, 2016). Models employed in research and applications constantly increase in complexity, raising their computational cost. Various taskspecific approximation methods have had limited success counteracting the rising computational demand (Menegaldo et al., 2004; Sartori et al., 2012). There is a need for an efficient way to compute MS models to allow for their continuous use in real-time environments.

MS models are usually comprised of a geometrical descriptions of each joint's degrees of freedom (DOFs) and muscles' paths around these DOFs. A muscle's action depends on the distance to the DOF axis of rotation, called moment arm, and muscle length, which alters the force produced by the muscle (Brand et al., 1975; An et al., 1984; Zajac, 1989). Calculating these MS kinematic variables in a specific posture requires computation of the shortest path between the points of attachment in the presence of objects around which a muscle wraps, like bones and other muscles (Delp et al., 2007). Software packages like OpenSim (SimTK) provide tools for computation of kinematic variables based on a 3D model of a limb or whole body. These calculations are very computationally costly and can only be performed in real time for simple models.

The complexity of the MS variables' computation has led to the development of multiple approximation methods that improve computational efficiency. Menegaldo and colleagues (Menegaldo et al., 2004) proposed a series of multidimensional polynomials describing the MS variables of human leg muscles. Later these polynomials were used to simulate the musculotendon dynamics of upper (Rankin and Neptune, 2012) and lower limbs (Chadwick et al., 2009). This approach supports very high computational performance: low requirements on the available memory and the number of mathematical operations. On the other hand, it is limited by the user-defined structure of the polynomials. The task of predicting the approximating polynomial terms gets drastically harder in more complex models, for example 
the ones that include thumb muscles (up to 7 DOFs and 7-dimensional polynomials). Additionally, relying on a subjective selection of polynomial terms can lead to losing important relationships within the MS data. Another approach developed by Sartori and colleagues (Sartori et al., 2012) emphasizes the quality of approximation and utilizes cubic splines for approximations. Albeit being computationally expensive, the ability of this approach to operate at real time has been shown in a 3-DOF per muscle model (Durandau et al., 2018). The drawback of this approach is the limited scalability: the number of spline coefficients increases exponentially with the number of DOFs that the muscle crosses and reaches 4.8 million on a 6DOF muscle. Both described methods exhibit problems with accommodating the increasing complexity of the models and severely limit the possibility of MS structure analysis.

In this study we present an information theory-based algorithm of polynomial approximation of MS kinematic variables that scales linearly with the complexity of the model. We assess the quality of the produced approximations in terms of approximation error and time of evaluation on a MS model with 33 musculotendon actuators that cross up to 6 DOFs. The structure of the produced optimal polynomials is analyzed in terms of muscle anatomy and function.

\section{Methods}

The approximation of muscle path kinematic variables consisted of three steps: $i$ ) the creation of a dataset describing muscle length and moment arm values for all physiological postures; ii) the search for a set of optimal polynomials approximating kinematic variables implemented with a physical constraint between muscle moment arms and muscle length; and iii) the validation of the produced model.

\section{Dataset}

We used a previously developed model of arm and hand to capture the relationship between muscle lengths and moment arms in all physiological postures (Gritsenko et al., 2016; Boots et al., 2019). The model contains 22 muscles described with 33 musculotendon actuators spanning 18 physiological degrees of freedom (see Table 2-3 and Table 2-4) and was implemented in OpenSim software (Delp et al., 2007) (see Figure 2-1). Similar to the previous study of Sartori et al. (Sartori et al., 2012) the values for the kinematic variables were obtained on a uniform grid with 9 points per DOF, resulting in the domain size of $9^{d}$ data points per muscle, where $d$ is the number of DOFs that a muscle crosses. For example, since the extensor 
carpi ulnaris muscle spans two DOFs (wrist flexion-extension and pronation-supination) in our dataset (radial-ulnar deviation was not simulated) its moment arms and muscle lengths were sampled in $9^{2}=81$ positions.

To compare quality for the approximations with different methods (described below), we used a dataset (total 1,023,073 points) that combined data used for the creation of the models $(674,937 ; 9$ points per DOF per muscle), and datapoints located between the points used for creation $(348,136$; 8 points per DOF per muscle).

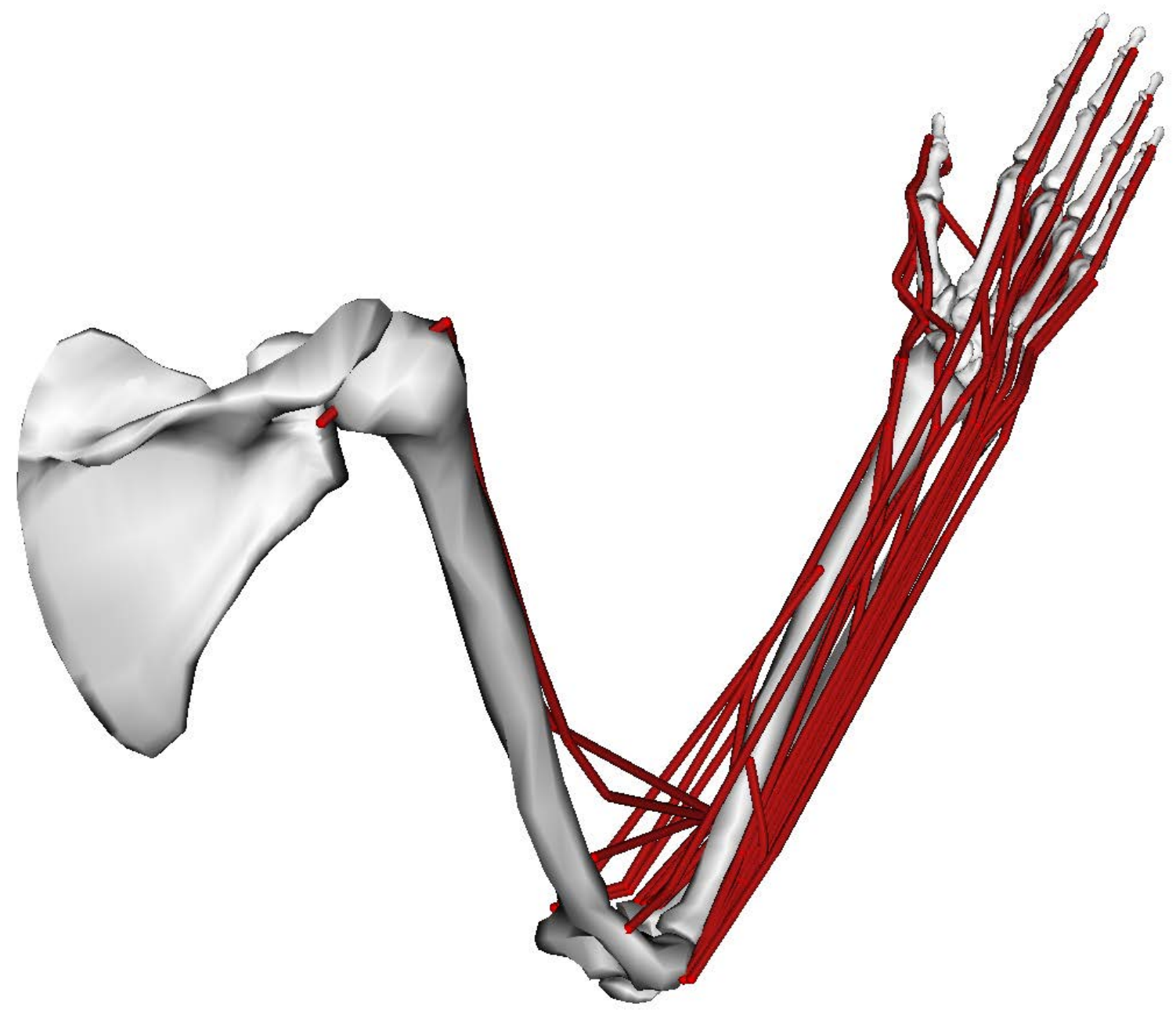

Figure 2-1. Upper-limb representation in OpenSim.

The geometry of muscle paths is shown in red for the displayed posture. 


\section{Model Structure}

Moment arms and muscle lengths were approximated with a polynomial described by Equation 2-1.

\section{Equation 2-1}

$$
f(x)=a+\sum_{p}^{\rho} \sum_{i_{1} \leq i_{2} \leq . \leq \leq i_{p}}^{d} K_{i_{1}, i_{2}, . ., i_{p}} \prod_{j}^{p} x_{i_{j}}
$$

where $a$ is an intercept, $\rho$ is the user-selected maximum of polynomial power, $d$ is the number of DOFs, $x=\left(x_{1}, \ldots, x_{d}\right)^{T}$ is the state vector for each DOF, $K$ is the multidimensional matrix of polynomial term coefficients, sum and product coefficients $(p, i$, and $j)$ iterate from 1. The polynomial structure is then defined by the non-zero values of $K$ and a parameters. For example, extensor carpi ulnaris with $\rho=4, d=2$ moment arms were described by the polynomial structures $\left(a, K_{1}, K_{2}, K_{11}, K_{12}, K_{22}, K_{111}, K_{112}, K_{122}, K_{222}, K_{1111}, K_{1112}, K_{1122}, K_{1222}\right)$ around elbow extension-flexion (e-f) and $\left(a, K_{1}, K_{2}, K_{11}, K_{12}, K_{22}, K_{111}, K_{112}, K_{122}, K_{222}, K_{1111}, K_{1112}, K_{1122}, K_{1222}, K_{2222}\right.$ ) around wrist supination-pronation (s-p) (Figure 2-2B), where indices 1 and 2 correspond to pronationsupination and flexion-extension, respectively. The difference in the structures correspond to different characteristics of muscle paths wrapping around different DOFs. 


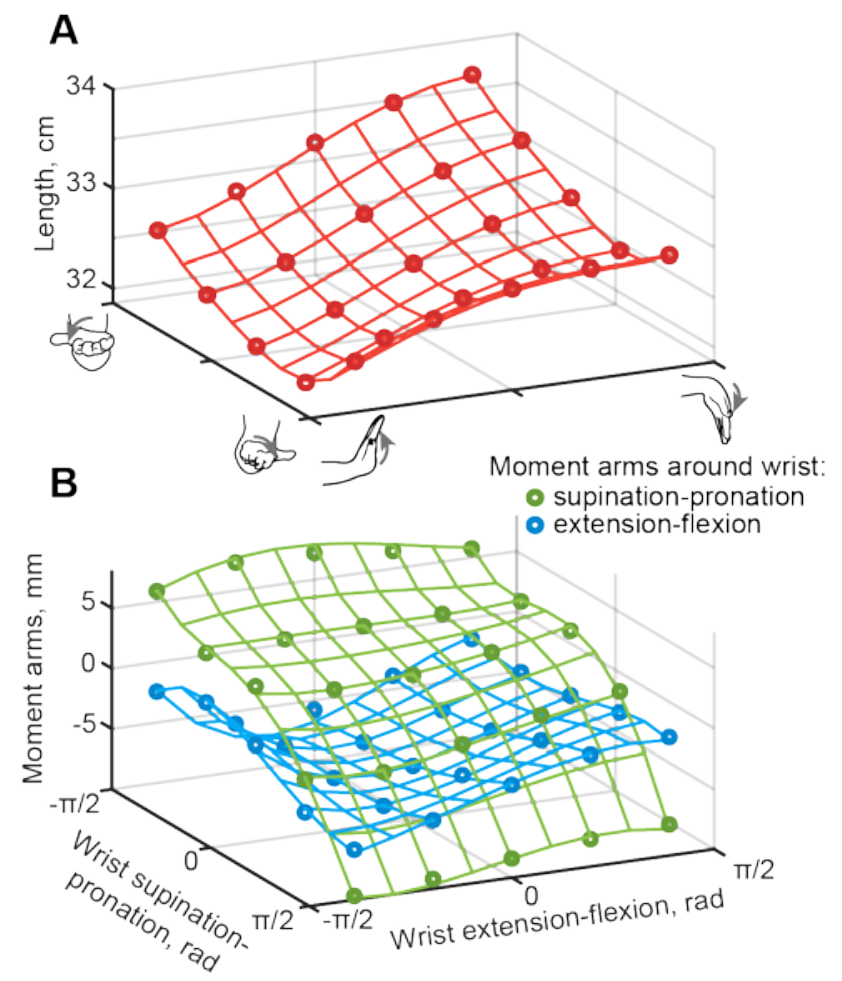

Figure 2-2. The example of kinematic approximation for extensor carpi ulnaris muscle.

A. The muscle path length is shown as a function of wrist e-f and s-p DOFs, with points from OpenSim model fitted with the continuous functions plotted as a wireframe. B. The two corresponding moment arm relationships are shown for the same domain of postures.

The accuracy of polynomial fit generally increases with the number of terms in the polynomial structure. The selection of potential candidates for expansion, $\Psi(P(x))$, contains polynomials with all terms of $P(x)$ and one additional term from the possible additional terms in a polynomial of the same power. For example, let $P(x)$ be a two-dimensional polynomial with structure $\left(a, K_{1}, K_{11}\right)$, full 2-dimensional polynomial of power 2 has a structure $\left(a, K_{1}, K_{2}, K_{11}, K_{12}, K_{22}\right)$. Then the list of potential candidates is: $\Psi(P(x))=$ $\left[\left(a, K_{1}, K_{2}, K_{11}\right) ;\left(a, K_{1}, K_{11}, K_{12}\right) ;\left(a, K_{1}, K_{11}, K_{22}\right)\right]$. The size of $\Psi(P(x))$ increases when higher power terms are required. 


\section{Model Physical Constraints}

Moment arms can be estimated as a partial differential of the muscle length in local coordinates (Brand et al., 1975; An et al., 1984):

\section{Equation 2-2}

$$
M_{i}(x)=\frac{\delta L(x)}{\delta x_{i}}
$$

where $i$ is the index of a DOF actuated by the muscle, $x_{i}$ is the coordinate of ith DOF, $M_{i}(x)$ is the posture-dependent function of the moment arm around ith DOF, $L(x)$ is the muscle length function. The kinematics of a given muscle is then captured by a single function $L(x)$ and a set of functions $\left\{M_{i}(x)\right\}$ for muscles spanning multiple DOFs.

The following algorithm finds a new function $L(x)$ and updates its set of moment arm functions $\left\{M_{i}\right\}$ in agreement with the relationship in Equation 2-2:

1. Calculate a set of intermediate muscle length polynomials $\left\{\tilde{L}_{i}(x):=\int M_{i} d x_{i}\right\}$.

2. Combine the terms of $L(x)$ and $\left\{\tilde{L}_{i}(x)\right\}: L(x):=L(x) \cup\left(\bigcup_{i}\left\{\tilde{L}_{i}(x)\right\}\right)$.

3. Differentiate analytically the polynomial $L(x)$ (Equation 2-2) to update the complimentary set of moment arm functions, $\left\{M_{i}(x)\right\}$.

4. Calculate $a$ and $K$ coefficients in $L(x)$ and $\left\{M_{i}(x)\right\}$ using the original dataset.

For example, let $x=\left(x_{1}, x_{2}\right)$, functions describing muscle length is $L=2 x_{1} x_{2}^{2}$, with moment arms $M_{1}=3 x_{1}^{3}+2$ and $M_{2}=5 x_{1} x_{2}$ were obtained from the data or an optimization routine. For muscle length, the structure describing the term $x_{1} x_{2} x_{2}$ is $K_{122}$, which is equal to 2. Similarly, $M_{1}=3 x_{1}^{3}+2$ is described by the structure $\left(K_{111}, a\right)$ and $M_{2}=5 x_{1} x_{2}$ is $\left(K_{12}\right)$. The integrals of $M_{1}, M_{2}$ in step 1 are: $L_{1}=x_{1}^{4}+2 x_{1}+$ const or structure $\left(a, K_{1}, K_{1111}\right) ; L_{2}=2.5 x_{1} x_{2}^{2}+$ const or structure $\left(a, K_{122}\right)$. In step 2, the ensemble function $L(x)$ adhering to Equation 2-2 will be $L=$ $C_{0}+C_{1} x_{1} x_{2}^{2}+C_{2} x_{1}^{4}+C_{3} x_{1}$, where $C_{i}$ are scalar coefficients in the structure $\left(a, K_{1}, K_{122}, K_{1111}\right)$. In step 3, the moment arms are $M_{1}=C_{4} x_{2}^{2}+C_{5} x_{1}^{3}+C_{6}$ or structure $\left(a, K_{22}, K_{111}\right)$ and $M_{2}=C_{7} x_{1} x_{2}$ or structure $\left(K_{12}\right)$. We used a linear pseudoinverse on the original dataset to calculate the coefficients $C_{0-7}$. The difference between parameters $K$ and $C$ was in the notation only; $C$ values were calculated numerically and used in the analysis of function and structure embedded in the polynomials (see below, Kinematic Muscle Invariants), and $K$ notation was used in the 
generation of polynomial structure (see next section). This algorithm embeds the constraint described in Equation 2-2 by expanding the selection of terms in the system of polynomials generated by the algorithm detailed next.

\section{Model generation and validation}

Muscle paths vary greatly in their complexity and, consequently, their model representations. The simplest muscles can be approximated with a constant if their path is posture independent, and complex muscles may involve many polynomial terms. The search for the optimal model requires the evaluation of each additional term from the domain of terms that grows exponentially with the number of actuated DOFs. Thus, muscles crossing 6 DOFs in our model were the most challenging. To solve this, we created an optimization algorithm based on the forward stepwise regression (see (Izenman, 2008), p. 142). This method was adapted to include the constraint in Equation 2-2 in the process of expanding the polynomial structure with additional terms until the information tradeoff indicated overfitting. For this purpose, we used the corrected Akaike Information Criterion (AICc) for a finite sample size (Akaike, 1974; Burnham and Anderson, 2004):

\section{Equation 2-3}

$$
\operatorname{AIC} c(f)=A I C(f)+\frac{2 k(k+1)}{N-k-1}=2 k-2 \ln (L)+\frac{2 k(k+1)}{N-k-1}
$$

where $f$ is an approximation function, $A I C$ is the Akaike Information Criterion, $k$ is the number of parameters in the model, $N$ is the number of data points, and $L$ is a maximum likelihood estimation (MLE) of the polynomial representing this dataset. The peak value of MLE for the normally distributed estimated residuals is $\ln (L)=-0.5 N\left(\ln \left(2 \pi \sigma^{2}\right)+1\right)=-N \ln (\sigma)+$ const, where $\sigma$ is the root mean square error. The model-independent constants are ignored in the substitution of $\ln (L)$ in Equation 2-3 because we use AICc values to compare multiple models (see further details on pp. 62-67 in Burnham and Anderson, 2004):

\section{Equation 2-4}

$$
\operatorname{AICc}(f)=2 k+2 N \ln (\sigma)+\frac{2 k(k+1)}{N-k-1}
$$


To remove potential differences between DOFs, we normalized the muscle length values to the range of motion and the moment arm values to their maximum across all physiological postures.

The analysis selected the terms of the polynomial structure for a muscle as follows (Figure 2-3A):

1. Initialize polynomials (without terms) for the functions approximating muscle length $L(x)$ and its set of moment arm functions, $\left\{M_{i}(x)\right\}$.

2. Make a list of potential candidates for the expansion of each polynomial using all possible combinations from the fifth-degree polynomial: $\Psi(L) ;\left\{\Psi\left(M_{i}\right)\right\}_{i}$.

3. Select optimal functions indicated by the smallest AICc values from the lists $\Psi(\bullet)$ and append them to the current approximation: $L(x)=\underset{f \in[\Psi(L) ; L]}{\operatorname{argmin}} A I C c(f), M_{i}(x)=$ $\underset{f \in\left[\Psi\left(M_{i}\right) ; M_{i}\right]}{\operatorname{argmin}} A I C c(f)$.

4. Use the algorithm, described above (Model Physical Constraints), to impose the relationship in Equation 2-2.

5. Return to step 2: i) if further expansion is possible $\left(\Psi(L)\right.$ or $\Psi\left(M_{i}\right)$ are not empty), and ii) the change in AICc values is negative between iterations.

The progression of model assembly with this algorithm can be seen in Figure 2-3B showing the optimization of kinematic variables for flexor pollicis longus with the iterative expansion. The first evaluation of errors was performed relative to zero model $\left(L(x)=0 ;\left\{M_{i}(x)\right\}=0\right)$. The errors for the selected terms were evaluated in the following iteration step. In the first iteration, the muscle length was approximated by $\left(a, K_{1}, K_{2}, K_{4}, K_{5}, K_{33}\right)$, where some terms came from the selection of terms in step 3 and the rest from the integration in step 4 . In the second iteration, the approximation expanded using elements $K_{11}, K_{44}, K_{55}, K_{333}, K_{2222}$, and the precision of muscle length fit decreases below 1\%. In the fifth iteration, only thumb CMC \& MCP moment arms required further optimization when other DOFs reached the minimum of AICC. In the tenth iteration, the evaluation of optimal parameter selection was finished with the high precision of $10^{-3}$ for the fit of muscle length across all physiological postures. Here, the worst moment arm fit of wrist extension-flexion (dashed blue line) was 1.05\% in units normalized to the range of motion and the maximum magnitude of moment arm or $0.2 \mathrm{~mm}$ in absolute units. 

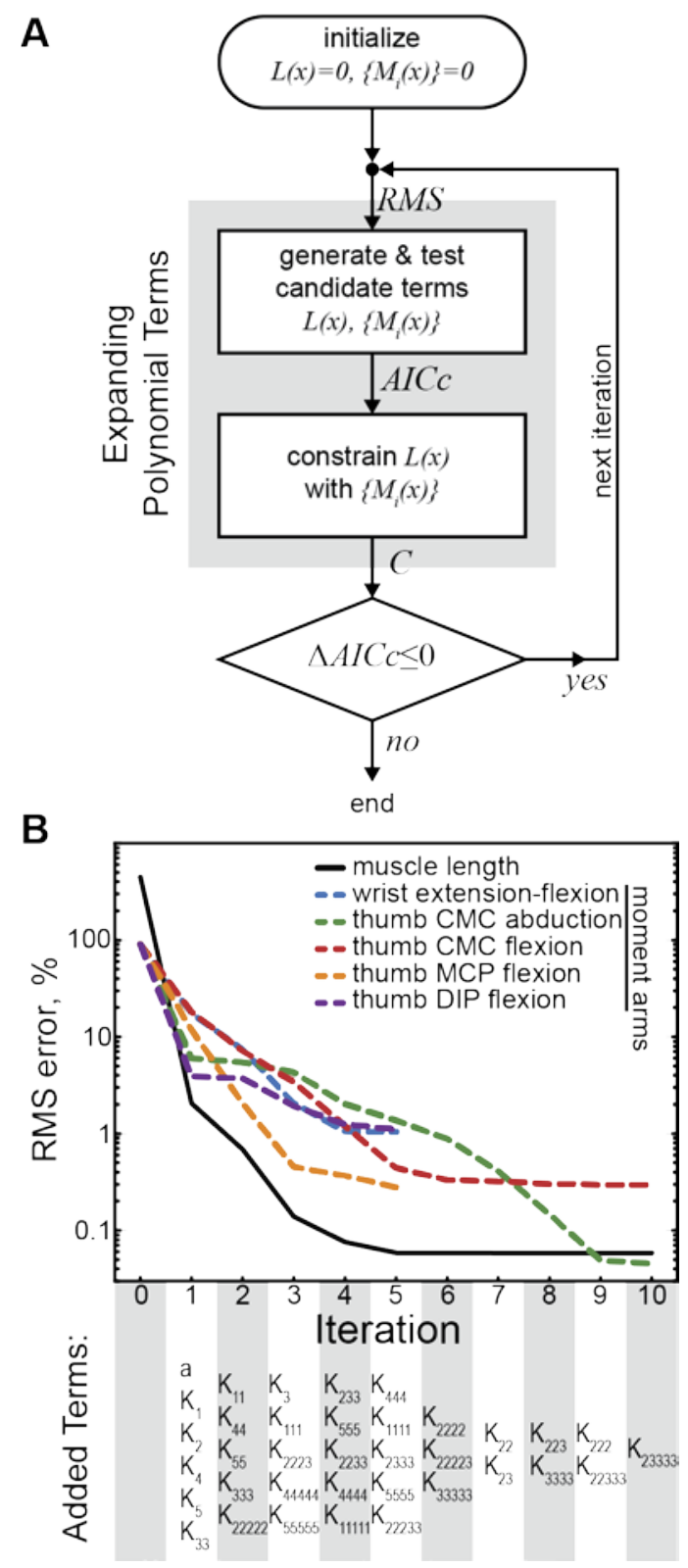

Figure 2-3. Optimal model generation algorithm.

A. The optimization flow schematic showing the flow of calculations using the amalgamated algorithm of model generation with physical constraint. RMS values of model performance are computed at the onset of each new iteration and followed by the expansion of polynomial candidates. The process continues while there are improvements in AICc metric. B. Example of generating the system of polynomial functions describing flexor pollicis longus. The decrease in RMS errors for all DOFs actuated by this muscle were plotted for each iteration of the algorithm. The progression of terms added to minimize AICC in 6 polynomials is shown below the plot. 


\section{Similarity index}

The polynomial approximations generated with and without adherence to Equation 2-2 were different. To measure that difference, we created a similarity index (SI) that counted common polynomial terms in two structures. Consider polynomials $L_{A}$ and $L_{B}$ characterizing muscles $\mathrm{A}$ and $\mathrm{B}$. Each polynomial can be described by a collection of shared or common terms $\left(P_{C}\right)$ and a collection of non-common terms $\left(P_{N C}\right)$, so that $L_{A}=P_{C} \cup P_{A N C}$ and $L_{B}=P_{C} \cup P_{B N C}$, where $P_{A N C}$ are the terms present in $L_{A}$ and not in $L_{B}$ and $P_{B N C}$ are the terms present in $L_{B}$ and not in $L_{A}$. Then, the similarity index is calculated as:

\section{Equation 2-5}

$$
\operatorname{SI}(A, B)=\frac{N_{C}}{N_{A N C}+N_{B N C}+N_{C}} \cdot 100 \%
$$

where $N_{C}, N_{A N C}, N_{B N C}$ are the number of terms in $P_{C}, P_{A N C}, P_{B N C}$, respectively. SI equals to $100 \%$ when two polynomials have completely identical structures, while the exact values of the coefficient at each polynomial term can be different; and to $0 \%$ when the structures completely different.

\section{Kinematic Muscle Invariant}

The composition of polynomials was captured using their representation in the Euclidean space formed by the basis of unique polynomial power terms. The expression of functional information embedded in the pattern of polynomials could then be tested by the examination of similarities between muscles in this space. For the full polynomial of power $\rho=5$ and maximum muscle dimensionality $d=6$ these unique combinations are the following: $[(1,1,1,1,1),(1,1$, 1, 1), (1, 1, 1, 2), (1, 1, 1), (1, 1, 2), (1, 1, 3), (1, 1), (1, 2, 2), (1, 2), (1, 3), (1, 4), (1), (2, 2), (2, 3), (2), (3), (4), (5)], where $(1,1,1,1,1)$ is, e.g., $x_{1} x_{2} x_{3} x_{4} x_{5}$ and (5) is $x_{i}^{5}$. The indices corresponding to which specific DOF is used in the equation are ignored (Table 2-1). The coefficients for these ordered 18 combinations defined the coordinates of a vector representing a given muscle length polynomial. We converted all polynomials into unit vectors with the normalized sums of coefficients of the same terms from different DOFs, $\hat{v}=\left(v_{1}, \ldots, v_{n}\right)^{T} /$ $\left\|\left(v_{1}, \ldots, v_{n}\right)^{T}\right\|$. 
Table 2-1. Examples of polynomial term notation and kinematic muscle invariants. $\left(x_{1}, x_{2}, x_{3}, x_{4}\right)$ are coordinates.

\begin{tabular}{|c|c|c|}
\hline Kinematic Muscle Invariant & Example polynomial term & Its structure \\
\hline (1) & $\begin{array}{l}x_{1} \\
x_{2}\end{array}$ & $\begin{array}{l}\mathrm{K}_{1} \\
\mathrm{~K}_{2}\end{array}$ \\
\hline$(2)$ & $x_{1}^{2}$ & $\mathrm{~K}_{11}$ \\
\hline (3) & $x_{1}^{3}$ & $\mathrm{~K}_{111}$ \\
\hline (4) & $x_{1}^{4}$ & $\mathrm{~K}_{1111}$ \\
\hline (5) & $x_{1}^{5}$ & $\mathrm{~K}_{11111}$ \\
\hline$(1,1)$ & $\begin{array}{l}x_{1} x_{2} \\
x_{2} x_{3}\end{array}$ & $\begin{array}{l}\mathrm{K}_{12} \\
\mathrm{~K}_{23}\end{array}$ \\
\hline$(1,2)$ & $\begin{array}{l}x_{1}^{2} x_{2} \\
x_{2} x_{3}^{2}\end{array}$ & $\begin{array}{l}\mathrm{K}_{112} \\
\mathrm{~K}_{233}\end{array}$ \\
\hline$(1,3)$ & $x_{1} x_{2}^{3}$ & $\mathrm{~K}_{1222}$ \\
\hline$(1,4)$ & $x_{1} x_{2}^{4}$ & $\mathrm{~K}_{12222}$ \\
\hline$(2,2)$ & $x_{1}^{2} x_{2}^{2}$ & $\mathrm{~K}_{1122}$ \\
\hline$(2,3)$ & $x_{1}^{2} x_{2}^{3}$ & $\mathrm{~K}_{11222}$ \\
\hline$(1,1,1)$ & $x_{1} x_{2} x_{3}$ & $\mathrm{~K}_{123}$ \\
\hline$(1,1,2)$ & $x_{1} x_{2} x_{3}^{2}$ & $\mathrm{~K}_{1233}$ \\
\hline$(1,1,3)$ & $x_{1} x_{2} x_{3}^{3}$ & $\mathrm{~K}_{12333}$ \\
\hline$(1,2,2)$ & $x_{1} x_{2}^{2} x_{3}^{2}$ & $\mathrm{~K}_{12233}$ \\
\hline$(1,1,1,1)$ & $x_{1} x_{2} x_{3} x_{4}$ & $\mathrm{~K}_{1234}$ \\
\hline$(1,1,1,2)$ & $x_{1} x_{2} x_{3} x_{4}^{2}$ & $\mathrm{~K}_{12344}$ \\
\hline$(1,1,1,1,1)$ & $x_{1} x_{2} x_{3} x_{4} x_{5}$ & $\mathrm{~K}_{12345}$ \\
\hline
\end{tabular}


For example, for $L=C_{1} x_{1} x_{2}^{2}+C_{2} x_{1}^{2} x_{2}+C_{3} x_{1}^{3}+C_{4} x_{1}+C_{5} x_{2}+C_{6}$, the vector has nonzero elements $\left[v_{9}=\left|C_{1}\right|+\left|C_{2}\right| ; v_{12}=\left|C_{4}\right|+\left|C_{5}\right| ; v_{16}=\left|C_{3}\right|\right]$. Structural difference of two polynomials can then be obtained as a distance between their vectors that we call muscle invariants. The structural difference is minimal when power composition of all terms and their absolute coefficients are similar in both polynomials even if they cross different DOFs, and large when their power compositions do not have the same terms.

\section{Memory and Time}

Memory required for spline approximation was calculated as a size of MATLAB's '.mat' files that contained single-precision spline parameters saved using '-v7.3' flag which enables compression. Memory required for polynomials was calculated as the size of executable '.mexw64' files compiled with Visual Studio 2017 C++ with '/O2' optimization. Time of evaluation was obtained using MATLAB's Profiler. Individual samples for mean and standard deviation of evaluation time were obtained per muscle's dataset during estimation of quality of fit. All computations were done on DELL Precision Workstation T5810 XL (Intel Xeon processor E52620 v3 2.4 GHz, 64 GB DDR4 RAM, SK Hynix SH920 512 GB SSD) running Windows 10.

\section{Statistics}

The validity of polynomials was analyzed with standard statistical tools. The root mean square error (RMSE) values were used to evaluate errors in the approximated values relative to the dataset used for fitting and the independent testing dataset (see above, Dataset). We detected outliers using a method similar to (Sartori et al., 2012), which resulted in the removal of less than $0.09 \%$ of values from the 9-point dataset. We estimated maximum expected error (MEE) using Chebyshev's theorem with 1\% significance level. Linear regression was used to test the relationship between the complexity of functions represented by the number of DOFs a muscle spans and the complexity of the approximating polynomials.

The similarity of muscle length invariants $(\hat{v})$ across multiple muscle groups was tested with dimensionality reduction analyses_-principle component analysis (PCA) and hierarchical clustering. The Euclidean distance between vectors was first analyzed with the average linkage hierarchical clustering implemented in SciPy. Then, the dominant relationships in this distribution of muscle invariant vectors were analyzed with PCA (Scikit-learn module (Pedregosa et al., 2011)). 
The representation of structural and functional information within the muscle length invariants was further tested by comparing the distributions of the distances between muscle pairs with similar structure or similar function to muscles with different structure or different function. These distributions were shown to be non-normal using D'Agostino's K-squared test (D'Agostino and Pearson, 1973) that measures deviation from the normal skewness and kurtosis. We used onetailed Mann-Whitney $U$ test ((Mann and Whitney, 1947), from SciPy module) to assess the two hypotheses that functional and structural similarities are represented in the colocalization of the invariant vectors. In general, this test was used to assess the likelihood of observing a smaller distance between the randomly selected pairs of muscle invariants with matching function or structure than the distance between the randomly selected pairs with shuffled function or structure. The smaller distances between the pairs in matched populations than the larger distances between the pairs from the shuffled populations were also tested with one-sided sign test (Conover, 1999). The symmetrical distribution of samples around the mean is not assumed in the sign test; thus, it is a better choice for this problem then Wilcoxon signed-rank test. All tests were performed with the conservative value of significance set at 0.01 .

\section{Results}

We developed a precise and efficient method to describe the musculoskeletal kinematics of a human distal arm and hand, extending previous work with approximation functions (Menegaldo et al., 2004; Sartori et al., 2012). Here, we formalized the dynamic selection of terms in a best-fit polynomial function using a quantitative tracking of overfitting; moreover, the structurally linked parameters of muscle length and moment arms were embedded in the derivation algorithm to generate consistent analytical models with the same linked properties. We tested if the composition of polynomials embedded information about muscle structure and/or function. 

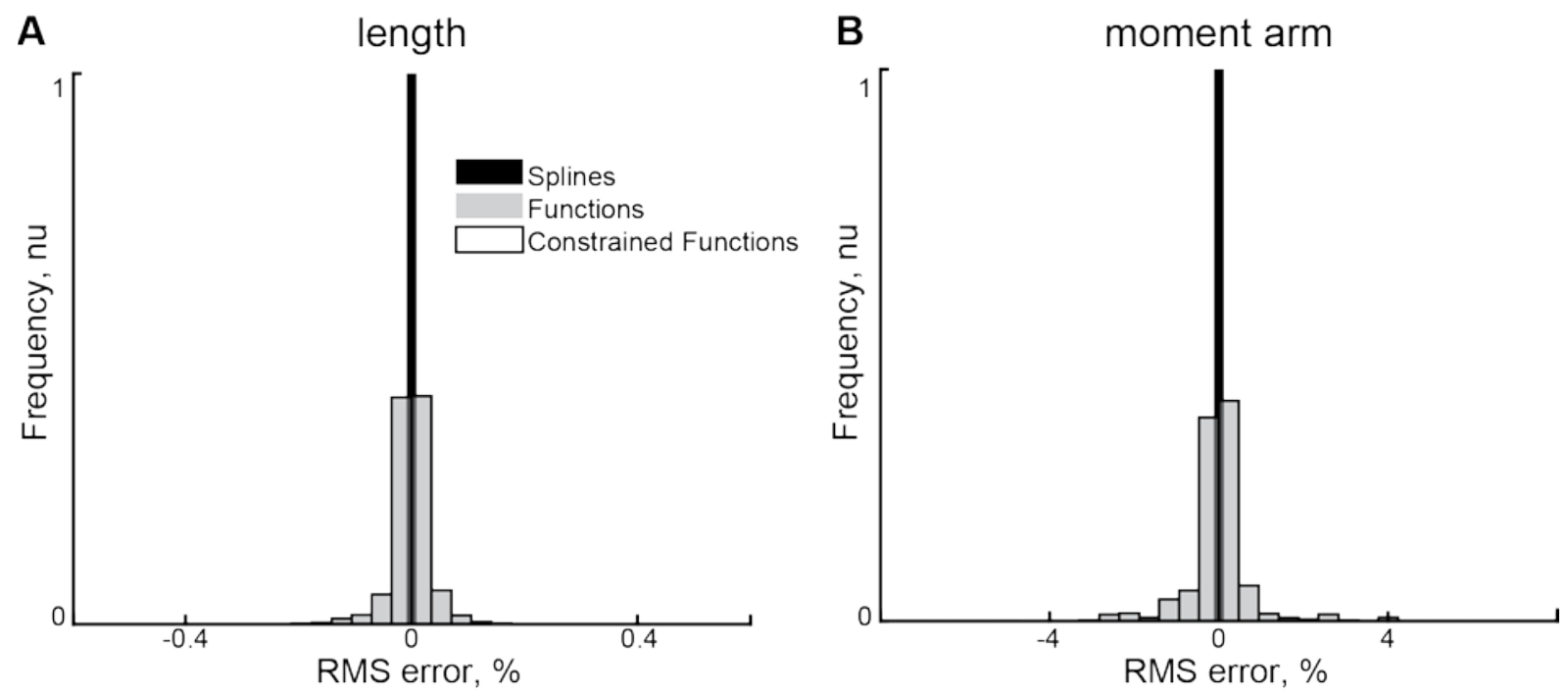

Figure 2-4. The goodness-of-fit test.

The distribution of normalized error in the estimation of muscle lengths $(\mathbf{A})$ and moment arms (B) is shown for three models (splines, polynomial functions with and without the constraint of Equation 2-2). The histogram frequency was normalized to the total count of samples.

\section{Approximation of muscle lengths and moment arms}

We subdivided values in the dataset (see above) into two groups for creating models and their testing. All best-fit models, splines and both types of polynomials, approximated moment arms with $<5 \%$ of error and muscle length with $<0.4 \%$ error, shown in Figure 2-4 and Table 2-1.

Although the approximation error with splines was the lowest, the implementation of splines required the highest number of parameters - eight orders of magnitude difference (compare cubic splines (CS) and constrained polynomials (CP) in Table 2-2). The large number of parameters in the CS model exceeded the number of values in the dataset, which corresponded to impractical AICc values. We used AIC values instead to compare the relative quality of models: CP values were $-6.7^{*} 10^{6}$ and $-5.7 * 10^{5}$, as compared to CS values were $2.2 * 10^{9}$ and $3.2^{\star} 10^{10}$. This difference indicates the preference of AIC metric to CP model. The addition of model physical constraints (Equation 2-2) to the polynomial generation algorithm did not significantly change the precision of the polynomial model $(p>0.9)$ with similar errors and AIC values in Table 2-2. The histograms of error distributions were superimposed in Figure 2-4. The 
length approximation errors in Figure 2-4A were smaller than those of moment arm errors in Figure 2-4B, as expected from Equation 2-2. In general, the differentiation process increases the magnitudes of errors.

Figure 2-4 and Figure 2-5A show the comparison of polynomial structures with and without the physical constraint imposed by Equation 2-2 in step 4 of the algorithm described in Methods: Model Generation and Validation. Because the constrained muscle length function has higher polynomial power than its moment arm functions, we used $\rho=4$ to generate $\Psi\left(M_{i}\right)$, and $\rho=5$ to generate $\Psi(L)$.

A small portion of values in the datasets were marked as outliers and removed from further analyses: unconstrained polynomials had $0.08 \%$ muscle length outliers and $0.03 \%$ moment arm outliers; constrained polynomials had $0.08 \%$ and $0.03 \%$, respectively. No spline errors were considered as outliers.

Table 2-2. The comparison of model performance.

Cubic spline (CS) and two polynomial approximations with and without the constraint linking muscle lengths and moment arms (constrained and unconstrained polynomials, CP and UP), as described by algorithm in Model Physical Constraints in Methods. RMSE values are given \pm standard deviation.

\begin{tabular}{|c|c|c|c|c|c|c|}
\hline \multirow[t]{2}{*}{ Method } & \multicolumn{2}{|l|}{ RMSE, \% } & \multicolumn{2}{|c|}{$\begin{array}{l}\text { Total number of } \\
\text { parameters }\end{array}$} & \multicolumn{2}{|l|}{ AIC, au } \\
\hline & L & MA & $L$ & MA & L & MA \\
\hline CS & $1.34 * 10^{-5} \pm 1.56 * 10^{-5}$ & $1.84^{\star} 10^{-6} \pm 2.47^{\star} 10^{-6}$ & $1.1 * 10^{9}$ & $1.64 * 10^{10}$ & $2.2 * 10^{9}$ & $3.2 * 10^{10}$ \\
\hline UP & $0.0383 \pm 0.0918$ & $0.757 \pm 1.477$ & 610 & 705 & $-6.7 * 10^{6}$ & $-5.7 * 10^{5}$ \\
\hline $\mathrm{CP}$ & $0.0382 \pm 0.0910$ & $0.757 \pm 1.477$ & 661 & 783 & $-6.7 * 10^{6}$ & $-5.7 * 10^{5}$ \\
\hline
\end{tabular}

The evaluation time of models was obtained by measuring the period of approximating full musculotendon dataset $(\mathrm{N}=33)$. Both polynomial models were over 7000 times faster than $\mathrm{CS}$ (Table $2-3$ ) and required $2.8 * 10^{5}$ times less memory. The search time for CP was 3.3 times 
faster than that for UP with the increase in performance gained when the selection of polynomial terms originated in the relationship between muscle length and moment arms.

Table 2-3. Time and memory requirements of approximations methods for kinematic variables.

\begin{tabular}{l|l|l|l}
\hline Method & Evaluation, $\mu \mathrm{S}$ & Generation, min & Memory, KB \\
\hline CS & $7.8^{*} 10^{4} \pm 0.7 * 10^{4}$ & 32 & $20.6 * 10^{6}$ \\
UP & $9.7 \pm 2.9$ & 243 & 69 \\
CP & $9.9 \pm 2.0$ & 74 & 73 \\
\hline
\end{tabular}

\section{Structure of Approximating Polynomials}

Both CP and UP models were similar in composition as determined by the similarity index. It examined the difference in polynomial structure, i.e. the presence or absence of terms in functions for muscle lengths. Figure 2-5A shows that both models had the maximum difference of $40 \%$ in the similarity index (BIC_SH: 40.0, FCU: 36.8, ADPT: 30.4), and the average difference was only $12.91 \%$. This indicates that composition of CP and UP models were similar, which in turn supported the validity of generated polynomials.

We expected to see an exponential relationship between muscle model complexity (i.e., the number of parameters) and the number of DOFs crossed. The relationship shown in Figure 2-5B is instead linear $(r=0.74)$. Moreover, the model fractional complexity, measured as the ratio of terms selected to all possible terms available, decreased with the increase of the number of DOFs controlled by a muscle (Figure 2-8, $r=-0.83$ ). The most complex muscles in our model were thumb muscles (ADPT, FPB, APB, EPB, APL, FPL, EPL), and they appeared above the regression line, while finger muscles (FDS2-5, FDP2-5, ED2-5, EDM, EIND) stayed below, suggesting a lower relative complexity. 


\section{A}

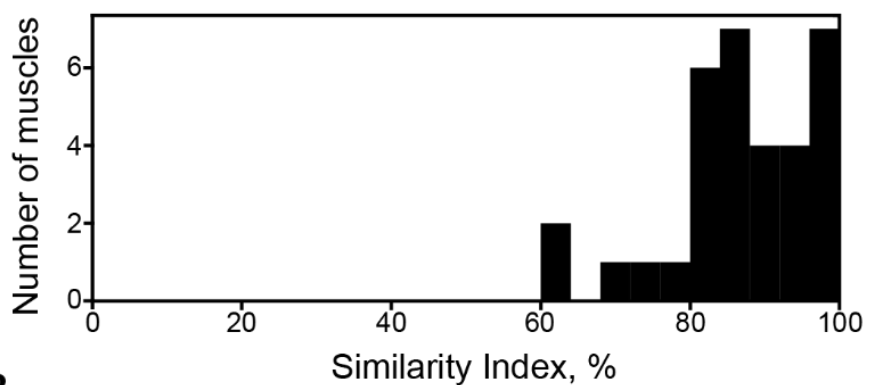

B

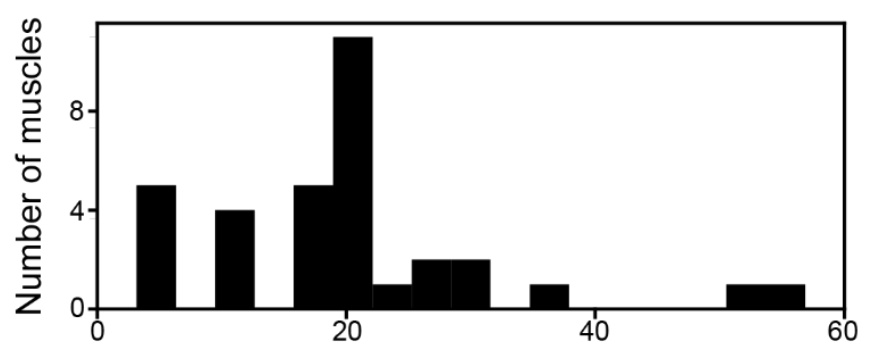

C

Number of terms

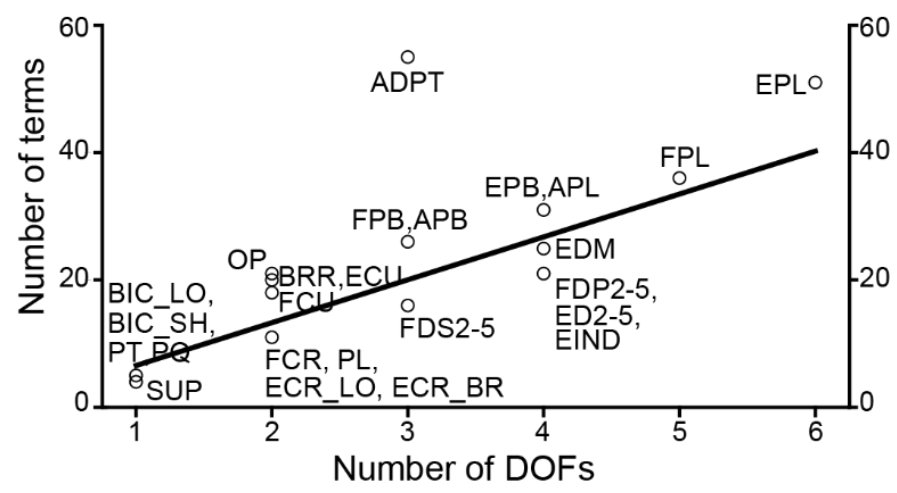

Figure 2-5. Complexity of muscle structures.

A. Similarity index between functions approximating muscle lengths generated with and without adherence to Equation 2-1. B. The distribution of polynomial complexity expressed as the number of terms. $\mathbf{C}$. The relationship between the number of terms in the muscle length polynomial (circles) and the number of DOFs the muscle spans (line, $y=6.73 x-0.16, r=0.74$, $\left.p<2 \cdot 10^{-6}\right)$. 


\section{Structure and Function}

We hypothesized that the generated models capture structural and functional features of muscles and developed a measure of embedded muscle attributes, coined muscle invariant. It represents each muscle in the space of polynomial term powers. To avoid trivial relationships, we remove DOF identity information and preserve only the power signature of each term (see details in Methods).

The difference between muscles was captured as Euclidean distances between their vectors. To visualize the 18-dimensional space of all power terms, the distance heatmap was calculated between all muscle pairs (Figure 2-6A), and the corresponding vectors were plotted in the axes of two main principle components computed with PCA (Figure 2-6B). The clustering algorithm generated the average-link dendrogram based on these distances. A selection of distal thumb muscles (ADPT, APB, OP, APL) was visibly separated from about 6 other subgroups, with the closest subgroup formed by another subset of thumb muscles (EPL and EPB). The thumb muscles are followed by the following subgroups: extensor carpi radialis and wrist flexors (ECR_LO, ECR_BR, FCR, PL), flexor pollicis brevis and extensor carpi ulnaris (FPB and ECU), finger and wrist flexors and extensors, wrist rotators located in the forearm (FDP2-4, FDS3-5, ED2, ED4, ED5, EIND, PL, FCR, PQ, PT, SUP), the rest of digit muscles with flexor carpi ulnaris (ED3, EDM, FDS2, FDP5, FCU, FPL), and biceps brachii (BIC_SH, BIC_LO).

The variance of muscle invariants was largely captured by the first two principal components (86\%). Their largest coefficients were associated with linear $\left(\hat{v}_{\{x\}}=-0.68\right)$ and square $\left(\hat{v}_{\left\{x^{2}\right\}}=\right.$ $0.84)$ powers of polynomial terms. The linear relationship between joint angle and muscle length corresponds to a semi-circle muscle path around a joint. This simplistic behavior is characteristic for 1-DOF finger joints, muscles in the bottom-left corner and the insert of Figure 2-6B. Muscles in the bottom-right corner, e.g., thumb muscles, used less linear terms than other muscles. Overall, the space of muscle invariants has a nonrandom pattern. 
A

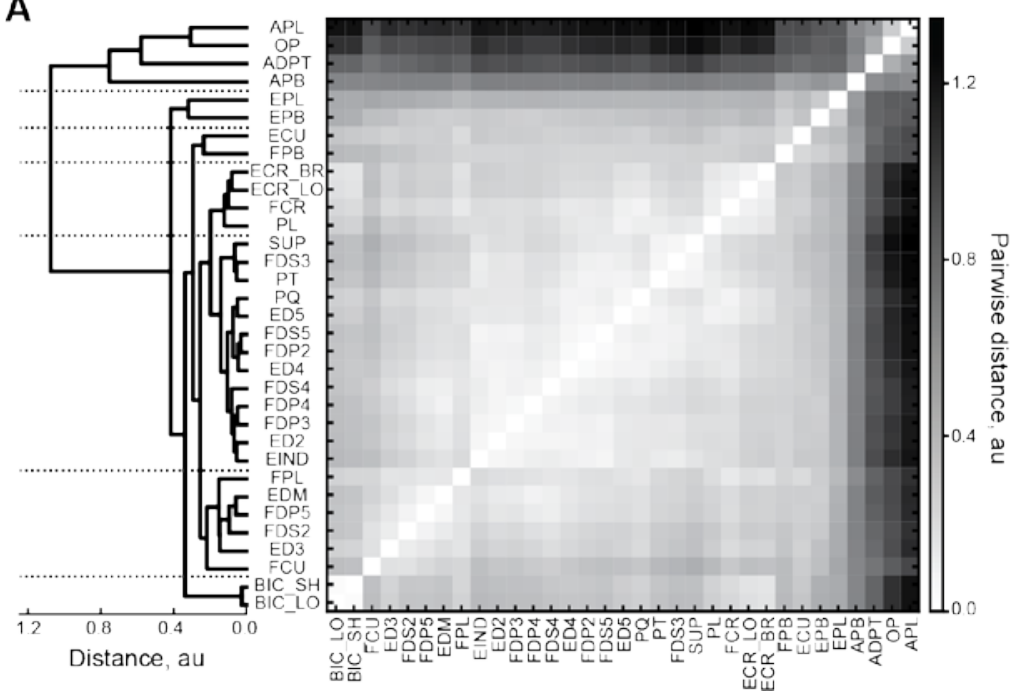

B

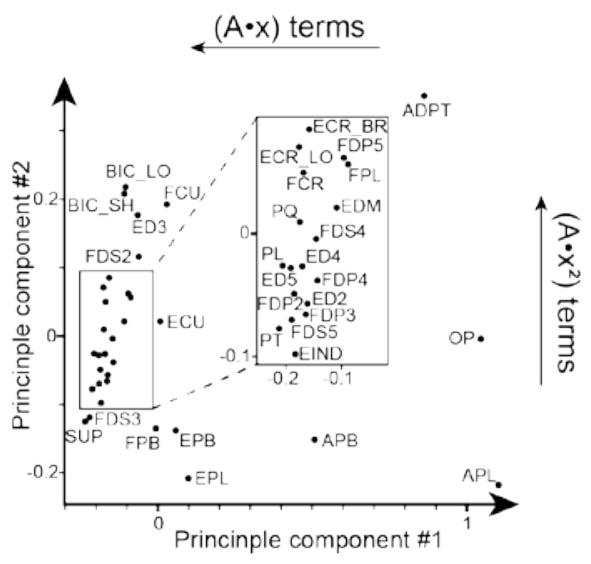

Figure 2-6. Kinematic muscle invariants.

A. Average-linkage dendrogram computed from the heatmap of pairwise distances between muscle invariants. Horizontal dashed lines indicate subgroups described in text. B. The representation of muscle invariants in the space of their main two principle components. Insert: expanded view of a portion of the plot.

We tested if muscle invariants contain information about their anatomical location by comparing Euclidian distances between the invariants with shared DOFs. Since there is a limited set of muscles that do not span the same joints, we tested the idea that those pairs of muscles that share a given DOF would be closer to each other than those that do not share that DOF. We assigned phalangeal DOFs (MCP, PIP, DIP) to be different to each other, but the same across fingers $2-5$ because of their similarity and the lack of intrinsic hand muscles (e.g., lumbricals) in the model. This selection ensured the local structural similarity in the group with a shared DOF (Figure 2-7A, blue) and local difference in the group without a shared DOF (Figure 2-7A, red), but it did not prevent the selection of muscle pairs in each group based on their structure relative to other DOFs. Figure 2-7A shows the probability of observing a given distance between a pair of muscles with a shared DOF and without a shared DOF based on 1306 and 1862 pairs, respectively. The selection of muscles into these groups was executed sequentially by examining all muscles for each DOF in the model. The difference distribution (Figure 2-7C) was computed by examining the difference between each pair with a shared DOF 
and comparing it with each pair that had one of the two muscles in the group without a shared DOF, resulting in 20,746 comparisons.

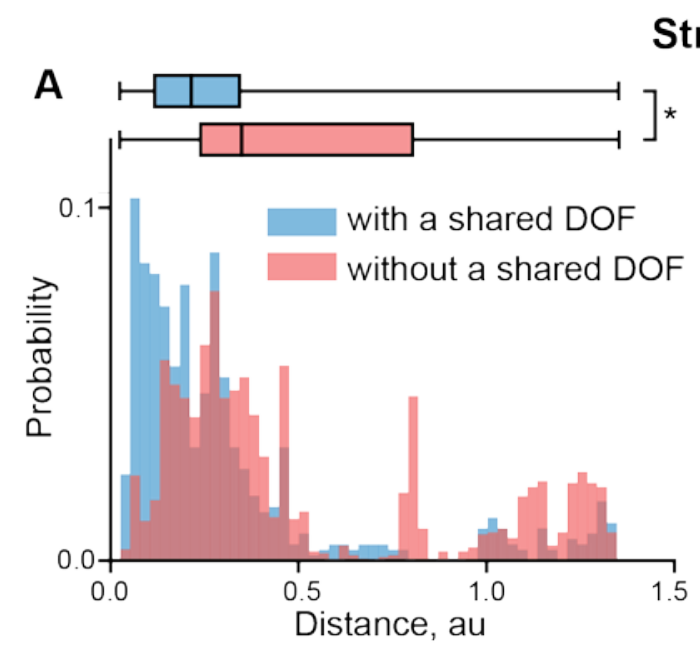

Structure

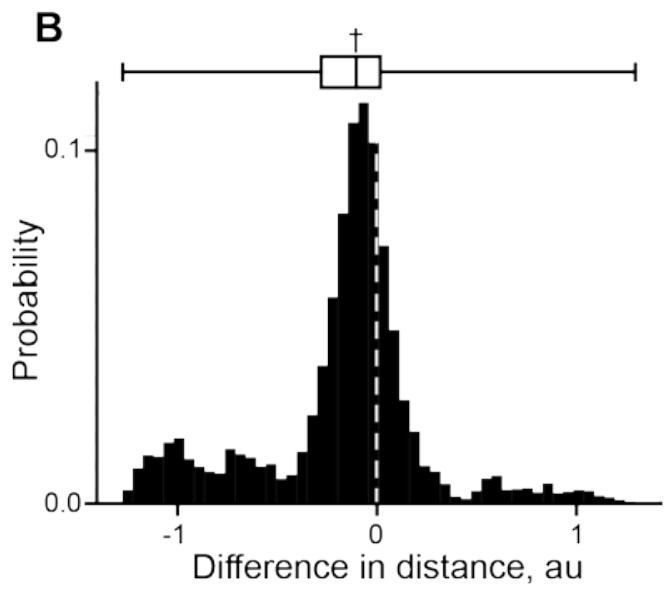

Function
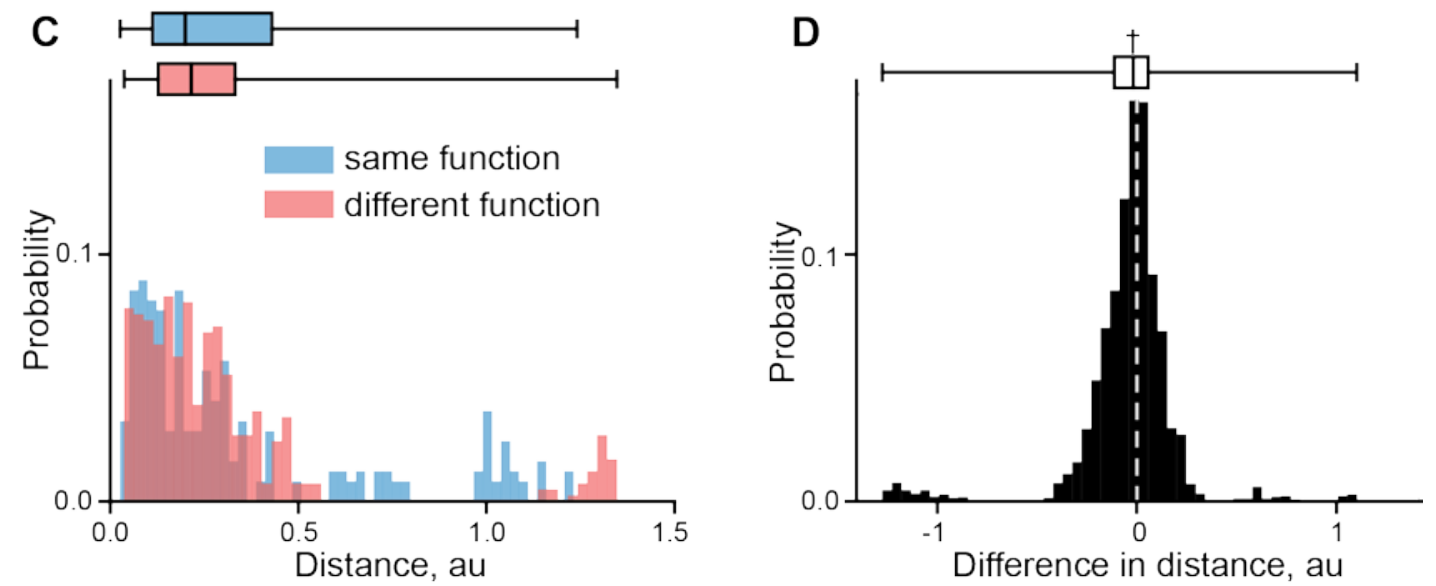

Figure 2-7. The structural and functional information embedded in muscle invariants.

A. The probability distributions of observing the distance between the pairs of muscle invariants with (blue) and without (red) a shared DOF. B. The test of difference between the two groups. C. The probability distributions of the distance between the pairs with the shared structural information and with (blue) and without (red) shared functions. D. The test of difference between the two groups. Box plots indicate a median and $25^{\text {th }}-75^{\text {th }}$ quantile region. The significant differences between the overlap of distributions tested with Mann-Whitney $U$ test is marked with $\left(^{*}\right)$. The sign test significance is marked with $(\dagger)$. 
The median of difference was less than zero $\left(-0.10\right.$, sign test $\left.p<10^{-8}\right)$. Both groups were not normally distributed (D'Agostino's K-squared test of normality, $p<10^{-8}$ ) and similar anatomical pairs were closer to each other which was evident from the non-equal distribution of the two groups (Mann-Whitney $U$ test: $U=7 \cdot 10^{5}, p<10^{-8}$ ). We found that the muscle invariants capture the structural information related to the identity of their actuated DOFs.

We tested if the muscle invariants contain functional information beyond that explained by the anatomical similarities. For this purpose, we defined seven functional groups based on their primary mechanical function: wrist supinators (BIC_LO, BIC_SH, SUP), pronators (PT, PQ), extensors (ECR_LO, ECR_BR, ECU), flexors (FCR, FCU, PL), finger flexors (FDS2-5, FDP2-5), extensors (ED2-5, EDM, EIND), and thumb muscles (APL, OP, APB, EPL, EPB, FPB, FPL, ADPT). We tested the idea that two muscles from the same group are closer together than those from different groups even when all these muscles actuate the same DOF. Similar to above, we selected all pairs of muscles with (490 pairs) and without (816 pairs) a shared function and computed the distance between these pairs, shown in in Figure 2-7C. The distance between the two groups based on the combinations of all these pairs (3496 samples) is shown in Figure 2-7D. These three distributions were also not normal $\left(p<10^{-8}\right)$. While the distributions of two groups were overlapping $(p=0.61)$, the median of difference between them was significantly less than zero $\left(-0.02\right.$, sign test $\left.p<10^{-8}\right)$. This supports the hypothesis that DOF-independent functional differences are captured by the muscle invariants.

\section{Discussion}

We approximated musculoskeletal kinematics of the human hand with a new type of autogenerating model that embeds biomechanical constraints between muscle parameters. The model reached optimal performance with polynomial simulations showing high precision and computational efficiency. While the model was developed as a descriptive tool, the fine details captured within the muscle-posture relationships include the differential connection between moment arms and muscle lengths and reflect the high-level mechanistic properties of arm and hand muscle function. The composition of terms in these models was objectively determined by the embedded information and demonstrated the patterns associated with anatomy and function. The mechanical specification of muscles for the control of different hand DOFs and different functions has not been previously demonstrated, and the implications are discussed. 
All models are simplifications or approximations of reality, but some approximations are useful. The complex geometric interactions_sliding and wrapping—between muscles and other mechanical body structures pose a considerable computational challenge for real-time applications (Blana et al., 2017). The engineering trade-off between complexity, performance, and accuracy pushed the development of simplified biomechanical limb models that assumed constant moment arm and posture relationships (Crouch and Huang, 2016) or reduced the span of musculotendon anatomy to ease the computational demand (Durandau et al., 2018). The approximating models can be mechanistic or phenomenological. The goal of phenomenological model is to capture the input-output relationship without the effort of describing the mechanistic explanation present within this transformation. We replaced the customary subjective choice of structure in approximations with our objective method of generating the approximations that may reveal mechanistic aspects of MS organization in the phenomenological model.

\section{Autogenerating models}

Interest in MS approximations has been steadily increasing with the development of computational tools for human motion analysis, e.g., OpenSim (Delp et al., 2007). Accuracy of these approximations has been demonstrated with B-spline models (Sartori et al., 2012; Durandau et al., 2018), and computational efficiency has been achieved with polynomial models (Menegaldo et al., 2004; Chadwick et al., 2009). The optimal polynomials proposed in this manuscript have the benefits of both accuracy and computational efficiency.

The manual subjective selection of polynomial terms for each muscle is usually based on the number of DOFs the muscle crosses, the quality of simulation, and the numerical cost of evaluating functions. In contrast, our optimization algorithm chooses the polynomial terms objectively based on the information criterion to reflect objective dependencies within the data. The information criterion is a type of cost function that allows comparison between different polynomial models and prevents overfitting with an excessive number of terms. The latter is possible when using the subjective desired precision of fit, as in (Chadwick et al., 2009). Similar to (Menegaldo et al., 2004), the number of terms in the optimized polynomial grows with the number of muscle's DOFs, but the term composition varies to reflect the diverse anatomy and function.

We found multiple levels of structure embedded in the power composition of polynomial terms. A linear relationship between muscle length and joint angle is characteristic for 1-DOF 
finger joints. The near-linear relationship between moment arm profile and joint angle we showed in thumb muscles has been commonly observed in other studies (Loren et al., 1996; Menegaldo et al., 2004). The physiological function of this relationship can be associated with compensation for the muscle force-length relationship at the edges of range of motion. The diverse function and behavior of thumb muscles found during movement (Kaufman et al., 1999) is mirrored in our results by their separation from other muscles and high variability between each other.

Previously we have examined the grouping of muscles based on their length-posture relationships where the similarity between muscles was determined by common muscle length shortening and lengthening in response to postural changes (see Fig. 7 in Gritsenko et al., 2016). The current analysis of muscle organization does not separate antagonistic muscles, with the focus only on the polynomial sets that shape muscle paths. Similar to the previous analysis, thumb muscles are clearly separated from other finger muscles. We have also included muscles with antagonistic functions in separate groups in the analysis of muscle properties captured by the model (Figure 2-7CD). This test indicated a functional difference between the muscle invariants even when the differences accounted by muscle location were removed; albeit, the difference was small. The analysis without removing the muscle location (using Similarity Index) yielded expected, but uninteresting results (Figure 2-9). This result supports the idea that the commonly observed muscle synergies during movement can be at least in part explained by the structure and function embedded in their musculotendon paths.

\section{Real-time high-dimensional musculoskeletal computations}

The optimal polynomials efficiently compute highly complex MS kinematics for real-time applications. The polynomials describing 33 musculotendon actuators each crossing up to 6 DOFs can be evaluated within $10 \mu \mathrm{s}$, requiring less than $75 \mathrm{~KB}$ of RAM. To contrast, the previous state-of-the-art performance for a lower-limb model with 13 musculotendon actuators, each crossing up to 3 DOF was shown to be less than $2.5 \mathrm{~ms}$ (Durandau et al., 2018). Our more than hundred-fold time efficiency improvement on the method was also accompanied by a similar improvement in required memory (about 10MB worth of coefficients in Durandau et al., 2018, based on Sartori et al., 2012). The improvements are largely due to the exponential rise in the required computational resources with the dimensionality increase of the spline model, as previously shown (Sartori et al., 2012) and by our implementation. This 'dimensionality curse' may prevent the application of splines in complex models recently developed for offline 
analyses (Holzbaur et al., 2005; Paclet and Quaine, 2012; Rajagopal et al., 2016). Our optimal polynomial approach shows linear scaling of the model (Figure 2-5C) allowing these models to be used in real-time applications.

The described optimization algorithm is structurally similar to stepwise regression (Izenman, 2008), but has several important differences. First, it automatically constructs and explores all possible polynomial combinations of the input variables within reasonable power limitations. Second, our algorithm uses AIC (Akaike, 1974; Burnham and Anderson, 2004) instead of Fstatistic as the objective measure of improvements. The AIC takes into account the trade-off between the quality of fit and the increased model complexity. This is a novel use of information measures (Akaike, Bayesian and other) that have been previously used mostly as a stopping criterion (Bendel and Afifi, 1977). An information criterion allows flexibility when choosing the tradeoff between quality of fit and the measure of model complexity. For example, using the number of processor commands instead of the number of variables for each term is useful for development of extremely high-performing routines or for computationally-costly devices, like portable chips or GPUs. Third, our approximation algorithm embeds the differential relationship between muscle length and its moment arms in the search for the best model parameters. This novel approach of using the formulation of structural constraints within the algorithm decreased model assembly time. These approximations are ready to be used on a portable device that requires a real-time simulation of MS variables, e.g., a biomimetic prosthesis or a medical assessment device.

\section{Limitations}

We chose to implement the fitting algorithm with the use of polynomial sequences as the most accurate representation of the MS relationships. The alternative implementations could use sequences of trigonometric or exponential terms. For example, any data with periodic relationships would be efficiently represented by trigonometric functions, and any data with sigmoidal transitions or limits of range could be represented by exponential functions. However, the relationships between moment arms and posture are smooth because of soft tissue properties. In this case, we can rely on the theoretical conclusion from Taylor's Theorem stating that any smooth function can be described with a polynomial approximation. Then the only potential failures would be the observations of discontinuities in the muscle properties. We have indeed observed sharp transitions always associated with the geometric model failures where muscle path slipped off the wrapping surface. These behaviors were detected and corrected 
prior to the approximation (Boots et al., 2019). Thus, our model is appropriate for the physical system it attempts to represent.

The autogenerating polynomial models were iteratively created with the selection of a single term per equation at a time. This allowed fast optimization for the full system of equations describing moment arms and muscle lengths. It is possible that multiple terms can be more optimal than a single term. This would be indicated by the premature termination of the optimization routine even when a more optimal solution is available for multiple terms selected in the same iteration. We tested this eventuality by repeating the model generation with an algorithm capable of adding one or two terms per iteration per equation. This method produced the same solutions for our dataset, but the evaluation time increased by an order magnitude as compared to the standard method.

The sampling rate of the relationship between posture and muscle parameters was another methodological implementation decision. The validity of our selection was tested by comparing the quality of approximation with three different rates, i.e., the training datasets were sampled at 3,5 , and 9 values per DOF. The corresponding three testing datasets with data points residing between the training data points were used for validation. The overall fitting errors were not significantly different between 5- and 9-point datasets. However, infrequent failures in the 5point model were effectively resolved with the 9-point model. Our observations suggested that further increases in the sampling rate is not likely to increase the model performance and may lead to the overfitting by exceeding the quality of the MS representation in OpenSim. Since the 5-point model had a very similar performance to the 9-point model, it can be effectively used as an intermediate fast approximation for iterative adjustments needed to validate muscle geometry against experimental data, as in (Boots et al., 2019). Overall, the 9-point model was deemed to be optimal.

The current model is limited to the description of hand muscles in a generic representation of the human hand. Future analysis of validated models that span the shoulder will improve our understanding of muscle specialization. We expect to see new functional groups with the structure different from that of any of the hand functional groups because of the unique biomechanics of the shoulder joint (Donald, 1973; Voisin, 2006). These functional groups can be then further refined by their evaluation on models with subject-specific segment scaling and morphometric differences (Akita and Nimura, 2016a). It will be also intriguing to compare the muscle organization of the upper limb to that of the lower limb, considering their proposed 
coevolution (Rolian et al., 2010), covariability in developmental modules (Hallgrímsson et al., 2002) and high observed topological similarity (Diogo et al., 2013) in humans. However, accurate and valid lower-limb models are still under development. Our future directions of research include the expansion of the model to the other joints and subject-specific modelling.

\section{Conclusions}

We approximated the kinematic variables for human hand and forearm muscles with high precision ( $<5 \%$ error across 18 DOFs) and efficiency ( $<75 \mathrm{~KB},<10 \mu \mathrm{s}$ ). The approximation algorithm utilized the relationship between moment arms and muscle lengths to generate approximation faster and with internal consistency. The approach overcomes the curse of dimensionality with increased complexity for large MS models. The structural content of optimal polynomials reflects muscle anatomy and function. This novel description can be further applied in neuromechanics and its applications.

\section{Supplementary information}

A

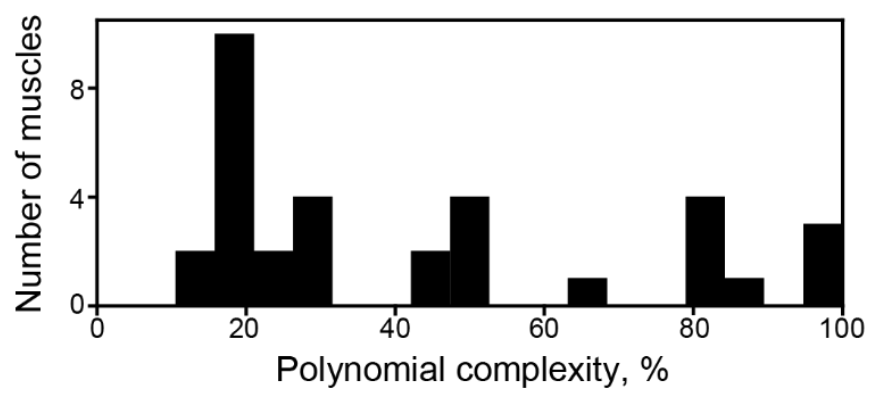

B

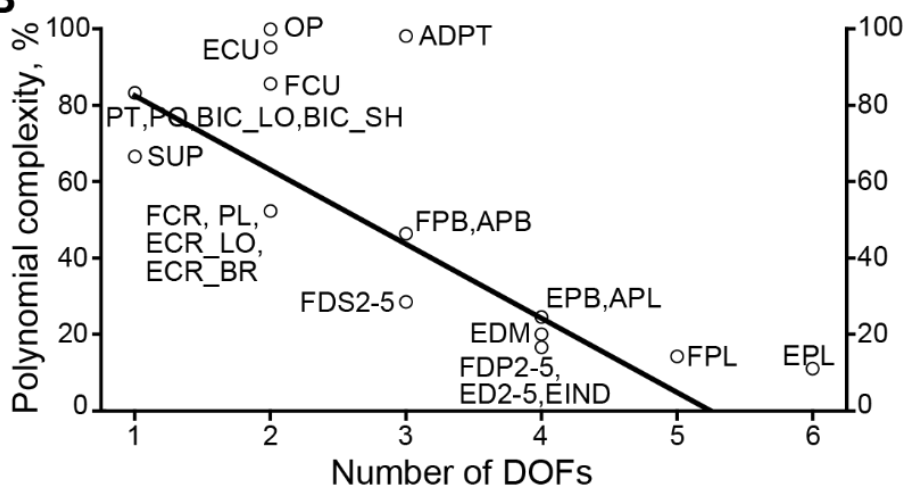

Figure 2-8. Complexity of muscle structures. 
A. The distribution of relative polynomial complexity expressed as the portion of parameter space used. B. The relationship between the relative complexity of the muscle length polynomial (circles) and the number of DOFs the muscle spans (line, $y=-19.4 x+101.9, r=$ $\left.-0.83, p<3 \cdot 10^{-9}\right)$. Relative complexity of a polynomial was estimated as a fraction of the parameter space that the polynomial occupies. For example, if the number of terms in the 2dimensional polynomial is 3 , and the size of the parameter space of 2-dimensional polynomial of power 2 is 6 , and the relative complexity is $\frac{3}{6}=0.5=50 \%$.

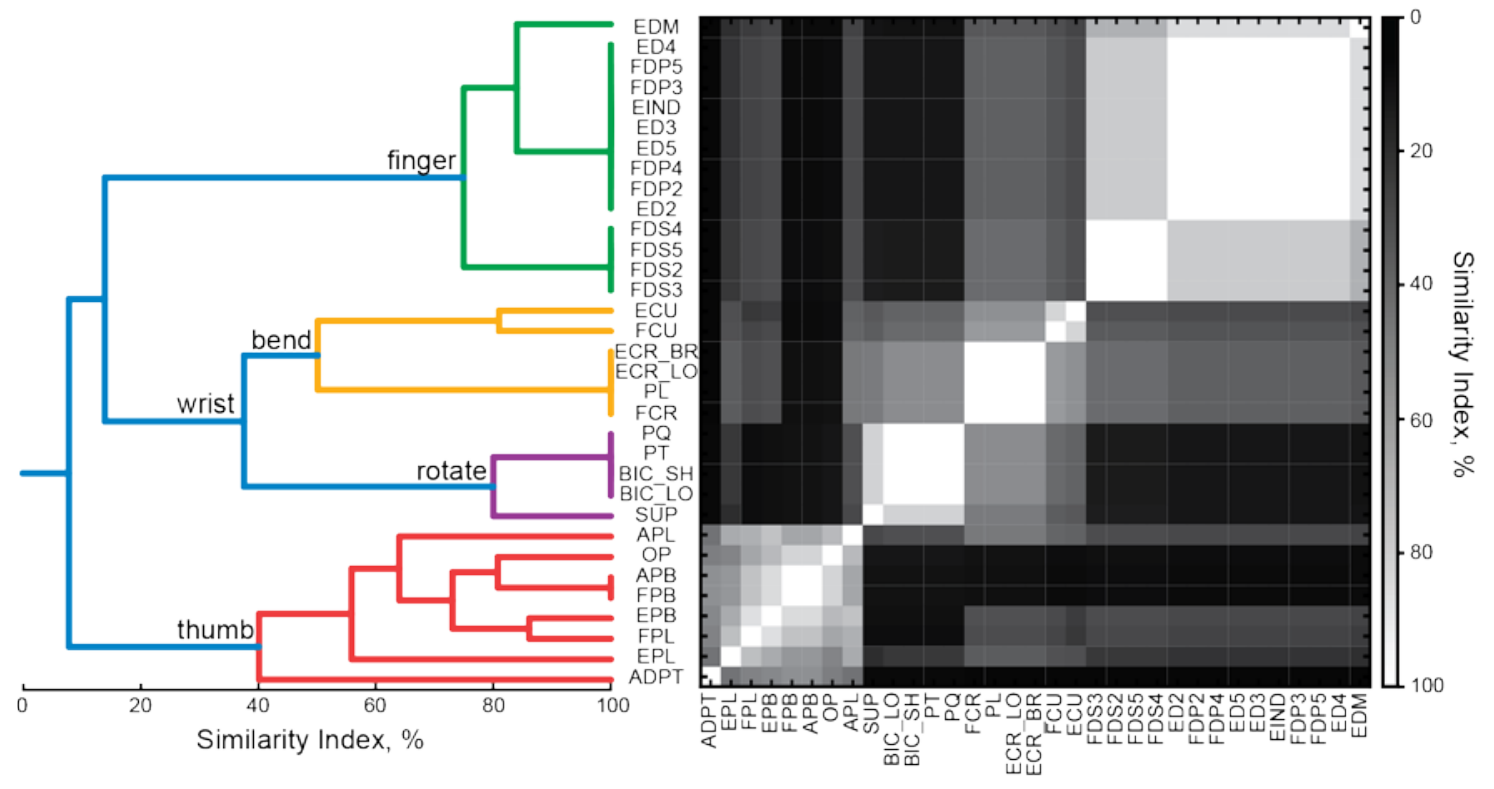

Figure 2-9. Similarity of muscle structures using Similarity Index.

Average-linkage dendrogram computed from the heatmap of pairwise Similarity Index. The distance between clusters was calculated as an average distance between elements of two clusters.

Table 2-4. The list of simulated DOFs.

Name of the DOF is the unique name used in simulations and figures. It has the following

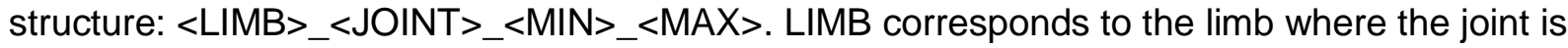


located, with 'ra' meaning 'right arm'. JOINT is the joint at which the DOF is located, for example 'wr' for 'wrist'. Digit joints have a number at the end identifying the identifying number of the finger: 1: thumb; 2: index; 3: middle; 4: ring; 5: pinky. The last two suffixes MIN and MAX indicate the anatomical position of the DOF at the minimum and maximum of the range, e.g., "ra_wr_s_p" indicates that the minimum of the wrist pronation-supination DOF at -1.5708 is the supinated posture, and the maximum, 1.5708 - pronated.

\begin{tabular}{|c|c|c|c|}
\hline id & Name & Range, rad & Description \\
\hline 1 & ra_wr_s_p & -1.57081 .5708 & wrist pronation/supination motion \\
\hline 2 & ra_wr_e_f & -1.22171 .2217 & wrist flexion/extension motion \\
\hline 3 & ra_cmc1_f_e & 00.8727 & thumb proximal flexion/extension motion \\
\hline 4 & ra_cmc1_ad_ab & 00.8727 & thumb proximal abduction/adduction motion \\
\hline 5 & ra_mcp1_f_e & -0.78540 & thumb central flexion/extension motion \\
\hline 6 & ra_ip1_f_e & -1.57080 & thumb distal flexion/extension motion \\
\hline 7 & ra_mcp2_e_f & 01.5708 & index proximal flexion/extension motion \\
\hline 8 & ra_pip2_e_f & 02.0944 & index central flexion/extension motion \\
\hline 9 & ra_dip2_e_f & 01.5708 & index distal flexion/extension motion \\
\hline 10 & ra_mcp3_e_f & 01.5708 & middle proximal flexion/extension motion \\
\hline 11 & ra_pip3_e_f & 02.0944 & middle central flexion/extension motion \\
\hline 12 & ra_dip3_e_f & 01.5708 & middle distal flexion/extension motion \\
\hline 13 & ra_mcp4_e_f & 01.5708 & ring proximal flexion/extension motion \\
\hline 14 & ra_pip4_e_f & 02.0944 & ring central flexion/extension motion \\
\hline 15 & ra_dip4_e_f & 01.5708 & ring distal flexion/extension motion \\
\hline 16 & ra_mcp5_e_f & 01.5708 & pinky proximal flexion/extension motion \\
\hline 17 & ra_pip5_e_f & 02.0944 & pinky central flexion/extension motion \\
\hline 18 & ra_dip5_e_f & 01.5708 & pinky distal flexion/extension motion \\
\hline
\end{tabular}




\section{Table 2-5. The list of simulated muscles.}

Name column describes the short name used in simulations and figures. Full name describes the anatomical name of the muscle and specifies the head of the actuator. DOFs lists all DOFs that each muscle spans by referencing ids from the Table 2-4.

\begin{tabular}{|c|c|c|c|}
\hline id & Name & Full name & DOFs \\
\hline 1 & BIC_LO & Biceps brachii long head & 1 \\
\hline 2 & BIC_SH & Biceps brachii short head & 1 \\
\hline 3 & SUP & Supinator & 1 \\
\hline 4 & PT & Pronator teres & 1 \\
\hline 5 & $P Q$ & Pronator quadratus & 1 \\
\hline 6 & ECR_LO & Extensor carpi radialis longus & 12 \\
\hline 7 & ECR_BR & Extensor carpi radialis brevis & 12 \\
\hline 8 & ECU & Extensor carpi ulnaris & 12 \\
\hline 9 & FCR & Flexor carpi radialis & 12 \\
\hline 10 & $\mathrm{FCU}$ & Flexor carpi ulnaris & 12 \\
\hline 11 & PL & Palmaris longus & 12 \\
\hline 12 & FDS5 & Flexor digitorum superficialis (pinky finger) & 21617 \\
\hline 13 & FDS4 & Flexor digitorum superficialis (ring finger) & 21314 \\
\hline 14 & FDS3 & Flexor digitorum superficialis (middle finger) & 21011 \\
\hline 15 & FDS2 & Flexor digitorum superficialis (index finger) & 278 \\
\hline 16 & FDP5 & Flexor digitorum profundus (pinky finger) & 2161718 \\
\hline 17 & FDP4 & Flexor digitorum profundus (ring finger) & 2131415 \\
\hline 18 & FDP3 & Flexor digitorum profundus (middle finger) & 2101112 \\
\hline 19 & FDP2 & Flexor digitorum profundus (index finger) & 2789 \\
\hline 20 & EDM & Extensor digiti minimi & 2161718 \\
\hline 21 & ED5 & Extensor digitorum (pinky finger) & 2161718 \\
\hline 22 & ED4 & Extensor digitorum (ring finger) & 2131415 \\
\hline
\end{tabular}




\begin{tabular}{l|l|l}
23 & ED3 & Extensor digitorum (middle finger) \\
24 & ED2 & Extensor digitorum (index finger) \\
25 & EIND & Extensor indicis \\
26 & EPL & Extensor pollicis longus \\
27 & EPB & Extensor pollicis brevis \\
28 & FPB & Flexor pollicis brevis \\
29 & FPL & Flexor pollicis longus \\
30 & APL & Abductor pollicis longus \\
31 & OP & Opponens pollicis \\
32 & APB & Abductor pollicis brevis \\
33 & ADPT & Adductor pollicis transversus
\end{tabular}

2101112

2789

2789

124356

2435

435

24356

1243

43

435

435 


\title{
Chapter 3 Muscle excitation profiles in complex musculoskeletal model of human hand with muscle-based active joint stiffness
}

(this chapter is in preparation to be submitted as "Sobinov A, Matthew B, Gritsenko V, Gaunt $R$, et al., Yakovenko S (2019) Muscle excitation profiles in complex musculoskeletal model of human hand with muscle-based active joint stiffness")

\begin{abstract}
Solving the motor control signals for a desired movement is a complex problem that has remained a focus of research interest for more than 50 years. Developing an accurate representation of these underlying command patterns would greatly increase our understanding of motor control principles. From a practical point of view, an understanding of these representations would help us build assistive devices and develop rehabilitation techniques responsive to the desires and specifics of the user. One approach to investigating the principles of motor control is to find an inverse solution to forward models of the musculoskeletal system. By investigating the cost functions and mechanisms that constrain the inverse solution space, we can infer the principles that the central nervous system uses to plan and execute the movement. Here we provide a solution to a detailed model of the human hand with 8 degrees of freedom and 32 musculotendon actuators. We created the forward and inverse models as systems linearly dependent on the input signals focused on the control of 8 degrees of freedom of wrist, thumb and finger joints. The inverse model was used to obtain computed muscle excitations (CMEs) for 17 movements and 390,625 postures, involving control of the wrist, thumb and fingers. To resist perturbations from noise, we introduced desired joint stiffness as a control parameter for the inverse. The mechanism behind the generation of stiffness was mostly explained by agonist-antagonist coactivation during movement (mean $r=0.77$ ) and to a lesser extent during posture maintenance (mean $r=0.6$ ). The forward simulations of CMEs produced the desired movements, but if no stiffness was imposed on the inverse, the joints tasked with maintaining a posture often deviated from their position. These CMEs were used for offline control of a modelled human hand by recorded EMGs from an able-bodied participant. The average mean angular deviation was $10 \%$ of the range of motion for DOFs maintained static throughout the movement and 19\% for dynamic DOFs. To conclude, our approach allowed us to
\end{abstract}


correctly reconstruct postures and torques to control a complex model of a human hand, and it has the potential to be used as part of a myoelectric controller for transradial amputees.

\section{Introduction}

Inversion of biomechanical models of movement to estimate descending motor command has been a focus of research for more than fifty years. Many approaches have been developed to manage the redundancy of the musculoskeletal (MS) system in relation to specific tasks, which was first stated by Bernstein in 1967 (Bernstein, 1967). The same reaching task can be solved by an infinite number of joint configurations, and a joint trajectory can be traced using different sets of motor signals. The space of solutions for a task can be expressed as an uncontrolled manifold (Scholz and Schöner, 1999). Many principles have been found to guide the selection of controlled DOFs, focusing on constraining the manifold with additional cost functions, like metabolic expenditure (Donelan et al., 2001; Todorov and Jordan, 2002; ValeroCuevas et al., 2009), jerk minimization (Flash and Hogan, 1985), neural activity levels (Sussillo et al., 2015), muscle synergies (d'Avella et al., 2006), and active joint impedance (Stroeve, 1999). Other solutions have relied on the observed output to infer the cost functions for movement optimization by sampling a range of them to find the best-fitting (Terekhov et al., 2010; Terekhov and Zatsiorsky, 2011). While very promising, this method has been only applied to simulated, simplified systems. A MS-based approach has been previously used to decode muscle activity during walking with the OpenSim CMC tool (Thelen and Anderson, 2006) using an iterative optimization algorithm. This approach achieved reconstruction of activity profiles similar to the EMG recordings in an offline setting but was unstable for longer simulation times. A reliable method for calculation of muscle activity that is similar to real EMG signals for complex biomechanical models is yet to be developed.

A practical application of the inverse solutions to the field of myoelectric prosthetic control would help to solve major problems with decoding EMG signals. Myoelectric prosthetics began as simple switches, that allowed changing the state of the prosthetic (for example, grip) when electrical activity of a muscle or a group of muscle crossed a predefined activity threshold (Scott and Parker, 1988). An evolution of that control scheme into continuous space yielded the direct control approach, which supplies the level of activity of a single muscle to a single motor in a prosthesis (Ison and Artemiadis, 2014). Its performance was hindered by the difficulty of independent activation of several muscles, and heavy cross-talk between the recorded signals. To negate these problems, machine learning algorithms were used to relate EMG activity 
patterns to the desired control signal, like speed or position of a joint, or assume an array of static postures (Graupe et al., 1977; Englehart and Hudgins, 2003; Ciancio et al., 2016; Nieveen et al., 2017; Resnik et al., 2018). An alternative approach for myoelectric control relies on the biomimetic simulation of an intact MS structure to translate recorded muscle activity into commands for the prosthesis (Abboudi et al., 1999; Eilenberg et al., 2010; Crouch and Huang, 2016). The benefit of such models is the transparency of the control scheme, generalizability to each specific patient by constraints on the MS simulation, and minimal training required. The complexity of the approach comes from several sources. First, there is a need to correctly identify which muscles are the sources of the recorded EMG signals, which can be especially challenging in an amputee. Second, muscles that are not being recorded need to have signals provided. Third, the low-amplitude posture-stabilizing signals can be potentially discarded during EMG processing. Estimation muscle excitation profiles from the desired movement has the potential to negate these problems by bridging the gap between the modelled hand and the subject's control signals.

In this study, we describe a novel method for obtaining the computed muscle excitations (CMEs) from kinematics using an anatomically accurate MS model of a generic human hand (Sobinov et al., 2019; Boots et al, 2019). In addition to a commonly used metabolic cost function, we specify desired joint stiffness and formulate the problem in a computationallyefficient linear fashion. We evaluate the effect of the desired joint stiffness on the obtained profiles of CMEs and investigate the mechanisms that induce stability within the model. Using forward simulations of the obtained CMEs, we investigate the benefit of induced stiffness in the model. Then we used surface EMGs from a human subject to reconstruct the movements offline through a transformation between CMEs and the recorded signals.

\section{Methods}

The methods describe the dataset that was used for the study, the forward control model of a complex musculoskeletal system, and inverse solution to it. The model consisted of a sequence of transforming the spatiotemporal pattern of muscle activity into the muscle forces and joint torques that were then passed through a physics engine to calculate movement kinematics (Figure 3-1, top). Within this forward transformation, we simulated calcium dynamics with a standard excitation-contraction coupling algorithm (Winters, 1995). The muscle force was calculated using a Hill-type muscle model (Zajac, 1989). This step required accurate reconstruction of the posture-dependent muscle length and velocity, which was achieved with 
polynomial approximations (Sobinov et al., 2019). Together, this sequence of forward calculations could generate movement from muscle activity within 2 ms which allowed real-time performance. The inverse solution to the forward model calculated the computed muscle excitations (CMEs) from the kinematics (Figure 3-1, bottom). Redundancy of the inverse model solution space was addressed with two additive cost functions that represented metabolic expenditure and joint stiffness. Finally, the methods are provided to use the CMEs in forward control of a hand prosthesis.

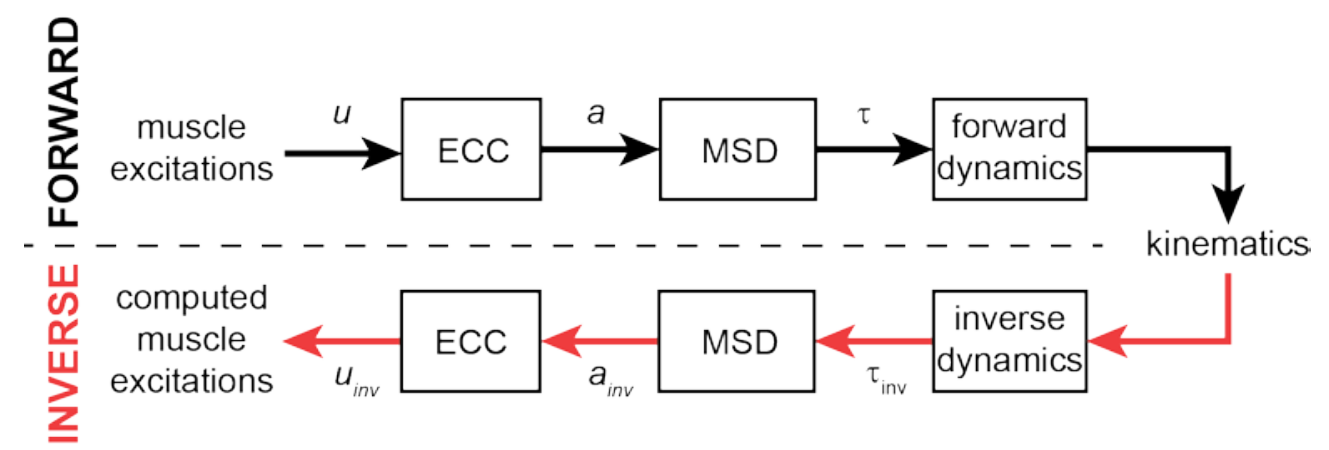

Figure 3-1. Schematic of the steps in forward (black) and inverse (red) control of the musculoskeletal model.

$u$ is a vector of musculotendon unit excitation levels (Equation 3-1); a - musculotendon unit contraction levels (Equation 3-2); $\tau$ - torques at all DOFs applied to a physics model; $\tau_{\text {inv }}-$ inverse torque around 8 DOFs; $a_{i n v}$ - inversely-obtained contraction levels for 32 muscles; $u_{i n v}$ - computed muscle excitations; ECC: excitation-contraction coupling; MSD: musculoskeletal dynamics.

\section{Model}

We simulated control of a human hand containing 17 segments: forearm, palm, 12 phalanxes and three thumb segments, that were connected with 16 joints and 18 degrees of freedom (DOFs, Table 3-2). We simulated 32 musculotendon actuators each spanning up to 6 DOFs. Relationship between MS kinematic variables (moment arms and musculotendon lengths) and the hand posture was obtained from a previously published model (Sobinov et al., 2019; Boots 
et al, 2019). Values for the muscle force properties were obtained from (Boots et al., 2019). Table 3-3 provides a description of all muscles with their parameters.

Although in the forward model we simulated 18 degrees of freedom (DOFs), we focused on the performance of 8 DOFs: wrist flexion-extension and pronation-supination, thumb carpometacarpal flexion-extension and abduction-adduction, and fingers 2 through 5 metacarpophalangeal flexion-extension. In the rest of the chapter we will refer to them as controlled DOFs (cDOFs). We chose these DOFs because they represented differential control of wrist and each individual digit enough to produce recognizable movements of the hand. Proximal interphalangeal (PIP) joints were omitted, because internal hand muscles that are important for their control (Li et al., 2000) were not modelled in this study.

\section{Static Kinematic Dataset}

We intended to explore the whole range of motion of the model with the static dataset and common hand movements with dynamic dataset. The static dataset consisted of all possible combinations of 5 postures of each of 8 cDOFs investigated in the analysis, totaling $5^{8}=390,625$ postures.

\section{Dynamic Kinematic Dataset}

Dynamic kinematic dataset contained 7 types of common hand movements, including grasping, flexion-extension and thumb control. Each movement type could start in several wrist rotation positions (total 17 movements) as described in Table 3-1. For example, pronated grasp movement started with the hand in a neutral pronated posture and a $250 \mathrm{~ms}$ pause, which was followed by a $500 \mathrm{~ms}$ movement, and another $250 \mathrm{~ms}$ pause. The times were selected to represent the movement time of a modern prosthetic hand through a range of motion (Belter and Dollar, 2011), and allow periods of posture maintenance to test stability. The kinematics for the movements were simulated to have smooth bell-shaped velocity profiles, cover $90 \%$ of range of motion of each DOF and sampled at $100 \mathrm{~Hz}$ rate (Beggs and Howarth, 1972; Georgopoulos et al., 1981).

\section{Table 3-1. Dynamic movements.}

This table lists all simulated movements with a description of corresponding artificial kinematics. Wrist pronation-supination position column describes wrist position during the movement whenever that DOF is constant or starting position whenever the movement requires 
change in pronation-supination angle. Time column describes total time given for the movement. Each movement was prefaced and ended with a $250 \mathrm{~ms}$ pause included in the value of the Time column. The wrist f-e, wrist p-s and fist f-e movements (marked *) included a 500 ms pause in the neutral position in the middle of the movement.

\begin{tabular}{|c|c|c|c|}
\hline Type of movement & $\begin{array}{l}\text { Wrist pronation- } \\
\text { supination position }\end{array}$ & Time, s & Description \\
\hline Grasp & $\begin{array}{l}\text { Neutral } \\
\text { Pronated } \\
\text { Supinated }\end{array}$ & 1 & $\begin{array}{l}\text { All fingers move from fully extended } \\
\text { state to fully flexed and back. } \\
\text { Simultaneously, the thumb moves } \\
\text { from extended adducted position to } \\
\text { flexed abducted and back. }\end{array}$ \\
\hline Grasp with wrist & Neutral & 1 & $\begin{array}{l}\text { Same as grasp, but the wrist flexion- } \\
\text { extension DOF moves from neutral } \\
\text { to fully flexed and back. }\end{array}$ \\
\hline Thumb opposition & $\begin{array}{l}\text { Neutral } \\
\text { Pronated } \\
\text { Supinated }\end{array}$ & 1 & $\begin{array}{l}\text { Thumb moves from flexed-adducted } \\
\text { position to flexed-abducted and } \\
\text { back. }\end{array}$ \\
\hline Thumb pinch & $\begin{array}{l}\text { Neutral } \\
\text { Pronated } \\
\text { Supinated }\end{array}$ & 1 & $\begin{array}{l}\text { Thumb moves from extended } \\
\text { adducted position to flexed } \\
\text { abducted and back. }\end{array}$ \\
\hline Wrist f-e* & $\begin{array}{l}\text { Neutral } \\
\text { Pronated } \\
\text { Supinated }\end{array}$ & 2 & $\begin{array}{l}\text { Wrist moves from neutral to flexed, } \\
\text { then back to neutral, maintains the } \\
\text { posture for } 500 \mathrm{~ms} \text {, then to } \\
\text { extended, then back to neutral. }\end{array}$ \\
\hline Wrist $p-s^{*}$ & Neutral & 2 & $\begin{array}{l}\text { Wrist moves to fully pronated, back } \\
\text { to neutral, maintains the posture for }\end{array}$ \\
\hline
\end{tabular}




\begin{tabular}{l|l|l|l}
\hline & & & $\begin{array}{l}500 \mathrm{~ms}, \text { then to fully supinated, } \\
\text { back to neutral. }\end{array}$ \\
\hline Fist f-e* & Neutral & 2 & $\begin{array}{l}\text { Same as wrist f-e but holding all } \\
\text { fingers and thumb in a fist instead of } \\
\text { neutral. }\end{array}$ \\
& \begin{tabular}{l} 
Supinated \\
\hline
\end{tabular} & & \\
\hline
\end{tabular}

The kinematics of both datasets were inverted to obtain the CMEs. To explain the inverse model, we will first review the model of forward control of MS system.

\section{Dynamic Control of Musculoskeletal Model}

We used standard excitation-contraction coupling (ECC) transformation to capture the dynamics of calcium-mediated contraction $(a)$ in response to neuromuscular excitation $(u)$. We modelled ECC as a first-order differential equation (Winters, 1995; Thelen, 2003):

\section{Equation 3-1}

$$
\dot{a}=\left\{\begin{array}{c}
\frac{u-a}{t_{d}}(0.5+1.5 a), u \leq a \\
\frac{u-a}{t_{a}} \cdot \frac{1}{0.5+1.5 a}, u>a
\end{array},\right.
$$

where $u$ is the excitation level of a muscle (input), $a$ is the contraction level of the muscle (output), activation and deactivation time constants $t_{a}$ and $t_{d}$ were $10 \mathrm{~ms}$ and $20 \mathrm{~ms}$, respectively, similar to constants for fast muscles in (Winters, 1995). Both excitation and contraction are normalized variables bounded between 0 and 1. Activation time constant describes how fast the contraction reaches the level of excitation, when excitation (control signal) is higher than contraction. Similarly, deactivation time constant describes how fast the contraction level goes down to the excitation level, if excitation is lower than contraction.

The level of muscle activation and its force were related through a Hill-type muscle model (Zajac, 1989). The model describes how the maximum force that a muscle can produce depends on its length and speed (Zajac, 1989; Gillard et al., 2000; Yakovenko et al., 2004). Equation 3-2 describes these relationships in a vector form for $\mathrm{U}=32$ muscles.

\section{Equation 3-2}

$$
F_{\mathrm{m}}(L, \dot{L}, t)=F_{\text {max }} \cdot F_{L}(L) \cdot F_{V}(\dot{L}) \cdot a(t)+F_{\text {pass }} \cdot F_{P}(L)
$$


where $t$ is time; $L=\left(L_{1}(\Theta), \ldots, L_{\mathrm{U}}(\Theta)\right)^{T}$ are the musculotendon lengths of $\mathrm{U}$ muscles; $\dot{L}=$ $\left(\dot{L}_{1}(\Theta, \dot{\Theta}), \ldots, \dot{L}_{\mathrm{U}}(\Theta, \dot{\Theta})\right)^{T}$ is the change of musculotendon length as a function of time; $F_{\max }=$ $\left(F_{\max , 1}, \ldots, F_{\text {max }, \mathrm{U}}\right)^{T}$ and $F_{\text {pass }}=\left(F_{\text {pass }, 1}, \ldots, F_{\text {pas }, \mathrm{U}}\right)^{T}$ are maximum active and passive forces of the muscles, respectively; $F_{L}$ is the relationship between musculotendon length and its maximum force produced (Equation 3-3); $F_{V}$ is the relationship between musculotendon force and the speed of change of its length (Equation 3-4); $F_{P}$ is the passive muscle force (Equation 3-5); $a=\left(a_{1}(t), \ldots, a_{\mathrm{U}}(t)\right)^{T}-$ contraction level of the muscle. Here and later $A \times B$ is a cross product, $A \cdot B$ is elementwise multiplication.

\section{Equation 3-3}

$$
F_{L}(L)=2.5 \cdot L_{\text {norm }}(L)-1.25 \cdot L_{\text {norm }}^{2}(L)
$$

where $F_{L}$ is the relationship between musculotendon length and its maximum force produced; $L_{\text {max }}=\max _{\Theta} L(\Theta)$ and $L_{\text {min }}=\min _{\Theta} L(\Theta)$ are the maximum and minimum lengths of musculotendon units; $L_{\text {norm }}(L)=\frac{L-L_{\min }}{L_{\max }-L_{\min }} \in[0,1]$ is the musculotendon length of the muscles normalized to the range of each muscle.

\section{Equation 3-4}

$$
F_{V}(\dot{L})=\left\{\begin{array}{c}
1+\left(\frac{1-\exp \left(-0.425 \cdot \dot{L} / L_{R}\right)}{1+\exp \left(-0.425 \cdot \dot{L} / L_{R}\right)}\right), \dot{L} \leq 0 \\
1+0.8\left(\frac{1-\exp \left(-0.425 \cdot \dot{L} / L_{R}\right)}{1+\exp \left(-0.425 \cdot \dot{L} / L_{R}\right)}\right), \dot{L}>0
\end{array},\right.
$$

where $F_{V}$ is the relationship between musculotendon force and the speed of change of its length; $\dot{L}=\frac{d L}{d t}=\left(\dot{L}_{1}(\Theta, \dot{\Theta}), \ldots, \dot{L}_{\mathrm{U}}(\Theta, \dot{\Theta})\right)^{T}$ is the change of musculotendon length as a function of time; $L_{R}=\left(L_{\max }+L_{\min }\right) / 2-$ mean length of the musculotendon units.

\section{Equation 3-5}

$$
F_{P}(L)=\left\{\begin{array}{c}
\frac{\exp \left(2 \cdot \frac{L-L_{\text {pass }}}{L_{\max }-L_{\text {min }}}\right)-1}{\exp (1)-1}, L>L_{\text {pass }}, \\
0, L \leq L_{\text {pass }}
\end{array}\right.
$$

where $F_{P}$ is the passive muscle force; at muscle length $L_{\text {pass }}=\left(L_{\text {pass }, 1}, \ldots, L_{\text {pass }, \mathrm{U}}\right)^{T}$ and longer, the passive muscle force contributes to the force production of the musculotendon unit. 
Muscle contraction produces a pulling force at the points of attachment of the musculotendon unit to the skeletal system. The force pulling on the bone segments produces rotational force (torque) at each DOF that a muscle crosses. The magnitude of this torque depends on the distance to the DOF's axis of rotation (its moment arm):

\section{Equation 3-6}

$$
T(\Theta, L, \dot{L}, t)=\mu(\Theta) \times F_{\mathrm{m}}(L, \dot{L}, t)
$$

Where $T=\left(\tau_{1}, \ldots, \tau_{\mathrm{M}}\right)^{T}$ is torque produced at $\mathrm{M}=18$ DOFs; $\Theta=\left(\Theta_{1}, \ldots, \Theta_{\mathrm{M}}\right)^{T}$ are generalized local coordinates of the model (angles of all DOFs).

The torques calculated via Equation 3-6 were sent to the MuJoCo HAPTIX physics engine (Kumar and Todorov, 2015) simulating a physiological model of a human hand and forearm in real time to produce kinematics. The simulation software allowed extracting kinematics (joint angles) during simulations, which were then used to assess produced trajectories. The model is available upon request.

\section{Inverse Model}

The inverse model described each step of the forward transformation in reverse (Figure 3-1). In the first step, inverse torques were obtained from kinematics and the physical model of human hand segments using MuJoCo Pro inverse dynamics (Todorov et al., 2012). In the second step, the linear system describing the relationship between the inverse torque and muscle activity (Equation 3-6) was solved. The formula was obtained by substituting Equation 3-2 into Equation 3-6 and moving all terms containing contraction a to the right side, everything else to the left side and leaving only the equations corresponding to $M_{c}=8$ controlled DOFs.

\section{Equation 3-7}

$$
T_{1}=T_{a c t} \times a,
$$

where $T_{1} \triangleq T_{i n v}-T_{\text {pass }}=T_{i n v}-\mu \times\left(F_{\text {pass }} \cdot F_{P}\right)$ is the torque we are trying to produce with muscle contraction levels; $T_{i n v}$ is the inverse torque; $T_{\text {pass }}$ is the passive muscle torque; $T_{a c t} \triangleq$ $\mu^{\prime} \cdot\left(E \times\left(F_{\max } \cdot F_{L} \cdot F_{V}\right)\right)$ is the active muscle torque; $E=(1, \ldots, 1)^{T}$ with length $\mathrm{M}_{\mathrm{c}}$. In a static case, $\dot{L}=0$ and $F_{V}=1$. 
The third step in the inverse model was concerned with ECC. The contractions in non-static cases were transformed into muscle excitations via an analytical inverse of the Equation 3-1 (Equation 3-8). In the static case, inverse to ECC was omitted and excitation levels were equal to contractions.

\section{Equation 3-8}

$$
u=\left\{\begin{array}{c}
\dot{a} \cdot \frac{t_{d}}{0.5+1.5 a}+a, \dot{a} \cdot \frac{t_{d}}{0.5+1.5 a} \leq 0 \\
\dot{a} \cdot t_{a}(0.5+1.5 a)+a, \dot{a} \cdot \frac{t_{d}}{0.5+1.5 a}>0
\end{array},\right.
$$

where $\dot{a}=\frac{d a}{d t}$ - first time derivative of the contraction level, all other symbols as in Equation 3-1. The values of excitations $u$ after Equation 3-8 needed to be constrained between 0 and 1.

The difference in the forward and inverse models lies in the second step, transformation between joint torques and muscle contractions. In the forward model, torques are analytically calculated from muscle contractions, while in the inverse model, muscle contractions are a result of numerical optimization in the form of linear pseudoinverse.

\section{Resolving Redundancy of Inverse Model}

For the model with 8 controlled DOFs and 32 muscles, $M_{c}=8$ and the length of $u$ is $U=32$; therefore, there are more equations than variables in Equation 3-7 and there can be more than one solution of the pseudoinverse. We constrained the solution space by adding cost functions to the system. Equation 3-7 is linear on activation level which provides a benefit of using efficient bounded least-squares algorithms to obtain the solution (Byrd et al., 1999). To maintain this linearity when adding cost function, we decided to have all cost function adhere to the same linear structure: $C_{1}=C_{2} \times a$. In the pseudoinverse algorithm, contraction levels were bounded between 0 and 1. To balance between finding the solution to Equation 3-7 and accommodating the cost functions, additive cost functions coefficients ( $A$ and $B$ ) were normalized together to be between 0 and 1. It was done by estimating the approximate maximum expected value for each equation in the system describing the cost function, and then dividing by that number. We used the following assumptions to precompute these values: $a=1 ; F_{L}=1 ; F_{V}=1 ; F_{P}=1$.

Two cost functions were added to Equation 3-7. The first cost function was always included in the inverse and focused on reducing the metabolic cost of the movement: $0=F_{\max } \times a$. This formulation of the metabolic cost favored using muscles with lower maximum forces, assuming 
other parameters (e.g. their moment arms) were the same. A cost function that imposes a desired joint stiffness level was added to ensure stability of movements (see below).

\section{Active Muscle Stiffness}

We added a constraint on the inverse solutions to regulate the resistance to potential perturbations of the controlled system. Static resistance to perturbations is commonly called the stiffness of the system, by analogy with electrical circuits (Hogan, 1984). Assuming the perturbation is an instant displacement from the current trajectory, a stable control signal would produce compensating torques that push the joints back. As an example, consider a joint with one DOF and two antagonistic muscles, one flexor and one extensor (Figure 3-2), maintaining a static posture at joint angle $\theta$. While the posture is being maintained, the net torque at the joint is zero $\left(\tau_{n e t}(\theta)=0\right)$. If the joint is stable, a displacement in the positive direction $(\hat{\theta}=\theta+\delta \theta)$ would lead to negative net torque pushing the joint back $\left(\tau_{\text {net }}(\hat{\theta})<0\right)$. Similarly, the negative displacement $(\check{\theta}=\theta-\delta \theta)$ causes positive compensation $\left(\tau_{n e t}(\check{\theta})>0\right)$. Stated more generally, partial differential of the net torque is negative $\left(\delta \tau_{\text {net }} / \delta \theta<0\right)$ in a stabilizing system. The exact value of the negative partial differential is the definition of stiffness and was used to measure the stiffness of the system:

\section{Equation 3-9}

$$
\mathrm{K}_{\mathrm{i}}=-\frac{\delta T_{i}}{\delta \Theta_{i}}
$$




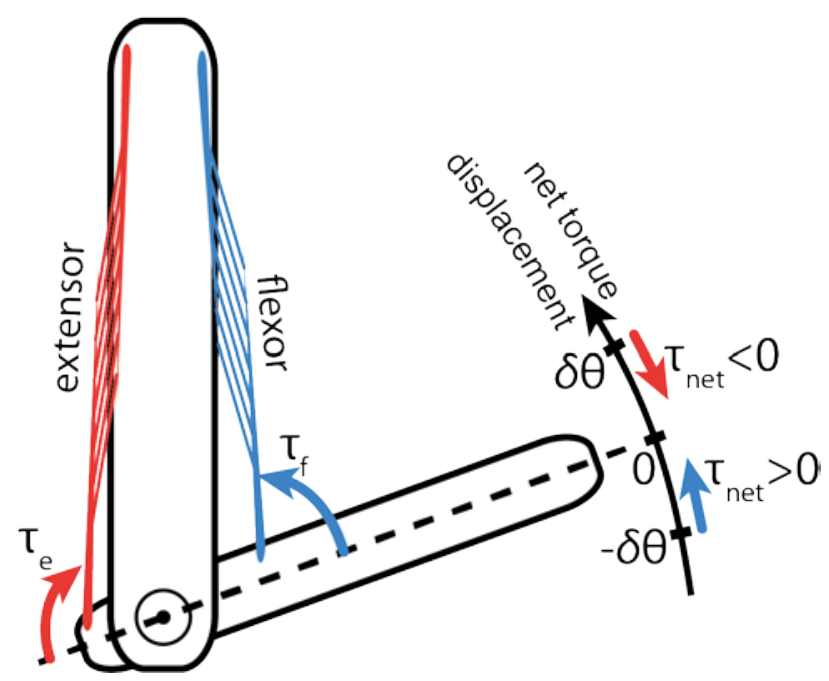

Figure 3-2. Schematic of active muscle stiffness for one DOF and two muscles.

Two antagonistic muscles are pulling on a segment producing flexion and extension torques at the DOF: $\tau_{f}$ and $\tau_{e}$. Together with gravitational torque (not shown) they produce a net torque at the DOF $\tau_{n e t}$. In this task of maintaining a posture $\tau_{f}$ and $\tau_{e}$ are such that $\tau_{n e t}=0$. If the DOF is displaced by $\delta \theta>0$, a stiff configuration of muscle activity would lead to the net torque becoming negative: $\tau_{n e t}(\theta+\delta \theta)<0$, which pushes the DOF back to the original state. Similarly, in case of a negative displacement, $-\delta \theta$, net torque in a stiff system would be positive and push the joint back.

To construct an additive cost function to the inverse model, Equation 3-6 was substituted into Equation 3-9 to produce Equation 3-10, which is linear on muscle contraction a and was appended to Equation 3-7. This cost function was omitted when solving for any desired stiffness (Figure 3-3, Figure 3-4, Figure 3-5, Figure 3-8, and Figure 3-10).

\section{Equation 3-10}

$$
\begin{gathered}
K_{i}^{*} \cdot \Delta \Theta_{i}-D_{i}\left(T_{\text {pass }}\right)-D_{i}\left(T_{\text {inv }}\right)=D_{i}\left(T_{2}\right) \times a, \\
D_{i}(T)=T_{i}\left(\Theta+\frac{\Delta \Theta_{\mathrm{i}}}{2}\right)-T_{i}\left(\Theta-\frac{\Delta \Theta_{\mathrm{i}}}{2}\right)
\end{gathered}
$$

where $\Delta \Theta_{i}=\left(d \Theta_{1}, ., d \Theta_{j}, ., d \Theta_{M}\right)$ is the linear displacement along the DOF $j: d \Theta_{j}=0.01$. Range of Motion of DOF $(j)$ if $j=i$, and $d \Theta_{j}=0$ if $j \neq i ; D_{i}(T)$ is a linear operator that calculates 
a finite difference of torque $T$ across one DOF $i$. Differential of the inverse torque was assumed to be zero: $D_{i}\left(T_{i n v}\right)=0$, for the purposes of decreasing the computational load of obtaining the inverse. This equation was calculated for each degree of freedom $i$ ( $\mathrm{M}_{\mathrm{c}}=8$ equations) and added to Equation 3-2.

There is limited data available on the distribution of active stiffness values of hand joints in healthy individuals. We obtained a vector of normal stiffnesses $\mathcal{K}^{*}$ by setting the value for the wrist flexion-extension DOF to $0.7 \mathrm{Nm} /$ rad based on (Leger and Milner, 2000), and scaled $\mathcal{K}_{i}^{*}$ for other DOFs in proportion to the mass of the segments distal to the DOF (Table 3-2). When the stiffness of the model as a whole was analyzed (Figure 3-3, Figure 3-8, Figure 3-9, Figure 3-10), the desired stiffness is reported in units of $\mathcal{K}^{*}$. To test the responsiveness of the system to the imposed stiffness, in Figure 3-4, Figure 3-5, Figure 3-6 and Figure 3-7 the range of desired stiffness for all DOFs was set to be from 0 to $2 \mathrm{Nm} / \mathrm{rad}$ with $0.5 \mathrm{Nm} / \mathrm{rad}$ increments.

\section{Measuring Agonist-Antagonist Coactivation}

Two muscles are antagonistic if they have an opposing action on a DOF, as shown, for example, on Figure 3-2 with a flexor and extensor pulling in opposite directions. In a modelled system antagonists are described by opposite signs of their moment arms around a joint. Because of the multidimensional and realistic anatomy captured in this model, we have multiple agonists and multiple antagonists for each DOF. There are many possible ways to define coactivation between muscles. We decided to interpret the minimum of the drive supplied to agonists and antagonists as a common descending control signal. Mathematically, the agonistantagonist coactivation at a time point was defined as a minimum of the average excitation of agonists and average excitation of antagonists (Equation 3-7). When agonist-antagonist coactivation level is zero, there is no active muscle force pulling in either direction. When it reaches 100\%, all agonists and antagonists are fully active, and their neural inputs are cancelling out.

\section{Equation 3-11}

$$
\Omega_{i}=\min \left(\operatorname{mean}\left(u_{j}, \forall j: \mu_{i j}<0\right),\left(\operatorname{mean}\left(u_{j}, \forall j: \mu_{i j}>0\right)\right)\right)
$$

where $\Omega_{i}$ is the level of coactivation of agonists and antagonists around DOF $i$. 


\section{Hardware Setup}

The described forward and inverse models were implemented in MATLAB 2018b (MathWorks, Inc.) and ran on Intel Core i5 $2.3 \mathrm{GHz}$ processor with 16 GB DDR4 RAM. The code for simulations is available from authors upon request.

\section{Experimental Setup and EMG Processing}

To test whether the CMEs can be used for prosthetic control, we performed a human experiment. The able-bodied subject was equipped with 16 surface Delsys Trigno EMG targeting 14 muscles and recording with $2000 \mathrm{~Hz}$ sampling frequency. The following muscles

were targeted: biceps brachii (wrist supinator), flexor carpi ulnaris and flexor carpi radialis (wrist flexors), three heads of flexor digitorum superficialis (finger flexor), pronator teres (wrist pronator), extensor carpi radialis and extensor carpi ulnaris (wrist extensors), two heads of extensor digitorum (finger extensor), abductor pollicis longus (thumb abduction and extension), abductor pollicis brevis (thumb abduction and flexion), flexor pollicis longus (thumb abduction and flexion), extensor pollicis brevis (thumb adduction and extension), opponens pollicis (thumb abduction and flexion). The subject was asked to perform 10 repetitions of each movement from Table 3-1 that represent common hand usage and include dynamic and static control of wrist, fingers and thumb. The repetitions were performed in quick succession with sound identifying the start of every movement and a video instruction playing each repetition. The subject gave informed consent, and the protocol was approved by the local ethics committee (IRB Protocol \#1311129283). The recorded EMG signals were processed offline as follows: demeaned, rectified, thresholded and low-pass filtered at cut-off frequency $10 \mathrm{~Hz}$ (2 ${ }^{\text {nd }}$ order Butterworth). Threshold for EMG activity was identified visually from the distribution of raw signal, by detecting a separation between noise and bursting EMG. EMG was resampled at $100 \mathrm{~Hz}$ to match the frequency of kinematic dataset. Principle components (PCs) describing 99\% of variance were extracted from the processed EMGs, yielding $\mathrm{N}_{\mathrm{EMG}}=12 \mathrm{PCs}$. Their scores described presence of each PC at each time point.

The EMG was then used to control the modelled hand in an offline setting through the CMEs obtained from inverse model. Similarly to EMGs, PCs describing 99\% of variance were extracted from CMEs, yielding $\mathrm{N}_{\mathrm{CME}}=22 \mathrm{PCs}$. The scores of CMEs and EMGs were then used to obtain $\mathrm{N}_{\mathrm{CME}}$ by $\mathrm{N}_{\mathrm{EMG}}$ matrix $\mathrm{R}$ relating each CME principle component to each EMG principle component with multiple linear regression. 


\section{Equation 3-12}

$$
P C A_{\text {score }}(C M E)=\mathrm{R} \times P C A_{\text {score }}(E M G)
$$

where $R$ is $N_{C M E}$ by $N_{E M G}$ matrix relating principle components of CMEs and EMGs, the $P C A_{\text {score }}(C M E)$ is $\mathrm{N}_{\mathrm{CME}}$ by $\mathrm{N}_{\mathrm{T}}$ matrix with CME principle component scores for each time point, and $P C A_{\text {score }}(E M G)$ is $\mathrm{N}_{\mathrm{EMG}}$ by $\mathrm{N}_{\mathrm{T}}$ matrix with EMG principle component scores of for each time point. Same as with previous linear pseudoinverse, interior-point algorithm was used to calculate each row of R(Byrd et al., 1999)(Byrd et al., 1999). After that, we simulated an EMGcontrolled hand by transforming EMG signals into CMEs with R. The quality of control was measured by estimating deviation from the desired trajectory for each DOF and normalizing to that DOF's range of motion.

Out of 17 movements in the dynamic dataset we removed 5 with substantially worse average performance: thumb pinch in pronated and supinate starting postures, and wrist movements with closed fist. For the remaining 12 movements, 2/10 repetitions with the worst average performance were omitted to account for the expected subject's error in following the rhythm of movements. The principle component analysis and multiple regression were applied to that subset of data and used for Figure 3-9.

\section{Statistics and Comparisons}

For all regression analyses done in this chapter we used conventional method of fitting a linear model to the data and obtaining an F-statistic for significance. We used a very conservative threshold deeming $p$-value $<0.001$ as being significant.

The deviation of the simulated hand from the desired trajectory was measured as an average angular deviation at each DOF. To compare all DOFs together, we normalized the angular deviation to the range of that DOF.

\section{Results}

We obtained CMEs with variable joint stiffness that maintain static postures and produce dynamic movements. CMEs reproduced the desired torque profiles at 8 degrees of freedom with less than $0.15 \%$ error (Figure 3-3). An increase in desired stiffness led to an increase in muscle activity (Figure 3-4, Figure 3-5) and measured stiffness (Figure 3-6). Furthermore, the measured stiffness correlated with the agonist-antagonist coactivation (Figure 3-7). Forward 
dynamic simulations of movements controlled by CMEs yielded $<5 \%$ average angular deviation from the desired trajectory (Figure 3-9). Experiment with controlling the simulated hand by surface EMGs showed that inverse model of musculoskeletal transformation can be used as a step in a hand myoelectric prosthetic control (Figure 3-10).
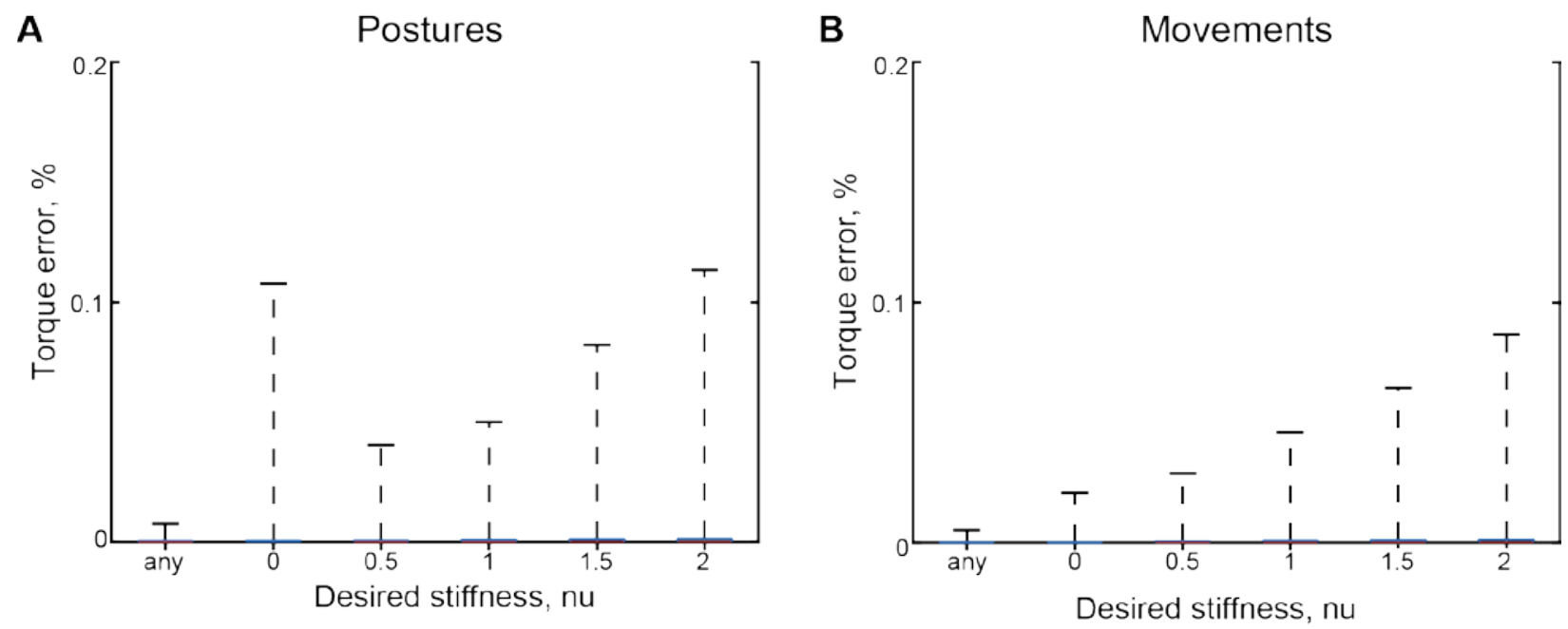

Figure 3-3. Error in torque reconstruction by CMEs.

A. Torque error for posture dataset. B. Torque error for movement dataset. A datum for box plots is a difference between the desired torques $\left(T_{i n v}\right)$ and torques produced by the CMEs calculated through the inverse model from these torques. Torque error was normalized to the maximum static torque at the respective DOF. Boxes indicate quartiles with median (red), whiskers indicate the range of data. Very low errors led to medians being very close to zero and boxes being barely visible.

We obtained CMEs producing 17 movements and 390,625 postures of the hand that involved individual finger, thumb and wrist control (See Dataset in Methods). We focused on the control of 8 DOFs (cDOFs): wrist flexion-extension and pronation-supination, thumb proximal flexion-extension and abduction-adduction, fingers 2 through 5 metacarpophalangeal flexion-extension. These cDOFs were chosen because they allow individual digit control and produce recognizable hand movements. CMEs were obtained for 6 levels of desired stiffness: any (no $\mathrm{K}^{*}$ specified in the inverse) and $K^{*} \in\left(0,0.5 \mathcal{K}^{*}, \mathcal{K}^{*}, 1.5 \mathcal{K}^{*}, 2 \mathcal{K}^{*}\right)$ from both posture and 
movement datasets, where $\mathcal{K}^{*}$ is a set of normally observed joint stiffnesses (see Methods). The CMEs were of expected levels ( $<50 \%$ of maximum) for unloaded hand movements and posture maintenance tasks (Figure 3-4). The increase in desired stiffness $\mathrm{K}^{*}$ lead to rise of CME levels from being mostly zero at $\mathrm{K}^{*}=0$ to median excitation level near $20 \%$ at $\mathrm{K}^{*}=2 \mathcal{K}^{*}$ in both datasets $(p<0.001)$. Although most excitations stayed below $50 \%$, we have observed the full range of activity from 0 to $100 \%$.
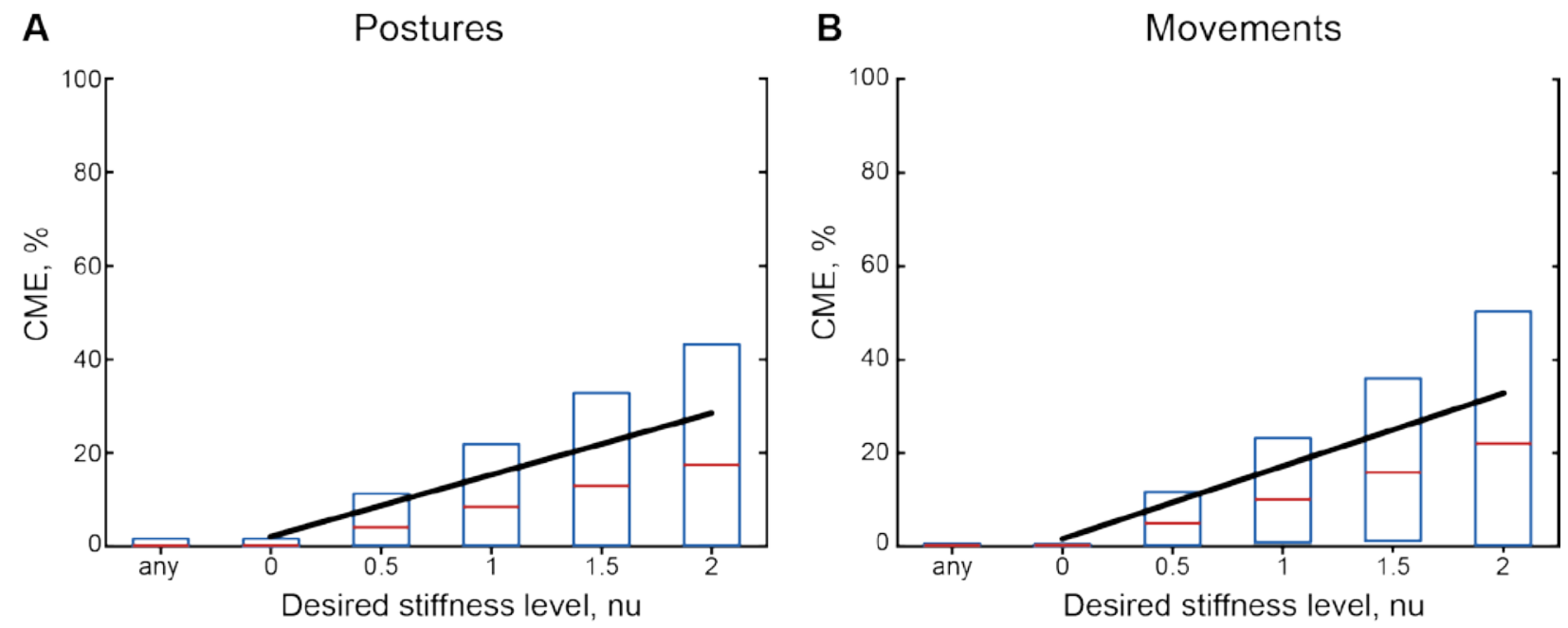

Figure 3-4. Change in CMEs levels with change in desired stiffness.

A. CMEs maintaining static postures, regression line $y=13.20 x+1.96, r=0.40, p<0.001$. B. CMEs producing dynamic movements, regression line $y=15.61 x+1.43, r=0.43, p<$ 0.001. Each box describes quartiles and median (red) of CME levels a with varied stiffness. Boxes marked $\{0,0.5,1,1.5,2\}$ mark CMEs calculated with a stiffness cost function with desired stiffness $\mathrm{K}^{\star}$ equal to the number of normal stiffness levels $\mathcal{K}^{*}$. CMEs for boxes marked 'any' were calculated without a stiffness cost function.

CMEs showed rising levels of agonist-antagonist coactivation with the rise of the desired stiffness levels. As an example, consider excitation of major wrist pronators (pronator teres and pronator quadratis) and supinators (supinator, biceps brachii) at the different postures of the wrist (Figure $3-5)$. With desired stiffness at zero $\left(K^{*}=0\right)$, there was barely any coactivation present: blue and red lines do not have the region where they are both non-zero (Figure 3-5A). 
As the desired stiffness rose, a region of coactivation of pronators and supinators grew. At high desired stiffness $\left(\mathrm{K}^{*}=2 \mathcal{K}^{*}\right)$ antagonistic muscles produced opposing torques that balanced each other out but had a steep negative slope that lead to perturbation resistance. It is important to note that the level of coactivation depended on the posture: when wrist was supinated (Figure 3-5A left side on all subplots) only pronators were active, with the majority of opposing (pronating) torque for stiffness coming from other sources, e.g. gravitational and passive muscle forces. As a quantitative measurement of agonist-antagonist coactivation, we took the minimum of the average excitation of all agonists and all antagonists. This value can be interpreted as a common descending control signal for the muscles, related to regulation of the stiffness, because it produces muscle torques that cancel each other. Figure 3-5B shows the rise in coactivation with the rise in $\mathrm{K}^{\star}(\mathrm{p}<0.001)$. Although the coactivation in this example rose, the median coactivation stayed at zero. 


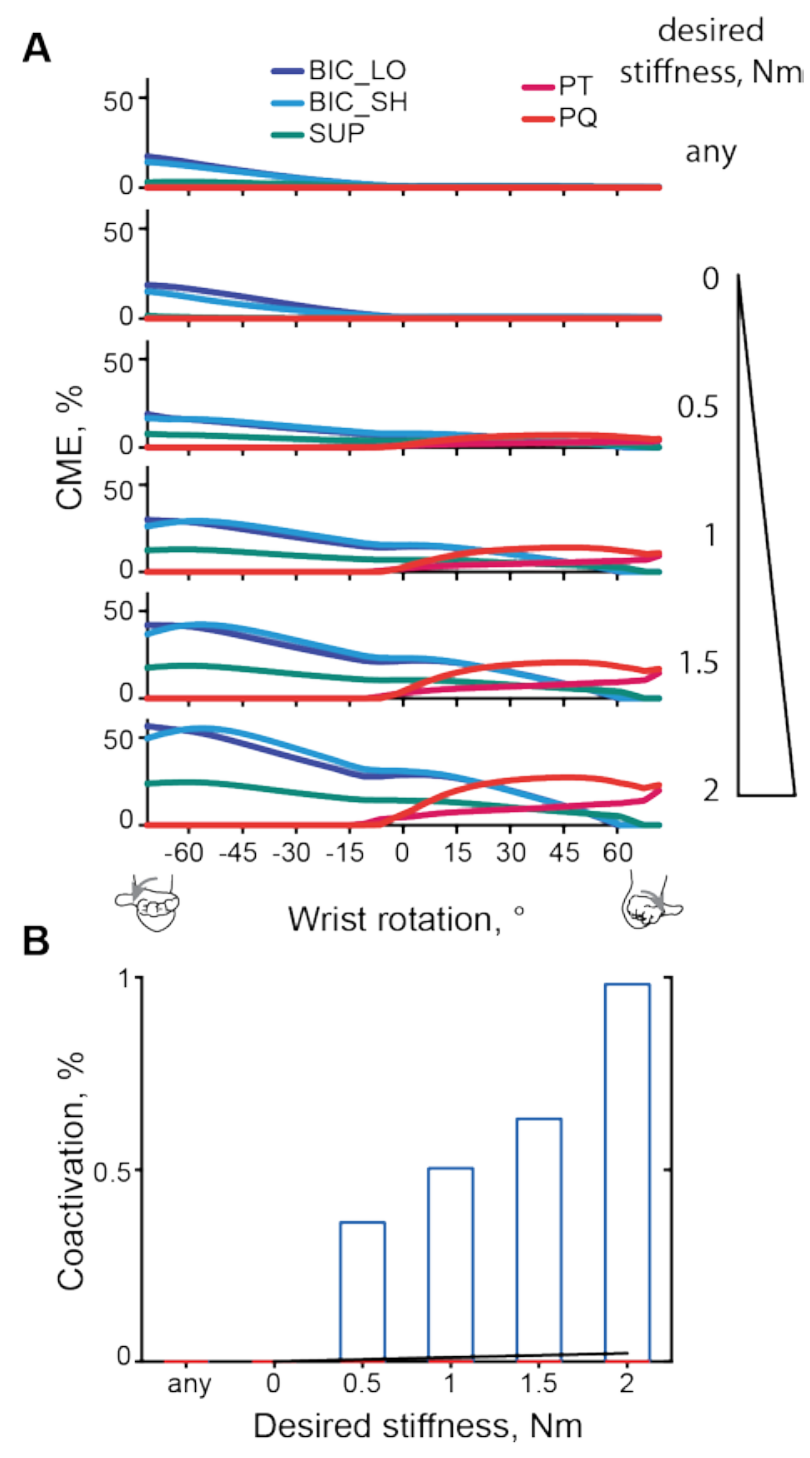

Figure 3-5. Example of CME profiles with different levels of desired stiffness.

A. CMEs of major wrist pronators (red) and supinators (blue) calculated to maintain a range of wrist positions from supinated to pronated. B. Coactivation of the pronators and supinators calculated for each posture using Equation 3-7 for the task shown in subplot A. Each box indicates quartiles and median (red). Regression line $y=0.0106 x+0.0003, r=0.26, p<$ 0.001 .

We found a linear relationship between the desired stiffness $K^{*}$ imposed in the inverse and the measured stiffness $\mathrm{K}$ (Figure 3-6, p<0.001). DOFs split into two visually distinguishable 
groups: (i) wrist and thumb joints; and (ii) finger joints. Wrist and thumb joints had a larger intercept, indicating their intrinsic biomechanical stability, steeper regression slope and higher regression coefficient ( $r$-value). Much shallower regression slope for fingers reflects a weaker relationship between desired and actual stiffness for those joints. Relationship between desired and measured stiffness was stronger in movement dataset (average $r=0.63$ ) than in the static one (average $r=0.35$ ) for fingers.
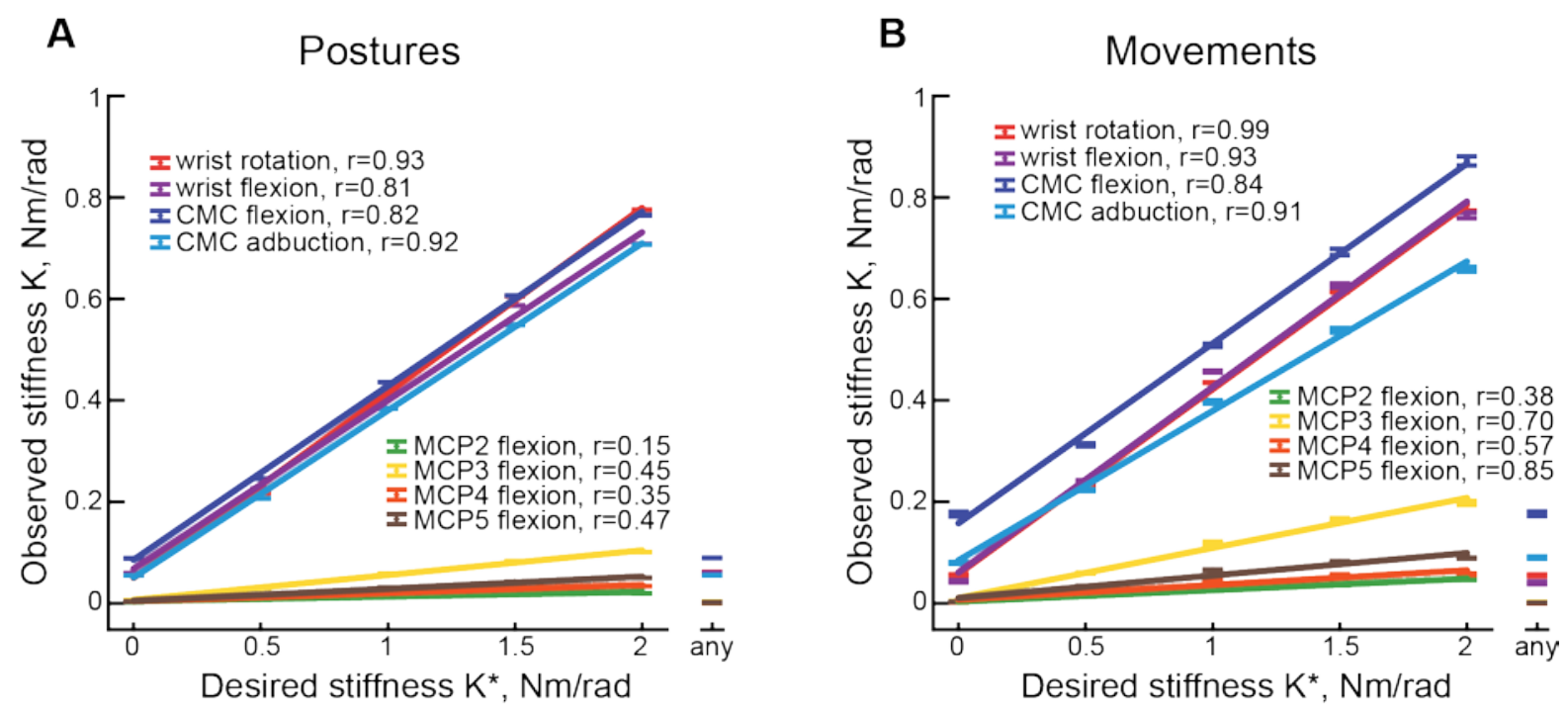

Figure 3-6. Desired $\left(K^{*}\right)$ and observed $(K)$ levels of stiffness in the inverse solutions.

A. Observed stiffness in static postures. B. Observed stiffness in dynamic movements. Observed stiffness $\mathrm{K}$ was measured using Equation 3-5 on the inverse solutions obtained for different levels of desired stiffness $K^{*}$. "Any" marks CMEs computed without stiffness cost function. Error bars show standard error (SE). All linear regressions have $p<0.001$.

We investigated the mechanism behind the generation of stiffness in the model. For each datum of both datasets, we measured the agonist-antagonist coactivation level and stiffness for 8 degrees of freedom. We found a linear relationship between agonist-antagonist coactivation and the measured stiffness in all DOFs (Figure 3-7, p<0.001). The relationship was stronger in the movement dataset, than in the static, as indicated by difference in r-values. On average, $37 \%$ of variance in postures (mean $r=0.60 ; r^{2}=0.37$ ) and $61 \%$ in movements (mean $r=0.77$; 
$\left.r^{2}=61\right)$ was explained by the agonist-antagonist coactivation. The degree to which coactivation contributed to stiffness varied highly between DOFs with the weakest relationship in thumb flexion ( $r=0.39$ and 0.57 in postures and movements, respectively), and strongest in wrist rotation ( 0.75 and 0.99$)$. We observed negative stiffness corresponding to positive torque differential and therefore an unstable subset of positions for several DOFs (Figure 3-7A (a)). A clustering seen in Figure 3-6B (b) as well as other subplots is due to the sampling limitations of the datasets, specifically, 5 levels of desired stiffness. Other sampling limitations that lead to clustering of the data come from the structure of the kinematics: limited number of points sampled for the posture dataset (5 per DOF) and limited number of movements without accounting for their variability between repetitions. Observed behavior suggests that agonistantagonist coactivation plays a crucial role in the generation of joint stiffness. 

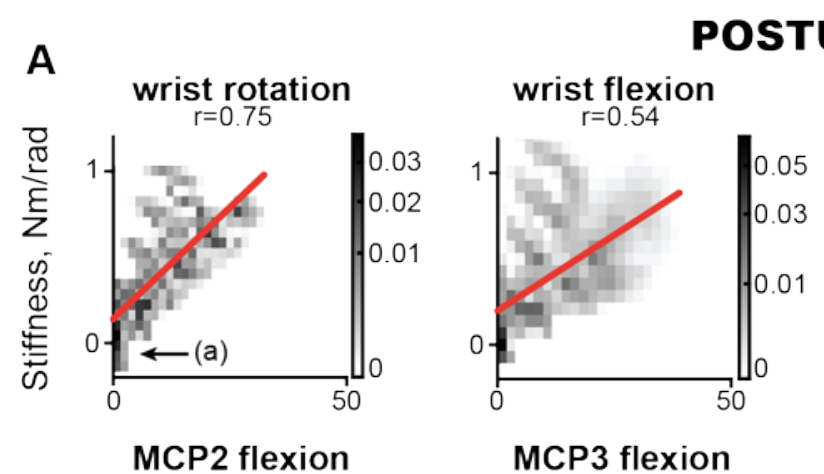

POSTURES
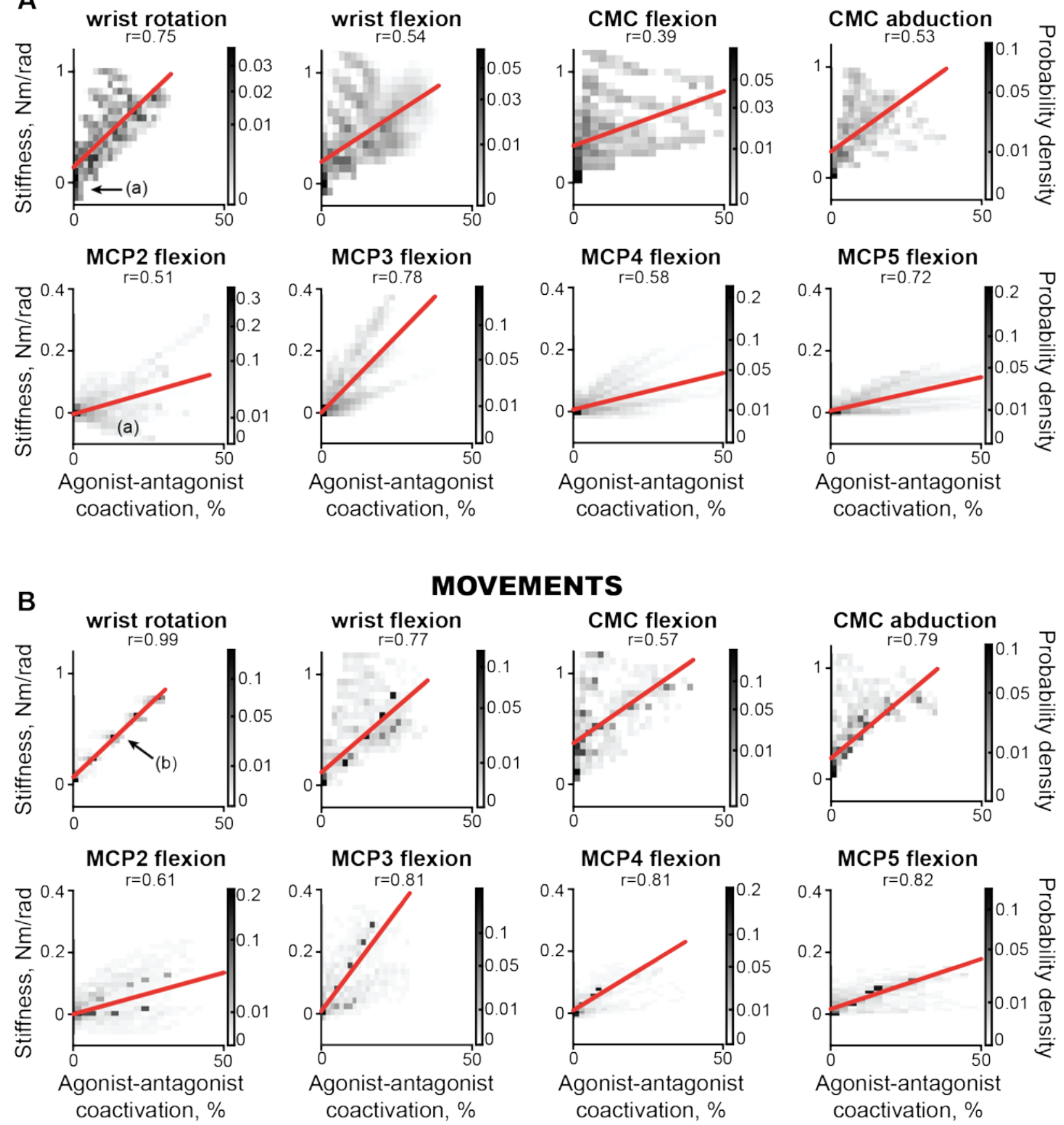

Figure 3-7. Agonist-antagonist coactivation is partially responsible for the joint stiffness.

A. Static posture dataset. B. Dynamic movements dataset. Each subplot contains a heatmap with two-dimensional probability density of coactivation and measured stiffness $\mathrm{K}$ for a specific degree of freedom. The probability density was estimated from a respective (movement or 
posture) dataset and a range of desired stiffnesses: $\left(0 ; 0.5 \mathcal{K}^{*} ; \mathcal{K}^{*} ; 1.5 \mathcal{K}^{*} ; 2 \mathcal{K}^{*}\right)$ (a) marks several cases of negative stiffness, i.e. positive torque differential, which corresponds to unstable movements and postures. (b) marks clustering in probability density heatmaps as a result of limited sampling of levels of stiffness, postures and movements. All regression lines have $p<0.001$.

We performed experiments with CMEs controlling a hand to verify that the CMEs produce the desired movement. The experiments consisted of calculating CMEs from the dataset of movement kinematics, and then using them to simulate the movement (Figure 3-8A). We obtained CMEs for 17 movements with 6 levels of desired stiffness, then simulated the forward dynamics and compared the resulting kinematics to the desired trajectories. Figure 3-8B shows an example of a grasping movement being produced by CMEs with 4 levels of desired stiffness. CMEs computed without a specified stiffness or computed with desirably unstable behavior $\left(K^{\star}=0\right)$ deviated from the desired trajectory at DOFs that were intended to stay static and did not return (marked 'unstable'). The inverse torques at those DOFs were very close to zero, because the neutral posture of the wrist is very close to equilibrium of pronation-supination. Small inverse torques lead to negligible activity of muscles acting on that DOF, and instability in control. When the stiffness was added to the solution, the model maintained the posture (Figure $3-8 \mathrm{~B} \mathrm{~K}^{\star}=\mathcal{K}^{*}$, $\mathrm{K}^{*}=2 \mathcal{K}^{*}$ ). Increased desired stiffness in the inverse lead to rise of the CME levels (Figure 3-8C), as was expected from previous results. Several distinctive behaviors were observed that are characteristic of muscle activity in a movement (Wachholder, 1928; Angel, 1974; Wadman et al., 1979). First, wrist pronators and supinators (Figure 3-8C green shade) displayed constant levels of activity through the movement, maintaining the wrist position. Second, wrist extensors (ECR, ECU), finger flexors (FDS, FDP), and some of the thumb muscles (FPB, FPL, OP, APB) had a single burst of activity during movement (Figure 3-8C pink shade). Third, FCU, finger extensors (EDM, ED, EIND) and the rest of the thumb muscles (EPL, EPB, APL, ADPT) had two bursts during the movement (Figure 3-8C blue shade). CMEs were able to simulate the desired trajectory, increase in desired stiffness ensured stability at static DOFs, and CMEs showed several physiological traits. 
A
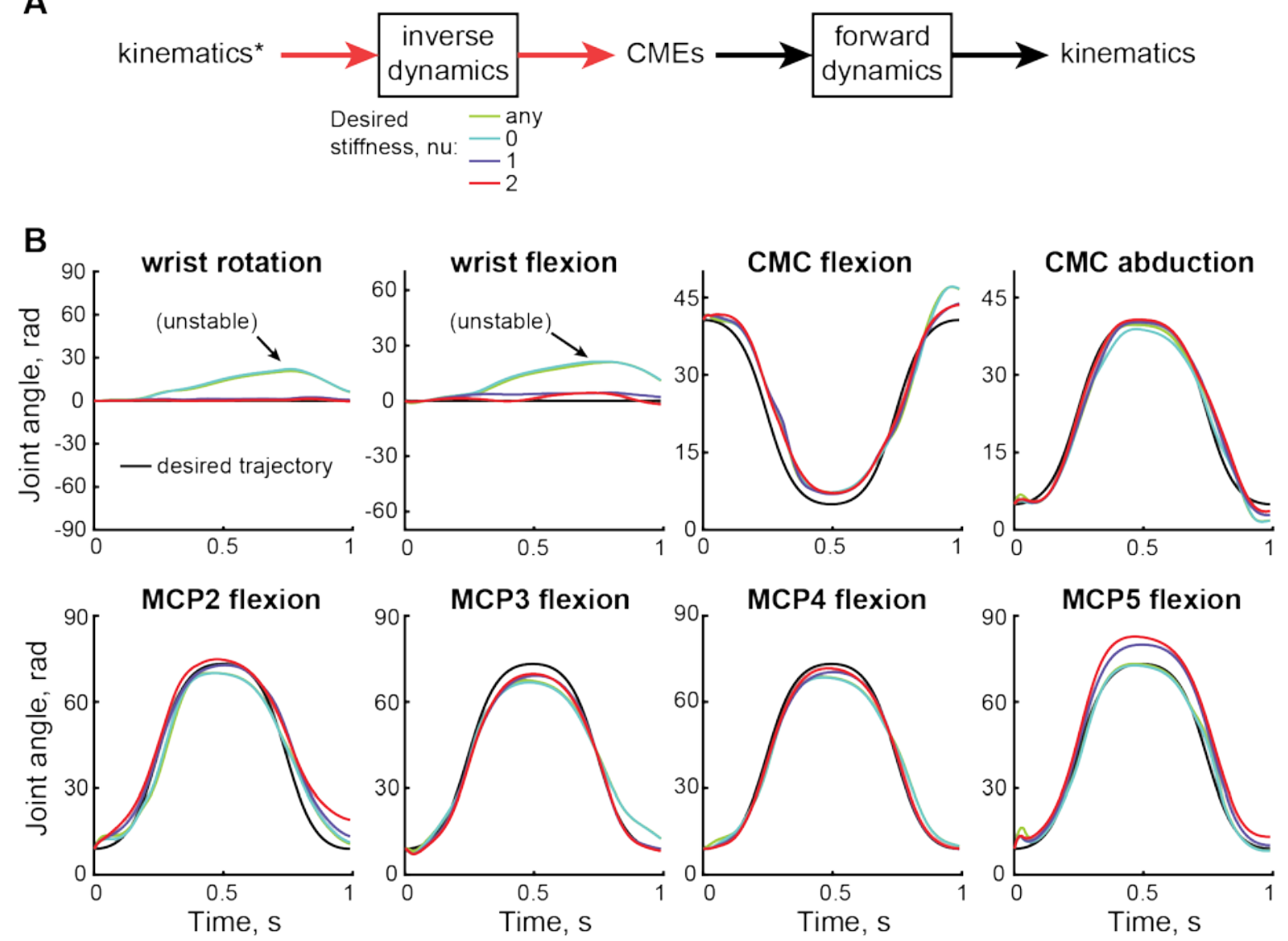

C

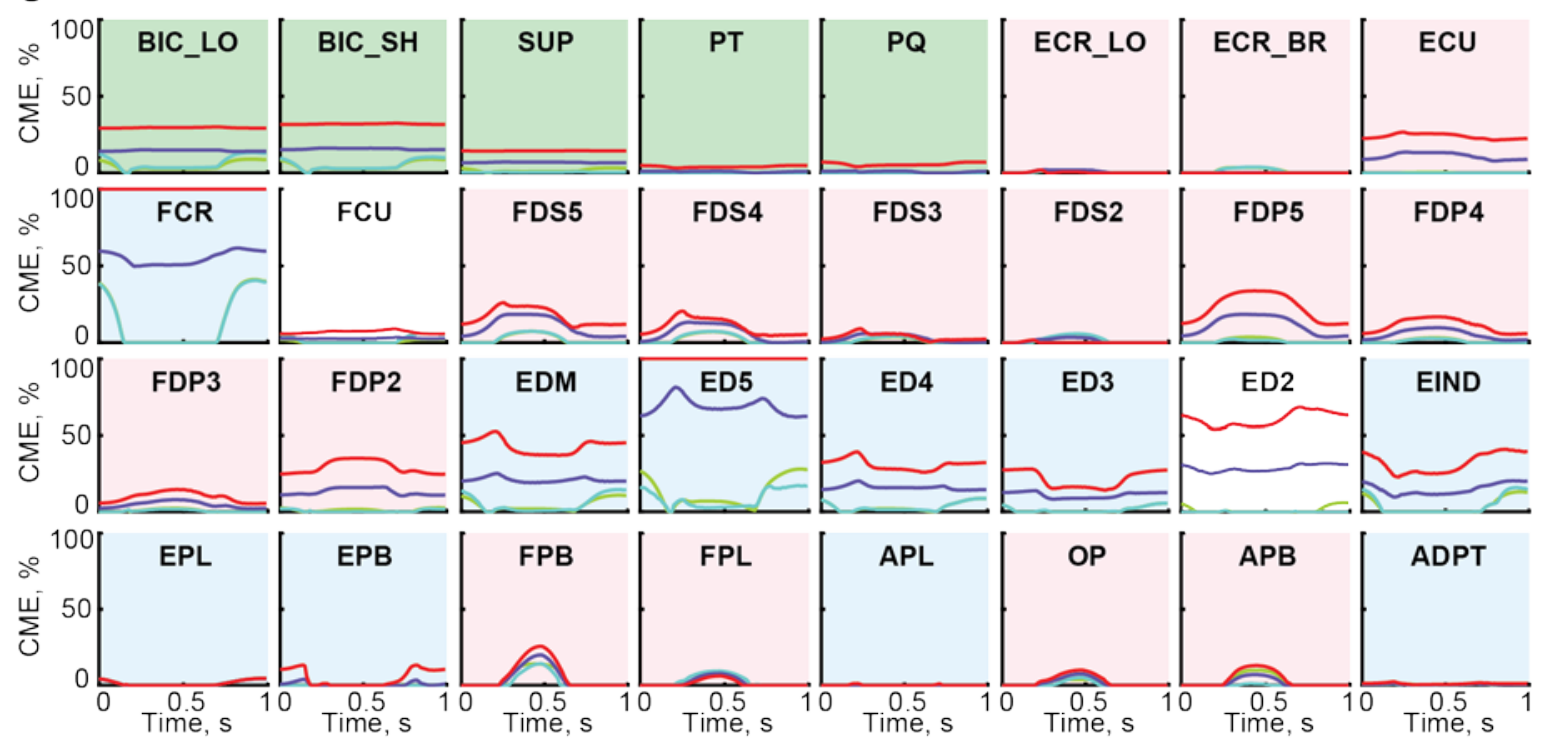

Figure 3-8. Dynamic forward simulation of a grasping movement controlled by CMEs.

A. Schematic of the experiment. Kinematics for a desired movement (kinematics*) were transformed into CMEs through the inverse model with varied level of desired stiffness. The 
CMEs were then used to control the forward model. Color legend for levels of stiffness is the same for all subplots. B. Kinematic traces for a grasping hand movement of 8 DOFs. Wrist is maintained still, all digits fully close and then open. C. CMEs producing the grasping movement. Green shade marks wrist pronators and supinators which maintain a constant level of activity throughout the movement. Red shade marks muscles with a single burst; blue - with two bursts of activity.

We quantified the ability of the CMEs to simulate all the movements from the movement dataset. To estimate the error in a simulation, we measured normalized angular deviation for each DOF during a movement (Figure 3-9). Most DOF trajectories in most movements were reconstructed with errors less than 5\% of ROM. Unstable solutions (0 desired stiffness) showed not significantly worse performance than the solution with any stiffness ( $p=0.053$, two-tailed ttest) or with $50 \%(p=0.09)$. We can reliably control hand motions with low deviations from the movement trajectory with varied stiffness.

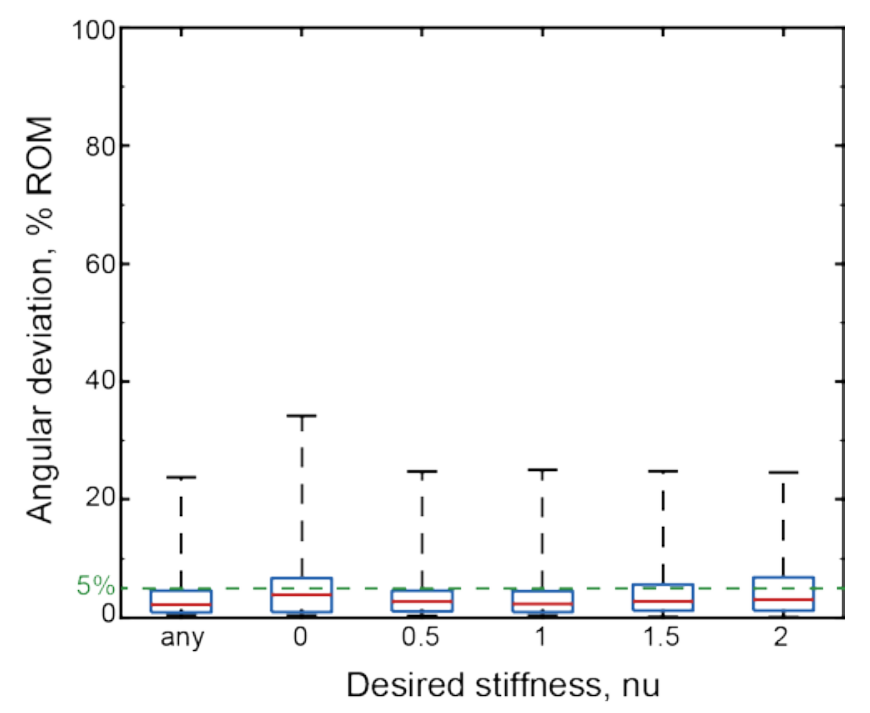

\section{Figure 3-9. Control of movements using CMEs.}

Normalized angular deviation from the desired trajectory during a movement. Angular deviation was normalized to the range of motion at the respective DOF. Box indicates quartiles and median, whiskers indicate the range of data. A datum for the boxplot corresponds to a timepoint in desired kinematics (see Dataset) and a specific DOF. 
We used transformation from recorded to computed muscle activations to simulated hand movement. A participant performed 10 repetitions of 17 movements from the movement dataset, while surface EMG data was recorded from 12 electrodes. After processing, principle components were extracted from the EMG data and the CMEs for the corresponding movements. We used CMEs generated with desired stiffness $\mathrm{K}^{*}=\mathcal{K}^{*}$ based on the values reported in the literature (see Methods). A multiple linear regression between the PCs of EMGs and CMEs was calculated. Then the processed EMG was used to control the hand offline through the linear transformation into the CMEs. Figure 3-10A shows an example of produced kinematic traces for a grasping movement. The shape of the produced kinematics has several characteristics of the desired trajectories. Static wrist DOFs are maintained near their desired posture, deviating less than a quarter of the ROM. Dynamic thumb DOFs cover most of the ROM with a single burst of movement and return to their starting position. The errors of finger joint excursions were the highest, possibly because the movement spanned the whole ROM. The movement was also generated with two bursts instead of one and a noticeable delay. The types of movements that can be controlled by the model depends on the recorded signals from the subject. To estimate the best performance by the model and identify the movements that can be done in this setup with this subject, we selected 12 movements with smallest average deviation error. The five movements removed were: wrist flexion-extension with a closed fist, and two of the thumb movements in pronated and supinated postures, which had visibly worse average performance. From the remaining 12 movements we removed 2/10 repetitions based on the performance to account for subject not synchronizing with the kinematics. After redoing the PCA and regression on the described subset of data, CME profiles were reconstructed with $13 \% \pm 15 \%$ error. In the forward simulation, static DOFs showed smaller errors than the dynamic ( $p<0.001$, one-tailed t-test) with median deviation of 9.77\% ROM being almost two times less than median deviation of dynamic: $18.99 \%$. For DOFs with smaller ROM, e.g. fingers, it corresponds to $9^{\circ}$ and $18^{\circ}$ resolution for postures and movements, respectively. In DOFs with larger DOFs, e.g. wrist, the values reach $18^{\circ}$ and $36^{\circ}$. The simulations of the EMG control were performed in real time, with a loop time needed to estimate the kinematics from the EMGs being less than $2 \mathrm{~ms}$, which allows a $500 \mathrm{~Hz}$ rate of controller loop. 

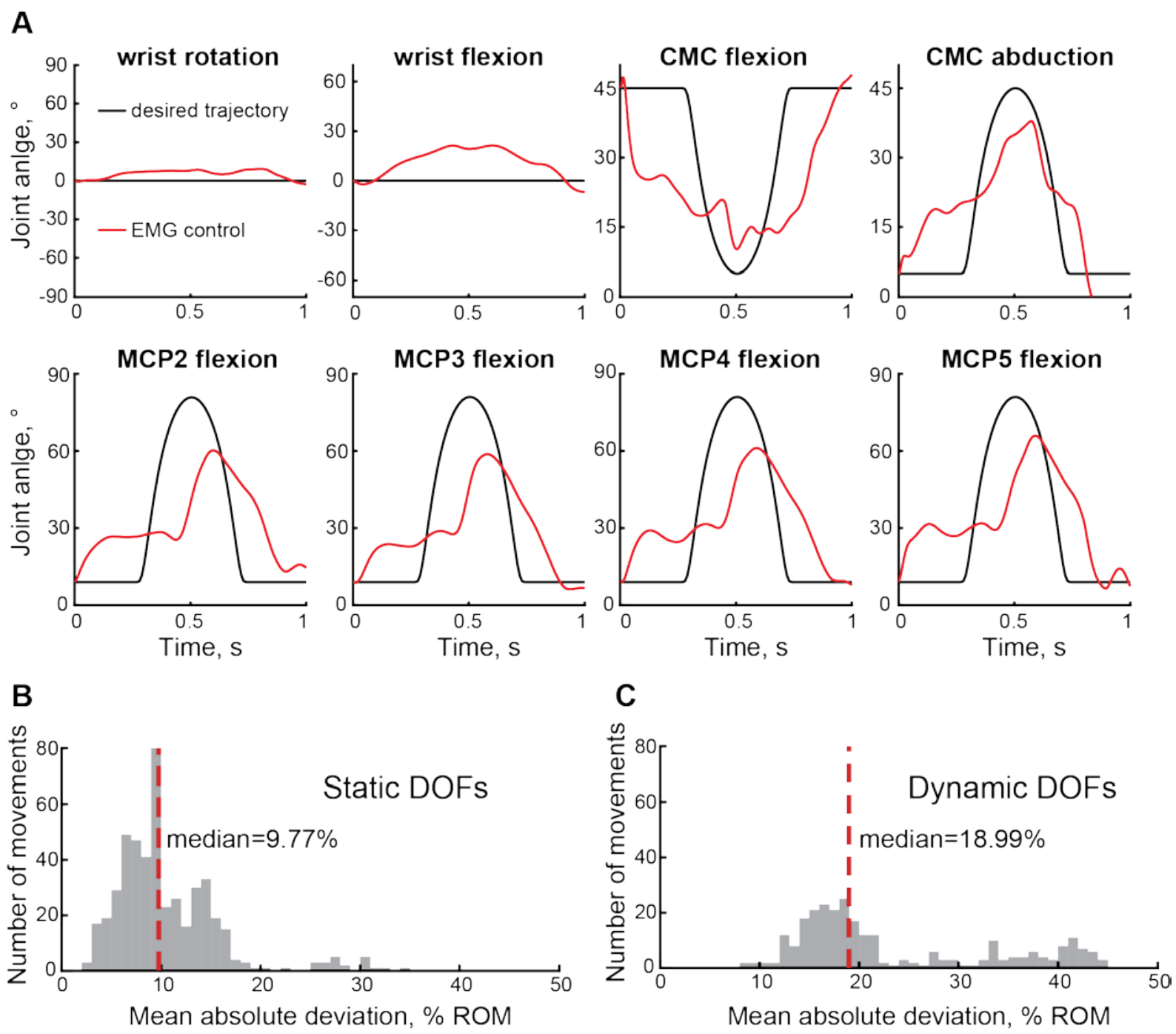

Figure 3-10. Control of dynamic movements using recorded EMG.

A. An example of a grasping movement produced by the MS model. Black - desired kinematic traces, red - controlled by the EMG recorded from a subject following the instructions. B and C: mean absolute deviation of static and dynamic DOFs from the desired postures, respectively, in 12 movements. A datum for the histogram corresponds to an average deviation of a DOF during a movement. The forward simulation model was controlled by EMGs transformed into CMEs via a regression between the principle components of EMGs of a subject performing 8/10 repetitions of 12/17 movements and the principle components of CMEs producing the same movements with constant desired stiffness $\mathrm{K}^{\star}=\mathcal{K}^{*}$. 


\section{Discussion}

We calculated muscle excitation profiles for a complex model of a human hand with varied levels of joint stiffness that maintained postures and produced movements. The stiffness was partially produced by agonist-antagonist coactivation, and partially by the internal biomechanics of joints and passive forces. We showed the potential of using this approach for control of a myoelectric biomimetic prosthesis.

The levels of CMEs increased with the desired stiffness and varied depending on the muscle and the posture. Previous studies have demonstrated an increase in muscle activity with the desire of a subject to resist perturbations (Hogan, 1984; De Serres and Milner, 1991). Variability of muscle activity has also been reported to be between $1 \%$ and $60 \%$ of MVC when maintaining a posture (Antony and Keir, 2010). The observed levels of CMEs are thus physiologically reasonable.

We expected some level of stiffness to be always present at all joints due to the characteristics of the muscle force production. Specifically, when the muscle operates on the 'ascending limb' of the force-length profile the force produced by it increases when it elongates (Zajac, 1989). It has been shown that muscles in humans operate within the ascending limb when the measurements are done in the physiological range of motion (Gillard et al., 2000; Maganaris, 2001), which was reflected in the force-length relationship used in this study. Keeping that in mind, consider a simplistic model system with 1 DOF and two antagonistic muscles (Figure 3-2). Extension of the joint always leads to elongation of the flexor and a corresponding increase in force production. Similarly, the extensor would shorten and produce less force. Together they create a flexion joint torque, which pushes the joint back towards its original position. Similarly, an extension torque would be generated upon flexing the joint. Together these effects result in a negative differential of joint torque, and perturbation-resisting stiffness. When simulating a complex model of the human hand, we observed positions where some of the DOFs could not be stabilized (Figure 3-6). Specifically, when all DOFs were positioned at the extremes of their ROMs, finger joints were often unstable. That could be explained by several key differences between the described 1-DOF model and our model. First, the 1-DOF model ignores changes in the moment arm as a function of joint angle, which might compensate or even invert the changes in muscle length. Second, this simplified model does not include complex dynamics of changes in muscle lengths around a joint containing two DOFs 
(e.g. wrist or thumb in our model) (Sobinov et al., 2019). Both reasons lead to an expectation that there will be postures that could not be stiffened, and that the range of observed stiffnesses would be dictated by MS variables.

In our experiments, we observe the latter as different slopes of regression for wrist, thumb and finger stiffness. The observed and desired stiffness correlated stronger in the movement than in posture dataset for fingers, which suggests that the movements span the stable subset of hand postures. To summarize, we were successfully able to control stiffness of wrist and thumb, and to a lesser extent the fingers with the limits imposed by the MS kinematic variables.

The mechanism behind stiffness production relied on agonist-antagonist coactivation (37\% of variance in postures and 60\% in movements) and on the passive biomechanics of the hand. To explain the mechanism behind a single muscle producing the stiffness at a DOF, let us again consider the 1-DOF system. Even if a flexor or extensor is lacking, the stiffness can be produced by activity of a single muscle, because elongation and shortening of each muscle produces a stabilizing torque. Changing the level of activity of the agonist in this case corresponds to the control of impedance (Hogan, 2002). The resistive antagonistic force to the active muscle can be provided by interaction and gravity torques. Both mechanisms: coactivation and impedance control, are employed by the central nervous system for stabilization of movement.

Currently available biomimetic controllers for prosthetics employ simple 1 to 3-DOF models (Eilenberg et al., 2010; Crouch and Huang, 2016) because the computational load of simulations exceeds the capabilities of the microchips that can be used in a prosthesis. Another common concern is the verification of all MS variables employed in the model (Crouch and Huang, 2016). Due to recent developments in validation techniques (Boots et al., 2019) and the approximation of MS kinematic variables (Sobinov et al., 2019), we were able to simulate 18 DOFs of a human hand with 32 muscles in real time with intended control of 8 of them. Such drastic changes in the available complexity of the model should spark the development of the next generation of biomimetic controllers that do not need to constrain themselves to minimal description.

Evaluation of the CMEs and the inverse model provided an important tool in the analysis and identification of potential problems in the forward biomimetic prosthetic controller. First, it allowed us to establish that the full range of motion is achievable, and showed which parts of 
the MS model prevent a specific posture from being reached. Using such analysis, we identified passive forces that were too strong and were able to adjust them. Second, we found that nonsmooth profiles in the muscle model lead to instability of the model behavior near the transition points. Specifically, using linear piecewise force-length relationship induced oscillations in the forward and the inverse. Third, it allowed us to find DOFs that could not be independently controlled because of the limitations of the model. For example, the MS model in the current form cannot solve for the torques simultaneously at metacarpophalangeal and proximal interphalangeal joints, because they are crossed by the same sets of muscles, but with different moment arms, which creates a linear system without a solution. The separate control of these joints can be achieved when the model includes internal hand muscles that cross only metacarpophalangeal joint. These examples demonstrate the importance of the development of the inverse models to the control schemes for identification of problems.

We were able to relate the CMEs to the recorded surface electrical activity of muscles and produce trajectories closely resembling the desired movements. The resolution of movement reconstruction allowed, on average, to distinguish 10 points of control per DOF for static DOFs and 5 points per dynamic DOFs. An inverse solution that relates the produced CMEs to the recorded EMGs has a capability to account for some of the subject-specific variability in the MS structure. In addition to using the CMEs as a proxy in control of a prosthesis, it can be added to the dynamic control signal to provide the posture-maintaining component that was removed from the EMGs during noise processing. Adding the signal at the control level of the muscle activity is more meaningful than introducing it at the plant level, because it employs stabilization methods innate to biomimetic controllers. Further development is needed to identify a better structure of relation between the CMEs and EMGs.

In the current study we used a vector of desired stiffnesses with values proportional to the inertia of the segment. The choice for the selection was dictated by the limited information available on the stiffness of the hand joints in healthy subjects in posture and movement (Leger and Milner, 2000). The responses of each joint to the imposed stiffness varied, which indicates the need for a better selection of the stiffness values. We plan to address this problem in the future by extracting the stiffness values from the recorded EMG activity by relating it to sets of CMEs with varied levels stiffness. A dedicated set of experiments is needed to achieve that task and evaluate the capability of the inverse solutions to be used in a biomimetic controller. 
To conclude, we used a novel method to compute muscle activity profiles that control a complex 8-DOF and 32-muscle model of a human hand with varied levels of active musclebased joint stiffness. The mechanism behind the generated stiffness was partially explained by agonist-antagonist coactivation, and partially by the interactions of single muscles with the joint biomechanics. The activations were used offline as a part of a biomimetic myoelectric controller which demonstrated their potential to be used for control of a prosthesis for a transradial amputee.

\section{Supplementary information}

\section{Table 3-2. The list of simulated DOFs.}

Name of the DOF is the unique name used in simulations and figures. It has the following structure: $<\mathrm{LIMB}>_{-}<J O I N T>_{-}<M I N>\_<M A X>$. LIMB corresponds to the limb where the joint is located, with 'ra' meaning 'right arm'. JOINT is the joint at which the DOF is located, for example 'wr' for 'wrist'. Digit joints have a number at the end identifying the identifying number of the finger: 1: thumb; 2: index; 3: middle; 4: ring; 5: pinky. The last two suffixes MIN and MAX indicate the anatomical position of the DOF at the minimum and maximum of the range, e.g., "ra_wr_s_p" indicates that the minimum of the wrist pronation-supination DOF at -1.5708 is the supinated posture, and the maximum, 1.5708 - pronated. $\mathrm{K}^{*}$ column lists the desired stiffness used in the inverse cost function. Values for wrist DOFs were taken from (Leger and Milner, 2000). For other DOFs $K^{\star}$ was chosen to be proportional to the mass of the distal segment. For example, for index finger proximal flexion-extension $\mathrm{K}^{*}=<$ mass of proximal, middle and distal phalanx of index finger $>/<$ mass of hand $>* 0.7071$. Masses of each segment are specified in the MuJoCo model.

\begin{tabular}{l|l|l|l|l} 
id & Name & Range, rad & Description & $\mathrm{K}^{*}, \mathrm{Nm} / \mathrm{rad}$ \\
\hline 1 & ra_wr_s_p & $\begin{array}{l}-1.5708 \\
1.5708\end{array}$ & wrist pronation/supination & 0.7071 \\
2 & ra_wr_e_f & $\begin{array}{l}-1.2217 \\
1.2217\end{array}$ & wrist flexion/extension \\
3 & ra_cmc1_f_e & 0.8727 & $\begin{array}{l}\text { thumb proximal } \\
\text { flexion/extension } \\
\text { thumb proximal }\end{array}$ & 0.7071 \\
4 & ra_cmc1_ad_ab & 0.0 .8727 & $\begin{array}{l}\text { abduction/adduction } \\
\text { thumb central flexion/extension }\end{array}$ & 0.0746 \\
5 & ra_mcp1_f_e & -0.78540 & 0.0746
\end{tabular}




\begin{tabular}{l|l|l|l|l}
6 & ra_ip1_f_e & -1.57080 & thumb distal flexion/extension & 0.0309 \\
7 & ra_mcp2_e_f & 01.5708 & index proximal flexion/extension & 0.0880 \\
8 & ra_pip2_e_f & 02.0944 & index central flexion/extension & 0.0569 \\
9 & ra_dip2_e_f & 01.5708 & index distal flexion/extension & 0.0250 \\
10 & ra_mcp3_e_f & 01.5708 & middle proximal & 0.0933 \\
11 & ra_pip3_e_f & 02.0944 & flexion/extension & \\
12 & ra_dip3_e_f & 01.5708 & middle central flexion/extension & 0.0546 \\
13 & ra_mcp4_e_f & 01.5708 & ring proximal flexion/extension & 0.0658 \\
14 & ra_pip4_e_f & 02.0944 & ring central flexion/extension & 0.0385 \\
15 & ra_dip4_e_f & 01.5708 & ring distal flexion/extension & 0.0178 \\
16 & ra_mcp5_e_f & 01.5708 & pinky proximal flexion/extension & 0.0501 \\
17 & ra_pip5_e_f & 02.0944 & pinky central flexion/extension & 0.0269 \\
18 & ra_dip5_e_f & 01.5708 & pinky distal flexion/extension & 0.0129
\end{tabular}

Table 3-3. The list of simulated musculotendon actuators.

Name column describes the short name used in simulations and figures. Full name describes the anatomical name of the muscle and specifies the head of the actuator. DOFs lists all DOFs that each muscle spans by referencing ids from the Table $3-2$. $L_{\min }, L_{\text {pass}}, L_{\max }$ list the minimum length of musculotendon unit, length at which the passive force kicks in, and maximum length (see Equation 3-2). Minimum and maximum length of musculotendon unit were obtained from the raw data and random sampling of the approximating function (Sobinov et al., 2019). Fpass and Fmax list passive and maximum force of the musculotendon actuator (see Equation 3-2 and Boots et al., 2019).

\begin{tabular}{l|l|l|l|l|l} 
id & Name & Full name & DOFs & $L_{\min } L_{\text {pass }} L_{\max }, \mathrm{m}$ & $F_{\text {pass }} F_{\max }, \mathrm{N}$ \\
\hline 1 & BIC_LO & $\begin{array}{l}\text { Biceps brachii long } \\
\text { head }\end{array}$ & 1 & 0.3280 .4330 .445 & 10.92154 .3 \\
3 & BIC_SH & $\begin{array}{l}\text { Biceps brachii short } \\
\text { head }\end{array}$ & 1 & 0.2480 .3540 .366 & 10.92154 .3 \\
4 & SUP & $\begin{array}{l}\text { Supinator } \\
\text { Pronator teres }\end{array}$ & 1 & 0.0680 .0860 .088 & 25.55361 .0 \\
PT & 1 & 0.2170 .2350 .237 & 23.1317 .2
\end{tabular}




\begin{tabular}{|c|c|c|c|c|c|}
\hline 5 & $P Q$ & Pronator quadratus & 1 & 0.0220 .0330 .041 & 12.25168 .2 \\
\hline 6 & ECR_LO & $\begin{array}{l}\text { Extensor carpi } \\
\text { radialis longus }\end{array}$ & 12 & 0.3160 .3460 .349 & 14136.4 \\
\hline 7 & ECR_BR & $\begin{array}{l}\text { Extensor carpi } \\
\text { radialis brevis }\end{array}$ & 12 & 0.3150 .3480 .352 & 17.15167 .1 \\
\hline 8 & ECU & $\begin{array}{l}\text { Extensor carpi } \\
\text { ulnaris }\end{array}$ & 12 & 0.3180 .3340 .336 & 12.25119 .3 \\
\hline 9 & FCR & Flexor carpi radialis & 12 & 0.2870 .3320 .337 & 18.254 .2 \\
\hline 10 & FCU & Flexor carpi ulnaris & 12 & 0.2940 .3270 .331 & 35104.2 \\
\hline 11 & FDS5 & $\begin{array}{l}\text { Flexor digitorum } \\
\text { superficialis (pinky } \\
\text { finger) }\end{array}$ & 21617 & 0.3120 .3650 .371 & 7.3558 .4 \\
\hline 12 & FDS4 & $\begin{array}{l}\text { Flexor digitorum } \\
\text { superficialis (ring } \\
\text { finger) }\end{array}$ & 21314 & 0.3260 .3810 .387 & 8.457 .7 \\
\hline 13 & FDS3 & $\begin{array}{l}\text { Flexor digitorum } \\
\text { superficialis (middle } \\
\text { finger) }\end{array}$ & 21011 & 0.3350 .3930 .399 & 14.7109 .1 \\
\hline 14 & FDS2 & $\begin{array}{l}\text { Flexor digitorum } \\
\text { superficialis (index } \\
\text { finger) }\end{array}$ & 278 & 0.3340 .3860 .391 & 12.6117 .8 \\
\hline 15 & FDP5 & $\begin{array}{l}\text { Flexor digitorum } \\
\text { profundus (pinky } \\
\text { finger) }\end{array}$ & $\begin{array}{l}21617 \\
18\end{array}$ & 0.3290 .3860 .392 & 8.7569 .6 \\
\hline 16 & FDP4 & $\begin{array}{l}\text { Flexor digitorum } \\
\text { profundus (ring } \\
\text { finger) }\end{array}$ & $\begin{array}{l}21314 \\
15\end{array}$ & 0.3440 .4020 .409 & 12.9589 .1 \\
\hline 17 & FDP3 & $\begin{array}{l}\text { Flexor digitorum } \\
\text { profundus (middle } \\
\text { finger) }\end{array}$ & $\begin{array}{l}21011 \\
12\end{array}$ & 0.3560 .4160 .422 & 14.35106 .5 \\
\hline 18 & FDP2 & $\begin{array}{l}\text { Flexor digitorum } \\
\text { profundus (index } \\
\text { finger) }\end{array}$ & 2789 & 0.3470 .4090 .415 & 14.35134 .1 \\
\hline 19 & EDM & $\begin{array}{l}\text { Extensor digiti } \\
\text { minimi }\end{array}$ & $\begin{array}{l}21617 \\
18\end{array}$ & 0.3730 .3950 .423 & 5.2578 .7 \\
\hline 20 & ED5 & $\begin{array}{l}\text { Extensor digitorum } \\
\text { (pinky finger) }\end{array}$ & $\begin{array}{l}21617 \\
18\end{array}$ & 0.3720 .3980 .424 & 1.7526 .2 \\
\hline
\end{tabular}




\begin{tabular}{|c|c|c|c|c|c|}
\hline 21 & ED4 & $\begin{array}{l}\text { Extensor digitorum } \\
\text { (ring finger) }\end{array}$ & $\begin{array}{l}21314 \\
15\end{array}$ & 0.3860 .4140 .441 & 4.2122 .1 \\
\hline 22 & ED3 & $\begin{array}{l}\text { Extensor digitorum } \\
\text { (middle finger) }\end{array}$ & $\begin{array}{l}21011 \\
12\end{array}$ & 0.3960 .4290 .458 & 5.95109 .0 \\
\hline 23 & ED2 & $\begin{array}{l}\text { Extensor digitorum } \\
\text { (index finger) }\end{array}$ & 2789 & 0.3970 .4310 .458 & 3.8552 .9 \\
\hline 24 & EIND & Extensor indicis & 2789 & 0.2310 .2610 .292 & 4.5562 .6 \\
\hline 25 & EPL & $\begin{array}{l}\text { Extensor pollicis } \\
\text { longus }\end{array}$ & $\begin{array}{l}12435 \\
6\end{array}$ & 0.2430 .2740 .294 & 6.65402 .7 \\
\hline 26 & EPB & $\begin{array}{l}\text { Extensor pollicis } \\
\text { brevis }\end{array}$ & 2435 & 0.1680 .1790 .199 & 4.5542 .9 \\
\hline 27 & FPB & $\begin{array}{l}\text { Flexor pollicis } \\
\text { brevis }\end{array}$ & 435 & 0.0790 .1010 .104 & 4.5534 .9 \\
\hline 28 & FPL & $\begin{array}{l}\text { Flexor pollicis } \\
\text { longus }\end{array}$ & 24356 & 0.2300 .2640 .289 & 17.85136 .9 \\
\hline 29 & APL & $\begin{array}{l}\text { Abductor pollicis } \\
\text { longus }\end{array}$ & 1243 & 0.1660 .1810 .198 & 13.65128 .9 \\
\hline 30 & OP & Opponens pollicis & 43 & 0.0580 .0640 .064 & 10.1577 .8 \\
\hline 31 & APB & $\begin{array}{l}\text { Abductor pollicis } \\
\text { brevis }\end{array}$ & 435 & 0.0590 .0740 .076 & 5.2540 .2 \\
\hline 32 & ADPT & $\begin{array}{l}\text { Adductor pollicis } \\
\text { transversus }\end{array}$ & 435 & 0.0320 .0610 .064 & 3.15141 .4 \\
\hline
\end{tabular}




\title{
Chapter 4 Model of a bilateral Brown-type central pattern generator for symmetric and asymmetric locomotion
}

(this chapter is taken directly from my publication "Sobinov A, Yakovenko S (2017) Model of a bilateral Brown-type central pattern generator for symmetric and asymmetric locomotion. $\mathrm{J}$ Neurophysiol:jn.00443.2017 Available at: http://dx.doi.org/10.1101/146993.")

\begin{abstract}
The coordinated activity of muscles is produced in part by spinal rhythmogenic neural circuits, termed central pattern generators (CPGs). A classical CPG model is a system of coupled oscillators that transform locomotor drive into coordinated and gait-specific patterns of muscle recruitment. The network properties of this conceptual model can be simulated by a system of ordinary differential equations with a physiologically-inspired coupling locus of interactions capturing the timing relationship for bilateral coordination of limbs in locomotion. While most similar models are solved numerically, it is intriguing to have a full analytical description of this plausible CPG architecture to illuminate the functionality within this structure and to expand it to include steering control. Here, we provided a closed-form analytical solution contrasted against the previous numerical method. The evaluation time of the analytical solution was decreased by an order of magnitude when compared to the numerical approach (relative errors, <0.01\%). The analytical solution tested and supported the previous finding that the input to the model can be expressed in units of the desired limb locomotor speed. Furthermore, we performed parametric sensitivity analysis in the context of controlling steering and documented two possible mechanisms associated with either an external drive or intrinsic CPG parameters. The results identify specific propriospinal pathways that may be associated with adaptations within the CPG structure. The model offered several network configurations that may generate the same behavioral outcomes.
\end{abstract}

\section{New \& Noteworthy}

Using a simple process of leaky integration, we developed an analytical solution to a robust model of spinal pattern generation. We analyzed the ability of this neural element to exert locomotor control of the signal associated with limb speeds and tested the ability of this simple structure to embed steering control using the velocity signal in the model's inputs or within the internal connectivity of its elements. 


\section{Introduction}

Specialized neural elements in the spinal cord, known as the central pattern generators (CPGs), contribute to the generation of periodic coordinated patterns of locomotor activity (Grillner and Zangger, 1975). Discovered in deafferented preparations, CPGs do not require sensory signals to produce locomotor behavior; however, their pattern is greatly influenced by sensory and descending inputs (Yakovenko, 2011; Prochazka and Ellaway, 2012). Specifically, the direct electrical stimulation of a brainstem structure called the mesencephalic locomotor region (MLR), even in decerebrated animals, produces oscillations in the CPGs and subsequent locomotor behavior (Grillner and Wallén, 1985). This locomotor behavior is characterized by the complex coordinated actions of multiple muscle groups. It is remarkable that a change in either the magnitude or frequency of MLR stimulation can generate all appropriate modifications of these patterns. This increase in stimulation expresses a full repertoire of gaits with continuous transitions, such as from walking to trotting or galloping in over-the-ground locomotion (Shik et al., 1966), or transitioning from slow walking to swimming in amphibians (Cabelguen et al., 2003), which is faster than the walking mode of locomotion. Thus, increasing stimulation input current corresponds to an increase in locomotor velocity.

Many CPG models were developed over the last century (Verzár, 1923; Taga et al., 1991; Bashor, 1998; Yakovenko et al., 2005; Rybak et al., 2006; Markin et al., 2010; Barnett and Cymbalyuk, 2014). Simulated model structure and its parameters are usually derived from observing the motor output patterns or their changes in response to external inputs or naturally occurring variations. These models give rise to the mechanistic descriptions that capture biological organization and the processes; however, they generally start as phenomenological or statistical representations of observed phase variations or timing in the recorded muscle activity. For example, both the limb-based Brown's CPG (Brown, 1911) and the joint-based Grillner's CPG (Grillner, 1981) are similarly founded on the observations of multiple representative electromyographic (EMG) profiles providing insight into the functional organization of this circuitry.

The idea of a CPG as a distributed mechanism that integrates convergent inputs (Grillner and Wallén, 1985) has been supported by both computational and experimental studies. Using calcium imaging, the spatiotemporal activity of rhythmogenic circuitry was found to be functionally distributed with motoneurons in the rostral lumbar and sacral segments of the spinal 
cord (Bonnot et al., 2002; O'Donovan et al., 2005). The spatiotemporal distribution of neural activity throughout the lumbar enlargement with descending control and sensory inputs intact was visualized by combining the anatomical location of the motoneurons with information about their activity during normal locomotion (Yakovenko et al., 2002). This was also supported by observations of independent and coupled recruitment of flexor and extensor rhythmogenic spinal circuits using selective optogenetic approaches (Hägglund et al., 2013). The rhythmogenesis in only flexors or only extensors observed with optogenetics supports the computational observation of a switch-like transition between flexors and extensors (or more precisely, limb protractors and retractors), which identifies them as distinct network elements (Yakovenko et al., 2002). This bilateral, switch-like activation of the motor pools spanning the full rostocaudal extent of the lumbosacral enlargement is likely associated with distributed rhythm-generating networks responsible for this activity.

The integration of feedforward predictions and sensory feedback about ongoing execution is the optimal solution for generating robust control of complex body morphology (Kuo, 2002). Over the course of evolution, the process of optimization within control pathways has likely been concerned with the optimization of locomotion, as this is a central behavior that is essential for animal survival (Yakovenko, 2011). One engineering solution to the problem of computing predictive commands for complex systems is the use of inverse models (Smith, 1957; Wolpert and Ghahramani, 2000). The complex transformation from muscle excitations into movement kinematics could be internalized for inverse solutions that generate appropriate output for the desired kinematic input. It is then not surprising that dedicated rhythmogenic networks for locomotion may be embedding the dynamics of body-ground interactions to solve the problems of intra- and interlimb coordination (Taga et al., 1991; Full and Koditschek, 1999). The accuracy of these embedded neural calculations of MS transformation may be fine-tuned by experience (Wolpert et al., 1998; Bhushan and Shadmehr, 1999; Kawato, 1999; Ijspeert et al., 2013). It is important to acknowledge that sensory feedback pathways may also shape the final output of motor pathways and compensate for dynamics during locomotion. In addition, there is considerable evidence that CPGs integrate sensory inputs together with supraspinal commands to generate changes in the timing and magnitude of locomotor activity (Ijspeert, 2008; Yakovenko, 2011). The inputs from descending pathways may also be phasically modulated by supraspinal interactions to provide appropriate locomotor synergistic coupling, e.g., by the phasic modulation from cerebellum (Arshavsky and Orlovsky, 2016) receiving sensory feedback about ongoing phasic activity via ascending tracts. The theoretical details of interactions 
between multiple descending pathways on the spinal pattern generating circuitry remains to be unknown.

CPG models offer a unique research opportunity to understand the interplay between these neural directives and biomechanical constraints that govern a complex dynamic task. To this extent, we have previously used inverse solutions of a CPG model to infer the nature of descending inputs (Yakovenko, 2011). The surprising result of these simulations was that the input to the CPG was the speed of each limb. Described mathematically as a system of differential equations (Matsuoka, 1985; Schöner et al., 1990; Wallén et al., 1992; Cymbalyuk et al., 2002; Rybak et al., 2006; Yakovenko, 2011), CPG models are hard, even impossible, to solve analytically in the form of known functions and variables. Still, analytical expressions have several advantages over numerical models. Unlike numerical solutions that often suffer from the accumulating errors and inversely related computational load, the analytical solutions are precise within assumptions taken during their derivation. Even though they are also evaluated, their formulation is more efficient and faster than the approximate numerical solutions.

In this study, we developed a method to obtain an analytical solution to one of the simplest implementations of a locomotor CPG using a rate network with continuous variables, not spikes. We used this analytical expression to further test the ability of this circuitry to embed the regulation of phases appropriate for different speeds and control steering with asymmetric gaits. While the identification of pattern generating elements is a considerable challenge in experimental techniques, the function of distributed elements of a CPG can be probed with computational methods that allow us to monitor and manipulate any part of the circuit. We tested two hypotheses in this study: 1) the exact analytical solution exists for a bilateral CPG model implemented with a leaky integration process; 2) the intrinsic circuit redundancy in a CPG can accommodate the expression of asymmetric gait. The function of embedding the asymmetric representations of gait may be relevant for understanding steering and short- and long-term adaptations within spinal systems.

\section{Methods}

\section{Model description}

While a few CPG models of neural activity consider specific ion dynamics using the HodgkinHuxley formulation (Cymbalyuk et al., 2002; Rybak et al., 2006), our model captures gross CPG 
network dynamics, described by T.G. Brown, in a form of gated leaky integration. We expressed the input-output relationship using coupled leaky integrators formulated as a system of ordinary differential equations (ODEs). The system of ODEs can be expressed in matrix form (Equation 4-1), with ipsilateral antagonism expressed as abrupt, non-overlapping state transitions. The state transitions of coupled reciprocal integrators may require complex handling. An event associated with any given state value $\left(x_{i}\right)$ crossing 1 (detected at machine precision) triggers the resetting of the state to 0 and the start of integration for the ipsilateral antagonist. Small perturbations at the transition points could lead to instability. We have solved this problem by allowing only the switching of reciprocal states initiated by the transition from 1 to 0 . The reversal of integration to 0 or negative values does not initiate the integration in the reciprocal state in this model. In Figure 4-1, for example, if the left flexor $\left(x_{1}\right)$ reaches 1 , it resets to 0 and turns off, while the left extensor $\left(x_{2}\right)$ switches on.

\section{Equation 4-1}

$$
\dot{x}=U_{0}+G_{u} u+G_{l} x+G x
$$

where $x=\left(x_{1}, x_{2}, x_{3}, x_{4}\right)^{T}$ - state vector, $U_{0}$ - constant input from intrinsic connections, $G_{u}$ extrinsic input gains, $u$ - extrinsic inputs, $G_{l}$ - leak gains, $G$ - weights for connections between integrators $\left(r_{f f}, r_{f e}, r_{e f}, r_{e e}\right.$ weights in Figure 4-1).

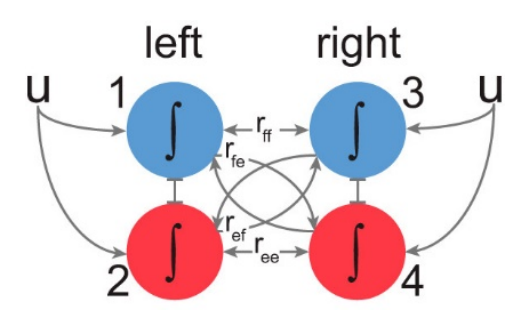

Figure 4-1. Schematic of the bilateral locomotor CPG model.

The oscillatory behavior in each half-center (marked 1-4) was generated through an intrinsic, leaky integrate-to-threshold resetting. This process was also under regulation from intrinsic inputs governed by parameters (rff, rfe, ref, ree). The flexor half-centers (blue) were reciprocally connected to extensor half-centers (red). See Equation 4-1 and Equation 4-2 for details. 
To simplify model parameter space, the parameters were coupled assuming symmetrical organization across the midline, as seen in Equation 4-2. Additionally, the connection between flexors $\left(r_{f f}\right)$ was removed for simulations of walking behavior, where swing phases do not overlap.

\section{Equation 4-2}

$$
U_{0}=\left(\begin{array}{l}
u_{0 f} \\
u_{0 e} \\
u_{0 f} \\
u_{o e}
\end{array}\right), G_{u}=\left(\begin{array}{l}
g_{u f} \\
g_{u e} \\
g_{u f} \\
g_{u e}
\end{array}\right), G_{l}=\left(\begin{array}{l}
g_{l f} \\
g_{l e} \\
g_{l f} \\
g_{l e}
\end{array}\right) \cdot I, G=\left(\begin{array}{cccc}
0 & 0 & 0 & r_{e f} \\
0 & 0 & r_{f e} & r_{e e} \\
0 & r_{e f} & 0 & 0 \\
r_{f e} & r_{e e} & 0 & 0
\end{array}\right)
$$

We used the fixed-step 4th order Runge-Kutta method with $10^{-3} \mathrm{~s}$ precision for forward numerical integration.

The bilateral CPG model produces flexor (swing) and extensor (stance) phases for two limbs in relation to extrinsic input and intrinsic structure. To obtain these phases, Equation 4-1 needs to be integrated in time between the state changes. Numerical integration was previously used (Yakovenko, 2011) to generate swing and stance periods. The same transition points can be calculated analytically by transforming Equation 4-1 into a matrix Cauchy problem and solving a transcendental equation for time of phase change of individual integrators:

\section{Equation 4-3}

$$
z_{1} \cdot \cosh (q \tau)+z_{2} \cdot \frac{\sinh (q \tau)}{q}=z_{3} \cdot e^{-s \tau}
$$

where $z_{1}$ and $z_{2}$ are terms describing interactions between internal structure of the system and input $\left(U_{0}\right.$ and $\left.u\right), z_{3}$ represents the terms with inverse leaks and external inputs, $s$ represents general system leakage, $q$ corresponds to the internal structure of the model (for the detailed description of these terms, see Appendix below).

Using this equation, the periods of activity of flexors and extensors during a step cycle were obtained with an iterative algorithm that was sequentially estimating state changes of each integrator until a full step was completed. Appendix (below) provides the details of derivation for Equation 4-3. 


\section{Cost function}

The CPG model can generate multiple locomotor behaviors as a function of extrinsic inputs and intrinsic interactions (Yakovenko, 2011). Given a desired behavior, e.g. stereotypical symmetrical walking (Halbertsma, 1983), the appropriate CPG parameters were found by optimizing the cost function (Equation 4-4) that expressed the goodness of fit between target (experimental) and simulated patterns. In the symmetrical model, we optimized for 6 different speeds, from 0.1 to $1.5 \mathrm{~m} / \mathrm{s}$ (dashed lines in Figure 4-2), that were generated with 6 values of $u$ (evenly distributed between 0.1 and $1.5 \mathrm{au}$ ). Figure 4-2 shows the quality of simulated solutions for symmetrical walking over a full range of walking speeds.

\section{Equation 4-4}

$$
J_{c}=k_{1} H+k_{2} M+k_{3} O+k_{4} C
$$

where $H$ is the squared difference of simulated and experimental stance and swing periods. The experimental periods were calculated using a best-fit formula obtained empirically with $1 \%$ average error (see Figure 4-6 and Table 2 in Halbertsma, 1983). $M$ is the squared difference of simulated and desired speed ranges that promotes the converging on nontrivial solutions. $O$ is the cost associated with the erroneous coactivation of contralateral flexors. $C$ is the degree of asymmetricity between the simulated speeds of the left and right limbs. All function components were normalized to the domain between 0 and 1 and relative weights $\left(k_{1}, k_{2}, k_{3}, k_{4}\right)=(1,0.7,2$, 0.4). The weights were chosen to represent the relative importance of the captured behavior and be of different magnitudes, which is recommended in optimization. $C$ and $M$ components were removed in simulations intended to produce asymmetrical gait (see Figure 4-6 \& Figure 4-7 in Results). 


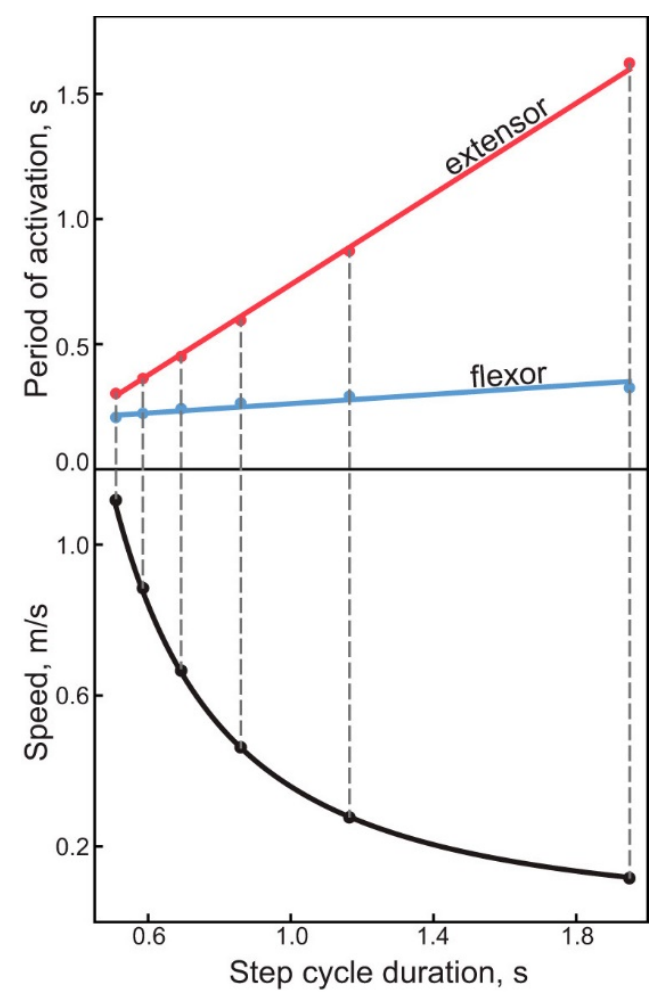

Figure 4-2. Experimental and simulated locomotor phase duration characteristic.

Top: The relationship between the locomotor phase and step cycle duration is shown with points representing the superimposed numerical and analytical solutions for flexor (blue) and extensor (red) phases (Halbertsma, 1983). Bottom: The corresponding simulated speed (black points) is plotted as a function of step duration computed with the exponential best-fit function (black line) (see Fig. 3, in Goslow et al., 1973).

\section{Optimization and parameter perturbation}

Globally optimal sets of parameters were found numerically using a combination of the basinhopping algorithm (Wales and Doye, 1997) in SciPy (Oliphant, 2007) and several constrained local minimizers: the non-linear optimization algorithm COBYLA (Powell, 1964), the truncated Newton algorithm (Nocedal and Wright, 2000), the L-BFGS-B algorithm (Byrd et al., 1995), and Powell's method (Powell, 1964). First, the global optimal parameter set $\left(z^{\star}\right)$ was found. During optimization, the starting value for the basin-hopping algorithm was obtained from a brute force search over the complete parameter space. Other algorithms were then used to optimize parameters sequentially to arrive at the optimal solution $\left(z^{*}=\operatorname{argmin}(J c)\right)$. Second, we created a 
normal multivariate distribution to evaluate the nature of close-to-optimal solutions. For this, the distribution was defined by the mean at $z^{\star}$ and the covariance matrix with the diagonal elements set to $0.01 z^{\star}$ or the equivalent of the standard deviation set at $1 \%$ of the value of the optimal solution. The dataset of $10^{5}$ points was then drawn from this distribution and used in the comparison between the analytical and numerical solutions in Figure 4-3A. Third, the intermediate solutions of the first step corresponding to local minima were selected to determine the full functional range of parameters in the model, excluding sets with large cost values $(J c>10)$. The adjusted for symmetricity range for each parameter is shown as the span of the $y-$ axes in Figure 4-4. Fourth, we used a uniform distribution across the symmetrical full range of parameters to create another dataset of $10^{5}$ values for the analysis of the expanded range comparison shown in Figure 4-3B and C. Fifth, we created the parameter dataset perturbed by $10 \%$ from $z^{\star}$. Similar to step 2 above, we created the normal multivariate distribution with the mean at $z^{*}$ and the covariance diagonal elements set to $0.1 z^{*}$. Sixth, we randomly drew 40 starting seeds and tasked the basin-hopping algorithm (set to 200-iterations for each seed) to repeat the optimization using one of the four local optimization algorithms. This final step in the analysis generated 160 optimal sets for all local algorithms in our analysis. The comparison of parametric distributions is shown for a third of the best solutions in Figure 4-4. The cut of solutions was necessary to reject expected minimization failures with non-converging searches or those terminating with large cost function values.

\section{Phenomenological models of locomotion}

We used several phenomenological models created to describe the relationships between different parameters of stepping during locomotion in our analysis. The relationships between stance and swing phases relative to cycle duration were taken from the study by Halbertsma (Halbertsma, 1983). The relationship between step cycle duration $\left(T_{c}\right)$ and limb speed $(V)$ was taken from the study by Goslow et al. (1973), where $V=\left(1.84 \cdot T_{c}\right)^{-1.68}$ (see Figure 4-2, bottom) (Goslow et al., 1973). Here, we define the limb speed as the scalar rate of progression during one step cycle. The locomotor velocity is then a vector describing the change in body's position as a function of limb speeds. Both studies used best-fit functions to describe data from a small sample of cats; yet, these relationships have been recently confirmed with a large subject pool (Frigon et al., 2015). 
In the analysis of asymmetrical locomotion, we introduced a simple geometrical relationship for walking on a curve. The turn radius $(R)$ of an asymmetric bipedal walk (Equation 4-5) was expressed as a function of hip width $(L)$ and an asymmetry parameter $\alpha=V_{\text {left }} / V_{\text {right }}$ :

\section{Equation 4-5}

$$
R=\frac{L}{|\alpha-1|}
$$

The corresponding heading direction change during a single step can be stated as:

\section{Equation 4-6}

$$
\gamma=\arctan \left(\frac{V_{\text {right }}-V_{\text {left }}}{L} T_{C}\right)
$$

where $y$ denotes the heading direction angle from forward direction; $T_{c}$ - full step cycle period.

\section{Results}

\section{Comparison of analytical and numerical solutions}

In this study, the continuous dynamics between phase transitions was demonstrated with a CPG model expressed as a system of interacting oscillators and solved either numerically or analytically using an iterative algorithm (Equation 4-3). Analytical solutions were validated in simulations producing experimentally observed periods of flexor and extensor activations in overground locomotion (for example, see Figure 4-2). This model was further extended to analyze asymmetric gait and test the ability of this circuit to embed asymmetric gait control.

A high-precision numerical approach carries a processing cost that usually exceeds that of analytical methods. Figure 4-3 shows the comparison of the processing cost between the numerical and analytical solutions for this model (Equation 4-1). The error of evaluating phase transitions with the numerical method (blue line) and the analytical solutions using the rootfinding algorithm (red line) was the same at the precision for numerical integration set to $10^{-3} \mathrm{~S}$ (intersection marked with *, Figure 4-3A and B). The analytical solutions to Equation 4-3, found by expanding the hyperbolic terms, linear to the 9th power, are shown with shades of gray in Figure 4-3. Here, the difference between the analytical and numerical estimations of the time of phase transitions was evaluated with the root mean square metric of simulation quality. Shown 
in Figure 4-3A, the quadratic approximation (gray line marked with a 2) provided similar quality to the analytical solutions (red line), with sets of close-to-optimal parameters (in 1\% vicinity of the optimal set; see step two in section "Optimization and parameter perturbation" in Methods). When the model parameters were chosen randomly from the full range of feasible parameters (steps three and four in Methods), quadratic solutions did not provide desirable precision and performed worse than the numerical method, with other powers only approaching a reasonable threshold of over 10 ms error (Figure 4-3B), which is the order of a motor unit action potential.

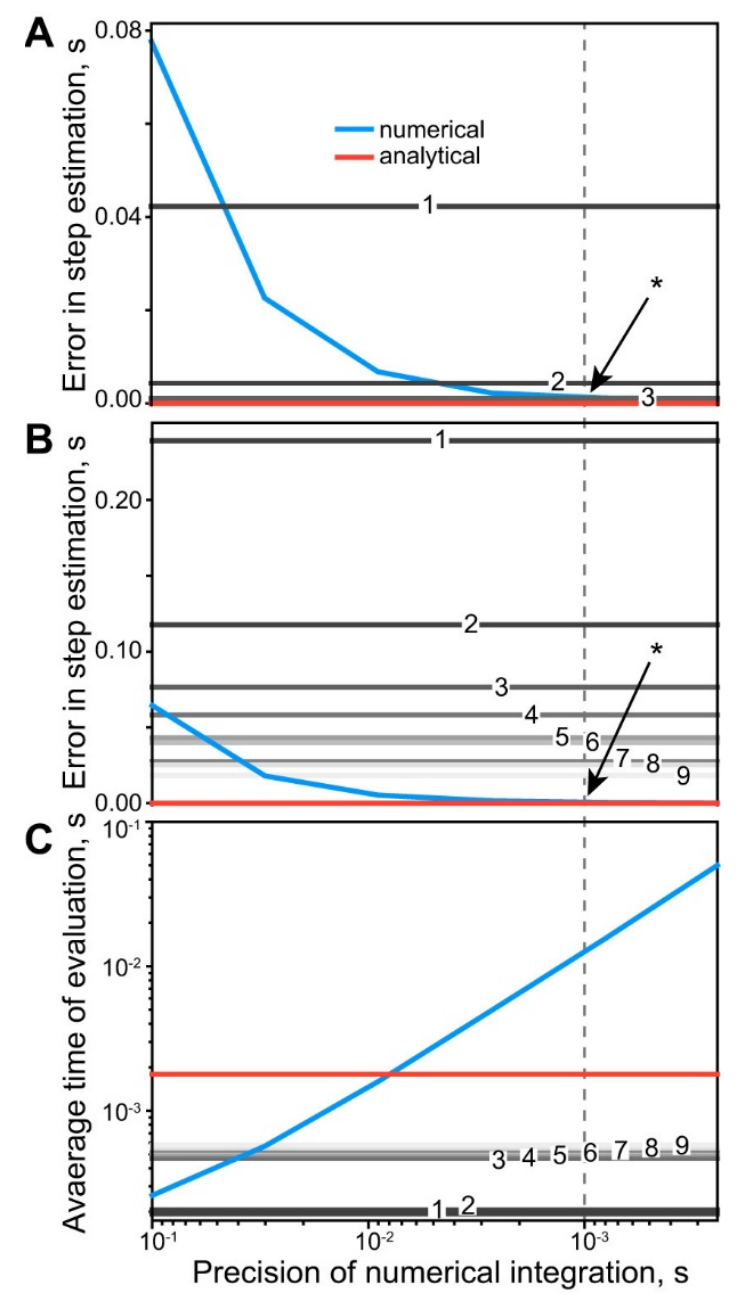

\section{Figure 4-3. The comparison of analytical and numerical solutions.}

The measures of numerical (blue), analytical (red), and analytical approximations of different orders (shades of gray with order numbers) are plotted as functions of numerical precision, where the dashed line indicates the most relevant for real-time simulation precision of $1 \mathrm{~ms}$. A. 
Full cycle error in the estimation of phase transition times using the $1 \%$ neighborhood of the optimal solution. Because the higher orders of approximations provide the same high precision as the cubic approximations, powers $T^{4}-T^{9}$ are not displayed. B. Similar to A, the errors are shown for the random distribution of parameters. C. Average CPU time needed to calculate a full step period of $1.25 \mathrm{~s}$ (average from Halbertsma's equations) in Python/NumPy implementation. The data presented in all subplots was averaged over $10^{5}$ trials.

Figure 4-3 shows the comparison of the processing cost between analytical and numerical solutions for this model (Equation 4-1). The analytical solution was the best choice for precise real-time applications of this model, outperforming the numerical method by close to an order of magnitude. However, if estimation errors of over $10 \mathrm{~ms}$ are insignificant in a specific application, e.g. using EMG-driven simulations with aggressive low-pass filtering, then high orders of analytical approximations could provide appropriate solutions with even lower computational load than the full analytical solution. The approximations of powers 3-9 use the eigenvalue approach to find roots of polynomials, which is relatively costly but still more precise than some of the comparable numerical integrators. The quadratic approximation (gray line marked 2) provided the lowest computational cost and similar quality to the analytical solution (red line), but only with the close-to-optimal parameter sets (the lowest $10 \% \operatorname{cost} J c$ as defined by Equation 4-4).

\section{Parametric sensitivity}

A perturbation analysis was used to investigate the parametric sensitivity of suboptimal solutions that satisfy Equation 4-4. This analysis compared optimal values found by several different local minimization methods after a 10\% normal parametric perturbation (for details, see steps five and six in section "Optimization and perturbation" of Methods). From 160 solutions, the $33 \%$ with the lowest Jc were: 30 by COBYLA, 1 by L-BFGS-B, 22 by Powell's algorithm, and 2 by Truncated Newton's. COBYLA and Powell's algorithms provided 95\% of the best solutions in this problem. The distribution of parameters in Figure 4-4 with similar cost (JC) across all methods indicates that similar outputs could be produced with disparate circuit parameters. The parameters in the model were differently conserved across similar solutions: the input weights $\left(G_{u}\right)$ had lower variability relative to other parameters, i.e. the static leak $\left(x_{0}\right)$, static input $\left(u_{0}\right)$, and interlimb connection weights (green, $r_{i j}$ ). 

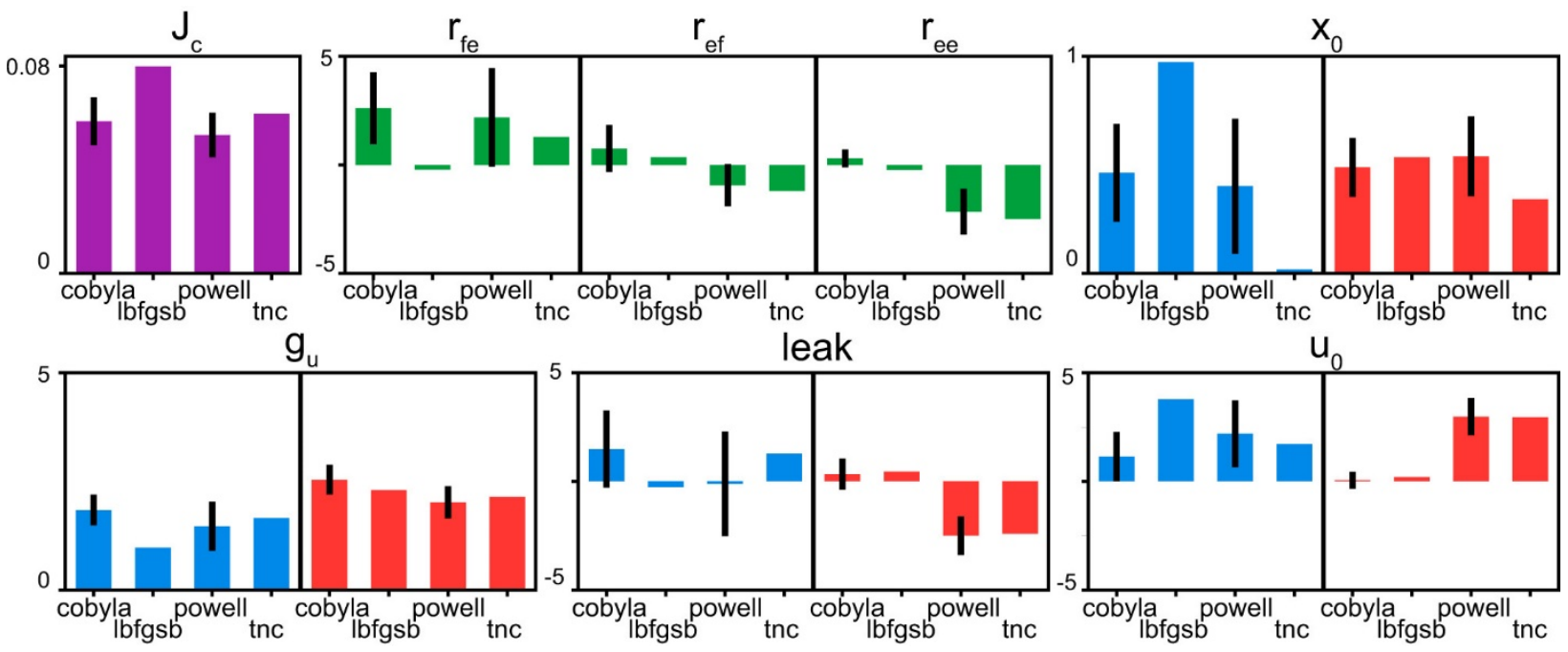

Figure 4-4. Analysis of parameter sensitivity.

The distributions of model parameters and cost function $(J c)$ are shown for the selection of best optimization sets. Each subplot shows a mean with standard deviation of the parameter values in blue (flexor), red (extensor), and green (mixed) for 4 types of minimization algorithms. The vertical axis range reflects the full feasible range of parameters as determined by the examination of intermediate solutions (see step six in section "Optimization and parameter perturbation" of Methods), with the exception for the Jc values.

\section{Behavioral implications of CPG morphology}

The velocity hypothesis states that descending signals to a CPG are the desired speeds of each leg. We wanted to test further if the analytical solution to the ODEs would produce the same or a different speed prediction for the modality of inputs. The direct relationship between the descending input and the temporal characteristics of stepping (step cycle, swing, and stance durations) was extracted from the second-order solution to Equation 4-3. Although it has a complex non-linear form (Equation 4-7), its combination with the solution from Goslow et al. (1973) for the relationship between step cycle period and forward speed produced a linear result shown in Figure 4-5 $\left(r^{2}=0.999, p<0.001\right.$ for left and right limbs). 


\section{Equation 4-7}

$$
V=\left(\frac{k_{1}+k_{2} u}{k_{3}-\sqrt{k_{4}+k_{5} u}}+k_{6}\right)^{1.68}
$$

where $k_{i}$ are configuration-dependent constants, $u$ is descending input, and $V$ is the forward speed of locomotion.

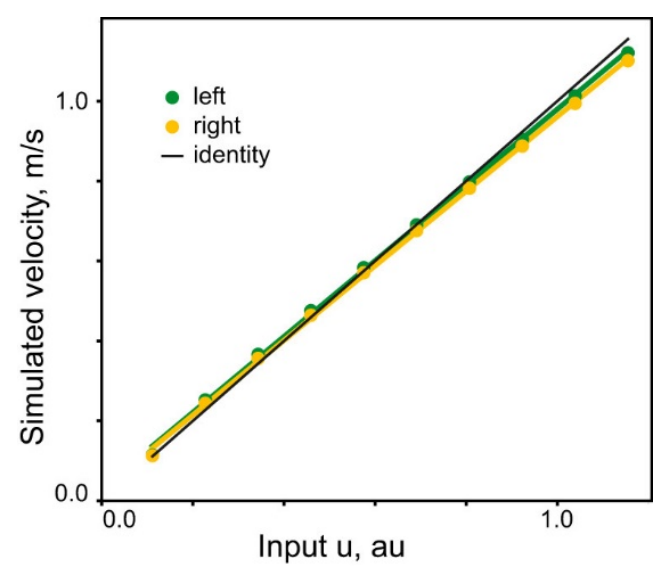

Figure 4-5. The relationship between the simulated CPG command signal to each limb and forward velocity.

The analytical solution for the full step cycle was calculated over the set of 10 input values for each limb $(u)$. Each value produced simulated step cycle duration values, which was then plotted as forward velocity calculated with the experimental relationship from Goslow et al. (1973) for each limb. The identity $(y=x)$ is plotted in black.

We further explored the role of this descending command for velocity regulation in the generation of asymmetric gait. Asymmetric patterns were simulated by uncoupling the gains for the left and right inputs of both flexors and extensors $\left(g_{u f 1}, g_{u e 1}, g_{u f 2}, g_{u e 2}\right)$ in Equation 4-2 and varying them independently by $33 \%$ of the optimal parameter set (Table 1 ). The $\mathrm{C}$ and $\mathrm{M}$ components responsible for pattern symmetricity and simulated speed related errors were removed from the cost function (Equation 4-4) in this analysis. The simulated speed of walking for the left and right limbs was then calculated from the generated bilateral phases (Figure 4-6). 
The parameter asymmetricity led to a steady gradient of the speed differences ( $\alpha=V_{\text {leff }} / V_{\text {right }}$, see Methods).
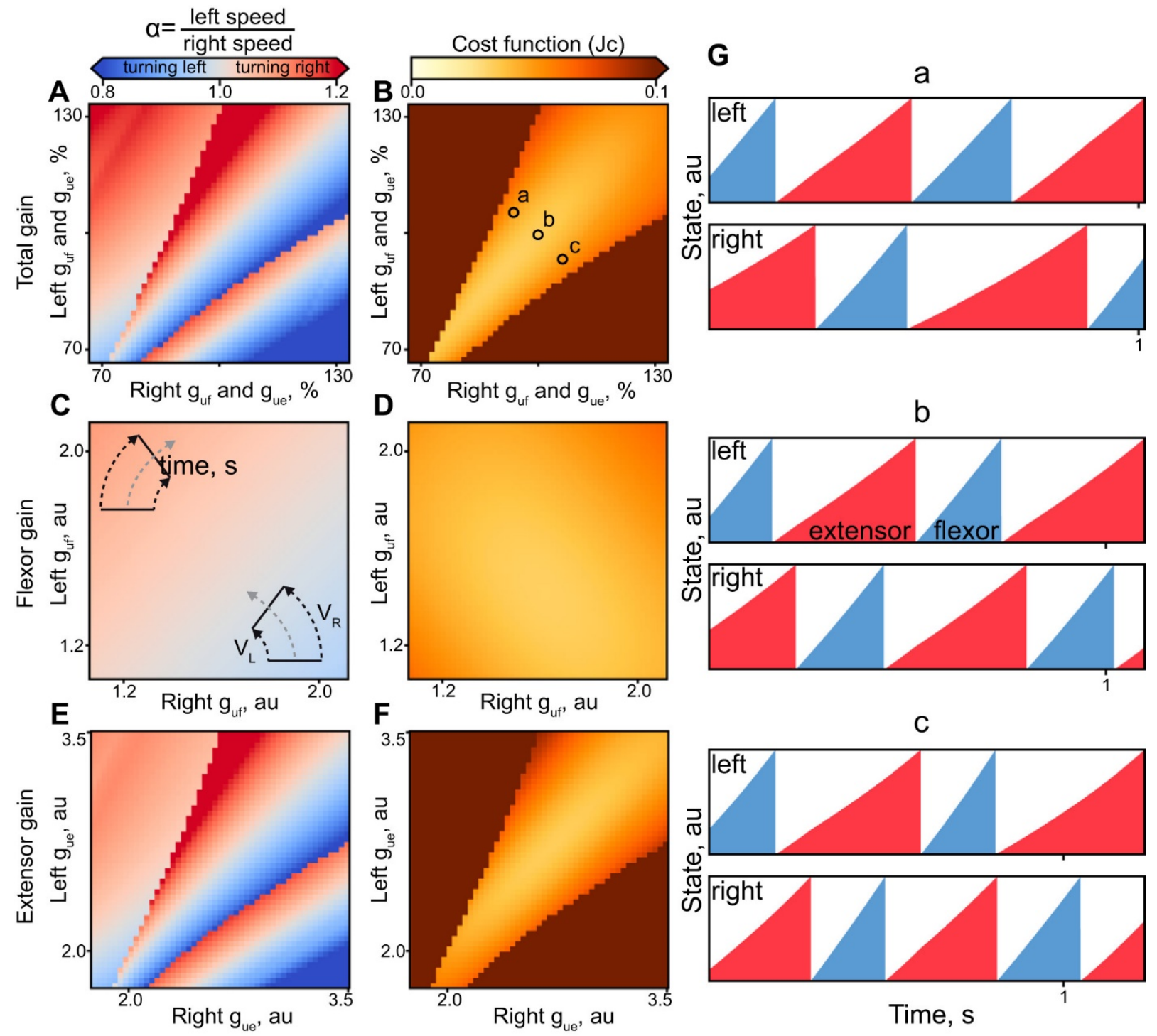

Time, s

Figure 4-6. External inputs generate asymmetric gait in the model.

The coupled and uncoupled input gain parameters $\left(g_{u e}, g_{u f}\right)$ were related to the velocity asymmetry (Left panels) with the corresponding cost function outputs (Right panels). A and B. The input gains of flexors and extensors were varied together for each limb. C and D. Only flexor input gains (left and right $g_{u f}$ ) were manipulated for each limb. E and F. Only extensor input gains (left and right $g_{u e}$ ) were manipulated for each limb. Inserts in C indicate the steering 
direction for two selected parameter sets. G. 'Raw' data showing the integration rates with simulation parameters $(a, b, c)$ selected in $\mathbf{B}$.

Table 1. Optimal model parameters. The parameter set $\left(z^{*}\right)$ for Equation $4-2$ that satisfies Equation 4-4.

\begin{tabular}{l|l|l|l|l|l|l|l|l|l|l}
$\mathrm{x}_{\mathrm{Of}}$ & $\mathrm{x}_{\mathrm{Oe}}$ & $\mathrm{g}_{\mathrm{uf}}$ & $\mathrm{g}_{\mathrm{ue}}$ & $\mathrm{g}_{\mathrm{lf}}$ & $\mathrm{g}_{\mathrm{le}}$ & $\mathrm{u}_{\mathrm{of}}$ & $\mathrm{u}_{0 \mathrm{e}}$ & $\mathrm{r}_{\mathrm{fe}}$ & $\mathrm{r}_{\mathrm{ef}}$ & $\mathrm{r}_{\mathrm{ee}}$ \\
\hline 0.244 & 0.376 & 1.59 & 2.62 & -0.689 & 0.828 & 2.26 & -0.174 & -0.025 & 2.38 & 0.418
\end{tabular}

Figure 4-6A and B show that variation of both inputs $\left(g_{u f}, g_{u e}\right)$ together can produce asymmetric walking, $\alpha=1.1$, with the turn diameter as low as $10 \mathrm{~m}$ (calculated from Equation 4-5, or heading direction $\gamma=10^{\circ}$ change per step, see Equation 4-6). Only the parameter combinations corresponding to the continuous gradient around the midline produced appropriately accurate simulations with low Jc (Figure 4-6B). Uncoupled inputs to flexors and extensors can similarly generate asymmetric gaits, with $\alpha$ up to $1.2\left(\gamma=20^{\circ}\right)$. The gradient of cost for extensors was orthogonal to that for flexors in Figure 4-6D and F; the increased possible range of asymmetric speeds was associated with increased cost, as indicated in Figure 4-6B, with the cost trough extending along the diagonal unity.

Figure 4-7 shows that the intrinsic parameters in the model can also produce asymmetric gaits. Symmetric connections (e.g. in Equation 4-2, $\left.r_{f e}=r_{14}=r_{41}\right)$ were uncoupled $\left(r_{14} \neq r_{41}\right)$ and varied independently. As in the analysis above, $\alpha$ and $J c$ were calculated for parameter variations of up to $\pm 33 \%$ of the optimal value. The connections from flexor to contralateral extensor did not provide a suitable gradient of asymmetric walking speeds in the explored range of parameters (Figure 4-7A). Possible reasons are a low magnitude of the optimal value for this parameter $\left(r_{\text {ef, }}\right.$ in Table 1$)$ and the near constant relationship between swing duration and locomotor speed (Figure 4-2). The variation of extensor-to-flexor and extensor-to-extensor parameters $\left(r_{\mathrm{ef}}, r_{\mathrm{ee}}\right)$ produced asymmetric gaits (Figure 4-7C and E) with a turn diameter of $10 \mathrm{~m}$ (heading direction $\gamma=10^{\circ}$ per step). These were comparable to the above result obtained from the analysis of external inputs. The profile of $J c$ was different for the gaits generated by variation of $r_{e e}$ and $r_{e f}$ parameters (Figure 4-7D and F). The extensor-to-flexor parameter $r_{e f}$ increased 
steering angle with a smaller increase in cost (Figure 4-7F) than that of the extensor-to-extensor parameter, $r_{e e}$ (Figure 4-7D). However, $r_{e e}$ could regulate asymmetric gaits over a larger range of velocities than $r_{\mathrm{ef}}$, as indicated by the diagonally extending trough in the cost function in Figure 4-7F.
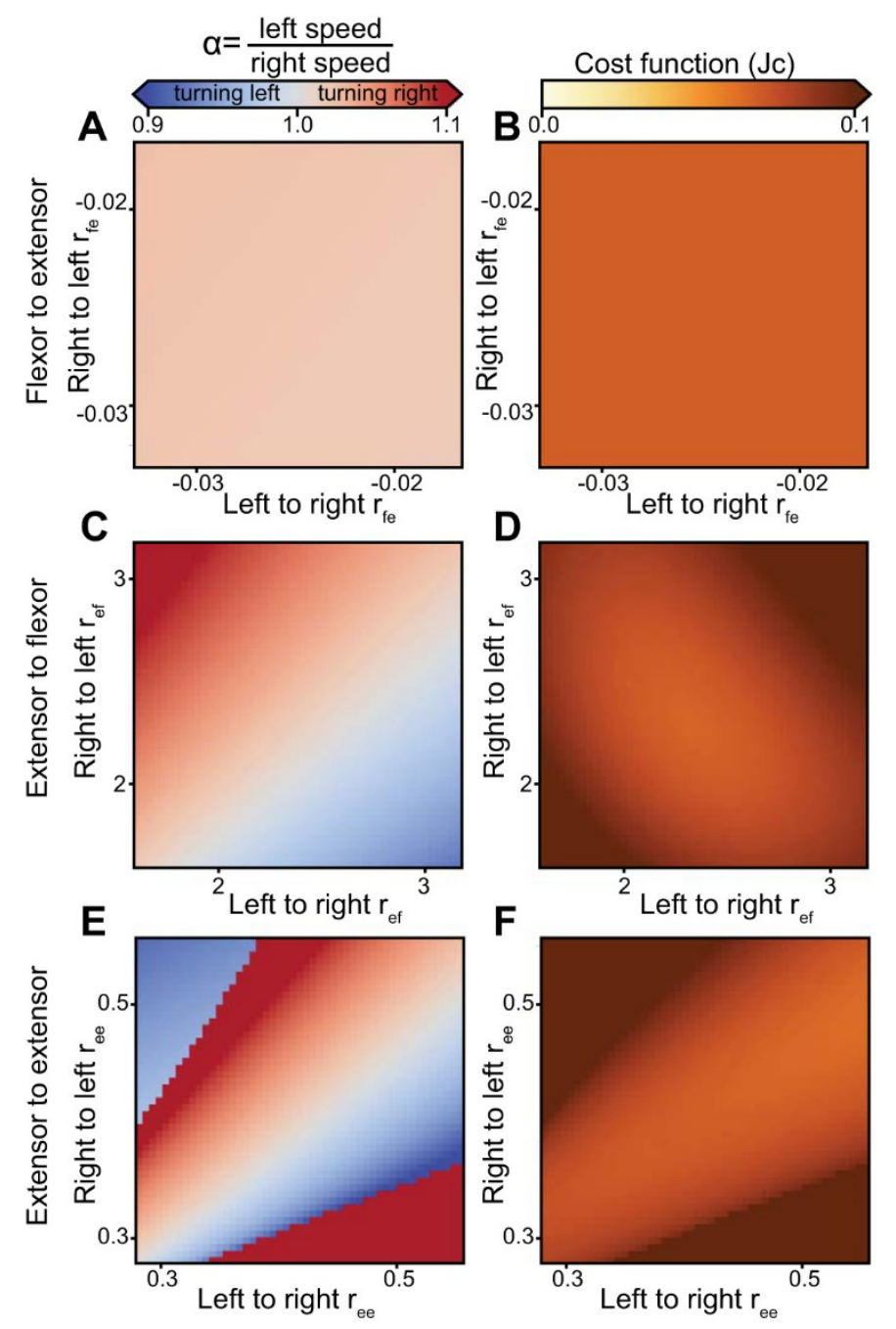

Figure 4-7. Intrinsic parameters generate asymmetric gait in the model.

The uncoupled intrinsic parameters $\left(r_{f e}, r_{e f}, r_{e e}\right)$ were related to the velocity asymmetry (Left panels) with the corresponding cost function outputs (Right panels). A and B. The flexor-toextensor weights $\left(r_{f e}\right)$. C and D. The extensor-to-flexor weights $\left(r_{e f}\right)$. E and F. The extensor-toextensor weights $\left(r_{e e}\right)$. 


\section{Discussion}

In this study, we developed a novel analytical description of a simple CPG model for locomotor phase timing and further expanded our previous model (Yakovenko et al., 2005) to include not only externally-driven asymmetric rhythmogenesis but also the opportunity to internalize this asymmetric transformation within the structure of a CPG. Our three central results are: i) the model can be solved analytically; ii) the analytical solution converges on the same conclusion that the input to the CPG is in the modality of limb forward speed; and iii) the minimalistic model of a CPG built with coupled oscillating leaky integrators offers multiple opportunities for embedding asymmetric control.

\section{What is the goal of using analytical solutions of neurophysiological models?}

Numerical solutions are usually the preferred option of solving complex models. For example, a biophysical CPG model can capture the neurological basis of activity in detail, often using hundreds of approximated parameters and their reconfiguration during failures in the motor execution (Rybak et al., 2006).

Complex models with multiple estimated transformations may produce ensemble behavior that reproduces the expected outcome; however, the role of elements and their network properties are hard to predict and analyze. The choice of a simple rate network with continuous variables in this model ignoring spike rate adaptation, dendritic processes, membrane dynamics, synaptic conductances, and plasticity has the advantage of focusing on the computation performed by the network connectivity (Abbott et al., 2016). Unlike models that are not analytically solvable, simple models are often insightful and capable of identifying specific targets that modify circuit behavior (Schaal and Sternad, 1998; Tabak et al., 2000; Izhikevich, 2004; Barnett and Cymbalyuk, 2014). For example, in the study of Barnett and Cymbalyuk (2014) two saddle node bifurcations, one for equilibria and one for periodic orbits, allowed to independently manipulate silent and spiking phase of bursting activity (Barnett and Cymbalyuk, 2014). The employed bifurcation control method relies on the manipulation of a controlling parameter near a transition between different regimes responsible for spiking and bursting properties. Spardy et al. (2011) showed how the dynamical system analysis could identify the silent and bursting periods of system's oscillation, the effect of sensory inputs on the range of 
behavior, and the operation of the CPG model in response to simulated spinal cord injury (Spardy et al., 2011a). This description was based on the simplified model (Markin et al., 2010; Spardy et al., 2011b) that uses two types of neuron implementations consisting of one- or twodimensional differential equations for a single limb flexor-extensor CPG. Similar to other much more complex implementations (Morris and Lecar, 1981; Rybak et al., 2006; Caplan et al., 2014), even this simplified formulation produces a challenging system of equations for 10 neurons with 33 connections between them. The model did noticeably have problems resolving locomotor phases for fast cycle durations (less than 800 ms, see Fig. 3 in Spardy et al., 2011b)). In contrast, our simple CPG model had only 4 parameters within a reciprocally connected system of 2 leaky integrators and simulated the same behavior without the aberrations at the extremes of experimental data (Yakovenko et al., 2005). This basic model that we extended in this study was used to describe, for the first time, the novel flexibility of extensor- and flexordominant phase regulation.

As in other models, we were concerned that expanding the model's parametric space to describe two limbs could introduce an uncontrollable increase in errors associated with the corresponding parametric expansion. The bilateral half-centers for two limbs required a system of 4 differential equations and the set of either 7 coupled (see Equation 4-2) or 16 uncoupled intrinsic and 4 extrinsic (input) parameters. The results for the expanded model in Figure 4-2 showing phase modulation over the full range of walking velocities without limitations at the extremes was not a forgone conclusion. Overall, the increased parametric complexity in the model did not lead to an overfitting problem that could have appeared from estimating too many parameters from a low-dimensional set of behavioral data. Instead, the model consistently converged on similar solutions without the loss of validity indicated by the cost function.

Overfitting and underfitting are two major concerns in the selection of appropriate levels of abstraction for models (Lever et al., 2016). In the words of John von Neumann, "With four parameters I can fit an elephant and with five I can make him wiggle his trunk." Here, our relatively simple model generates low-dimensional output in the form of the phase characteristic in normal and asymmetric locomotion. Models based on Hodgkin-Huxley formalism could generate the same phase duration characteristic, albeit with the use of large model parameter sets that extend into hundreds and thousands. Remarkably, the solutions from these two different representations are similar, supporting the experimental and computational observations that the same network activity could be generated by the underlying disparate 
mechanisms (Prinz et al., 2004; Goaillard et al., 2009; Grashow et al., 2009; Caplan et al., 2014). Still, the convergence of our parameter search on the physiological network solution is validated only by the constraining behavioral data and extent of simulated validation using parameter sensitivity analysis. Even in this minimalistic model, the exploration of a 20dimensional parameter space was challenging and led us to implement the analysis of a coupled symmetrical model first, where the parameters representing spinal neural elements mirrored across the midline were set to the same values. The perturbations in each parameter achieved with different minimization algorithms produced robust solutions, where small changes did not lead to large changes in outcome (Figure 4-4). Thus, the model may not be overfitting for these particular phenomena under study.

It is important to note that the model structure was not optimized in this study; instead, we have chosen the simplest mechanistic model of neural processing with structural elements identified by neurophysiology. Moreover, the quality of fit to the experimental data was very high ( $\mathrm{R}^{2}$ of about 0.999 ). The high $\mathrm{R}^{2}$ values are often used to support model validity. The typical additional validation is the analysis of residuals. The low values of residuals have no particular meaning when experimental data reconstruction is this precise. Then, the only possible failure of this model is the assumption that it is overly complicated or overfitting the experimental data, which is contradicted by the minimalistic design. This model has been already refined to the simplest mechanistic form.

\section{Embedding of asymmetric gait control in extrinsic and intrinsic parameters}

Even in our relatively simple model, there is a complicated relationship between intrinsic connections and extrinsic inputs. An indication of this fact is the capacity for representing the same behavior within parameters corresponding to different anatomical structures. Thus, it was necessary to uncouple the parameters in Equation 4-2 to further extend the sensitivity analysis with the goal of exploring the functionality "hidden" in the complexity to generate falsifiable hypotheses or model predictions.

We chose asymmetric gait as the test task because it results from the normal control of steering or heading direction (Yakovenko, 2011; Galbreath et al., 2014), and it may contain indicators of long-term adaptations to injury. First, we "forced" the model to internalize the control of asymmetric stepping by changing only extrinsic parameters. The mechanism using only input gains of flexor half-centers, and less so extensor half-centers, was a robust method of 
changing the interlimb speed differential. This was also expressed as a change in the heading direction in this model. In Figure 4-6, the tuning of input gains to flexor half-centers led to asymmetric speed ratios of 0.9 to 1.1 , which corresponds to an estimated heading direction change of $\pm 10^{\circ}$ over one step cycle (about a $10 \mathrm{~m}$ turn diameter). This suggests that a single external input representing a heading direction could generate a realistic range of asymmetric gaits in this model. Second, we can similarly constrain the solution to the locus of intrinsic parameters responsible for the influences among four half-centers in the model. It was intriguing to see the capability of this model to embed the asymmetric processing within these pathways. Moreover, the simulations suggested that not all parameters are equal targets in that respect. The extensor-to-flexor and extensor-to-extensor $\left(r_{e f}, r_{e e}\right.$ in Figure 4-7) parameters embedded the ability to generate asymmetric gaits with a reasonable turn diameter of $10 \mathrm{~m}$, which is consistent with a "step turning" strategy, characterized by a wide base of support throughout the turn. It is likely that steeper turning would require the transition to a different "spin turning" strategy (Hase and Stein, 1999; Taylor et al., 2005). The alternative CPG configurations are illustrated in the schematic in Figure 4-8. In studies of spinal segmental connectivity, these parameters would correspond to the 'gains' of propriospinal pathways connecting rhythmogenic networks within the spinal enlargement (Kiehn, 2011). Given the more rostral distribution of flexors than extensors within the lumbosacral enlargement (Yakovenko et al., 2002; Ivanenko et al., 2008) $r_{\text {ef }}$ and $r_{\text {ee }}$ pathways would have the network representations shown in Figure 4-8B and C. Overall, relatively complex behavior, like steering, could be controlled with both extrinsic and intrinsic mechanisms available in this simple model.

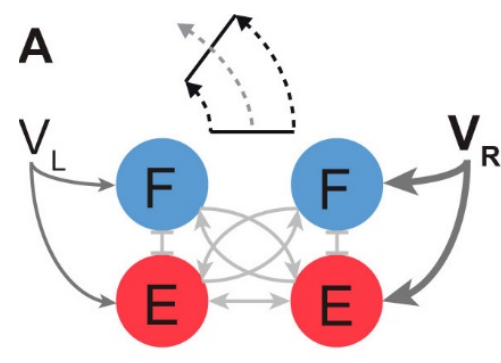

extrinsic

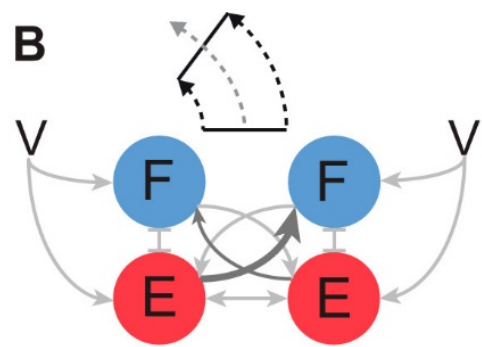

intrinsic ascending crossing

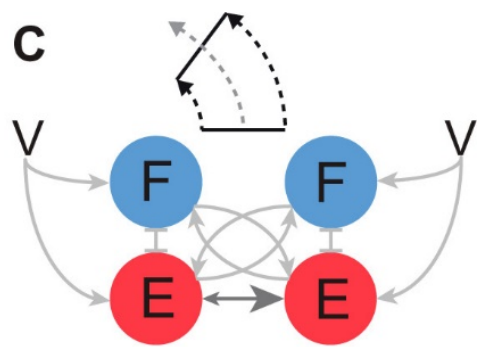

intrinsic crossing

Figure 4-8. Schematic representation of multiple CPG configurations for steering. 
A. The configuration based on the external inputs to CPG. B and C. Two possible configurations of intrinsic connections producing the same asymmetric patterns as in $\mathbf{A}$.

The analysis makes specific predictions about the propriospinal pathways that could be involved in long-term adaptations to asymmetricity. Human subjects could learn to compensate for the external perturbations applied to limbs while minimizing the overall limb impedance (Shadmehr and Mussa-Ivaldi, 1994; Dingwell et al., 2002). Even gross cortical inputs, like those generated by transcortical magnetic stimulation, can be compensated by the adaptation of transmission gains contributing to the regulation of locomotion (Schubert et al., 1999). Our results suggest that this adaptation can take place not only within pathways projecting to a CPG, but also within the limited locus of interactions between model's half-centers. While this model has no realistic learning dynamics, the examination was limited to the naïve symmetrical and adapted asymmetrical states. This learning function could be implemented in future work with the use of simple learning mechanisms (Franklin et al., 2008; Wu et al., 2014) where intrinsic and whole system parameters (ljspeert et al., 2013) could be updated under the reinforcement learning dynamics (Mahmoudi et al., 2013; Schultz, 2013).

Overall, the model demonstrated that the general locomotor patterns for symmetric and asymmetric gaits may be achieved by the superposition of commands and intrinsic interactions within the minimalistic structure of a CPG. This novel flexibility of functional representation for asymmetric pattern generation has not been previously demonstrated in models, and it posits specific predictions for mal- or adaptations to asymmetry due to peripheral or central abnormalities.

\section{The simple model of locomotor rhythm generation}

This model is not likely producing the overfitting of behavior as indicated by the sensitivity analysis. However, the excessive number of parameters relative to the number of observed values may hinder CPG models that do not address this methodological issue. Still, there is the alternative possibility that this model is instead underfitting the locomotor patterns associated with asymmetric gait. To discuss the appropriate level of abstraction that limits the possibility of underfitting for this task, we need to examine the concept of neuromechanical tuning (Prochazka and Yakovenko, 2007; Ting et al., 2015). Specifically, locomotor control is a 
phenomenon produced by multiple elements that combine predictive and reactive functions. In analogy with the Smith's predictor (Smith, 1957), the specific role of the CPG is to predict the mechanical interactions between the limb and ground. To this extent, our model can reproduce the transformation from input speeds to appropriate inter- and intra-limb coordination of multiple muscle groups without the need for molecular level dynamics (Yakovenko, 2011). The CPG function could then be specified as a dynamical transformation of simple, i.e., related to limb speed, high-level signals into complex granular functional subdivisions of temporal activations appropriate for locomotion. Both analytical and numerical solutions of our minimalistic CPG model support the hypothesis that the main function of a CPG is the transformation of high-level locomotor signals associated with whole limb function, i.e. the speed of locomotion, into lowlevel phasic activity patterns of limb muscles. This computational inference agrees with previous studies demonstrating that the one-dimensional input to the MLR in the form of stimulation magnitude or frequency can be transformed by a CPG into specific velocity-dependent phasic activity in vertebrates (Shik et al., 1966; Smetana et al., 2010). The underfitting for CPG models describing the phase duration characteristic would be classified by the inability to use high-level signals related to the forward velocity as the control signal for asymmetric gait. We demonstrated that this model can readily transform limb velocity-related inputs into asymmetric phase characteristics. Moreover, the model can embed these high-level representations within its internal structure. As shown previously (Yakovenko et al., 2005), it can also generate both flexor-dominated and extensor-dominated phase regulation at different speeds.

The model supports the idea that the CPG inputs are limb speeds. The positive relationship between the excitatory input and the frequency of network output oscillations has been previously modeled in CPG models using Hodgkin-Huxley formalism (Shevtsova et al., 2015; Danner et al., 2016). In contrast, our model does not assume the shape and relationship of CPG input with limb speed; this linear relationship was demonstrated by solving inversely the calculation performed by the structure proposed by T.G. Brown with the assumption of leaky integration (Yakovenko, 2011). This model was first introduced to describe the atypical flexor dominated pattern in fictive locomotion induced by the stimulation of midbrain locomotor region (MLR) and exhibiting the increased slope of phase vs. cycle duration for flexors rather than extensors (Yakovenko et al., 2005). The network state could be explained by the possible shift in the balance of descending drives that lack the extensor-biased limb load feedback and may have increased flexor biased inputs from MLR, e.g., see Fig. 5 in Frigon et al. (Frigon and Gossard, 2009), where MLR stimulation increases the duration of flexor bursts. 
The regulation of velocity-related control signals within supraspinal pathways remains to be poorly understood. The presence of spinocerebellar loops contributing to the modulation of vestibulospinal neurons (Arshavsky et al., 1978; Arshavsky and Orlovsky, 2016) warrants a theoretical re-examination of these contributions in the context of limb speed control. Cerebellum output to the motor cortex updates limb and body state to modulate the statedependent activity of its output, pyramidal tract neurons, and also receives inputs from networks in posterior parietal cortex responsible for planning and converting visual information (Drew and Marigold, 2015). The modulated discharge of neurons shows tuning to many velocity-related parameters during locomotion and reaching movements (Yakovenko and Drew, 2015). Theoretically, the velocity command can be extracted by the integration of dynamical neural discharge, or it may be represented in the activity of subpopulations of neurons with static discharge profiles. This leads to a complete closed-loop view of the steering control achieved by multiple supraspinal networks contributing to the transformation from optical flow to the control of heading direction (Warren et al., 2001) with the use of limb speed inputs to CPG, described in this study.

To conclude, in this paper we report for the first time a model of bilateral CPG with analytical and numerical solutions capable of generating symmetrical and asymmetrical gaits appropriate for whole body steering. The steering behavior can be generated by either extrinsic limb velocity related inputs to left and right half-center oscillators or embedded asymmetry within intrinsic propriospinal gains from extensor half-centers to the contralateral flexor or extensor halfcenters. Moreover, these asymmetric changes may correspond to either a natural control of limb velocity adjustments regulating the heading direction or pathological changes to the inputs or structure of the locomotor CPG. The existence of multiple network states capable of generating the same empirical observations is a novel identified challenge for CPG models.

\section{Appendix}

\section{Analytical solution}

The bilateral CPG model produces flexor (swing) and extensor (stance) phases for two limbs in relation to extrinsic input and intrinsic structure. To obtain these phases, Equation 4-1 needs to be integrated in time between the state changes. Numerical integration was previously used (Yakovenko, 2011) to generate swing and stance periods. The same transition points can be calculated analytically by transforming Equation 4-1 into a matrix Cauchy problem: 


\section{Equation 4-8}

$$
\left\{\begin{array}{c}
\dot{x}-A x=B \\
x(t=0)=x_{0}
\end{array}\right.
$$

where $A=G_{l}+G$ represents the intrinsic structure of the CPG, $B=U_{0}+G_{u} u$ represents the stateindependent inputs, and $x_{0}$ is the initial condition. In the case of a non-singular matrix $A$, this system has a vector form solution:

\section{Equation 4-9}

$$
x=A^{-1}\left(e^{A t}-I\right) B+e^{A t} x_{0}
$$

where $I$ is the identity matrix. This analytical expression of states $x$ (with dimensionality [4×1] for a model of bilateral CPG) describes the progression of all locomotor phases in time between the state changes. The remaining task is then to calculate the transition times and corresponding phase durations for a full step cycle. Equation 4-9 was evaluated for all three possible bilateral combinations of concurrent flexor-extensor activity during a full step cycle, namely: i) left flexion and right extension (states $x_{1}$ and $x_{4}$ ), ii) left extension and right extension (states $x_{2}$ and $x_{4}$ ), and iii) left extension and right flexion (states $x_{2}$ and $x_{3}$ ). States may have repeated more than once within the step cycle, when CPG activity was highly asymmetric. The dimensionality of the problem can be reduced from 4 to 2 because only two integrators are active at any given time with the following parameters:

\section{Equation 4-10}

$$
A=\left(\begin{array}{ll}
a_{i i} & a_{i j} \\
a_{j i} & a_{j j}
\end{array}\right), A^{-1}=\left(\begin{array}{cc}
\tilde{a}_{i i} & \tilde{a}_{i j} \\
\tilde{a}_{j i} & \tilde{a}_{j j}
\end{array}\right), B=\left(\begin{array}{l}
b_{i} \\
b_{j}
\end{array}\right), x_{0}=\left(\begin{array}{l}
x_{0 i} \\
x_{0 j}
\end{array}\right)
$$

where $i \in\{1,2\}$ and $j \in\{3,4\}$ are the indices of the two active integrators. We can then find the time of phase transitions $\tau$ for a given integrator $k$ by inserting the reduced parameter set (Equation 4-10) into Equation 4-9 and assuming $x_{i}$ or $x_{j}$ is equal to 1. Solving for $T$ yields the following transcendental equation:

\section{Equation 4-11}

$$
z_{1} \cdot \cosh (q \tau)+z_{2} \cdot \frac{\sinh (q \tau)}{q}=z_{3} \cdot e^{-s \tau}
$$

where $z_{1}, z_{2}, z_{3}, s, q$ are parameters describing the model configuration, as follows: 


$$
\begin{gathered}
s=\frac{a_{i i}+a_{j j}}{2} \\
q=\sqrt{\left(s-a_{j j}\right)^{2}+a_{i j} \cdot a_{j i}} \\
z_{1}=d_{1}+d_{4} \\
z_{2}=\left(s-a_{j j}\right) \cdot d_{1}+a_{i j} \cdot d_{2}+a_{j i} \cdot d_{3}-\left(s-a_{j j}\right) \cdot d_{4}
\end{gathered}
$$

Four-element vector $\mathrm{D}=\left[d_{1}, d_{2}, d_{3}, d_{4}\right]$ and $z_{3}$ depend on whether the model is solved for $i$ (left) limb: $D=\left(\tilde{a}_{i i} \cdot b_{i}+x_{0 i} ; \tilde{a}_{i i} \cdot b_{j}+x_{0 j} ; \tilde{a}_{i j} \cdot b_{i} ; \tilde{a}_{i j} \cdot b_{j}\right), z_{3}=1+\tilde{a}_{i i} \cdot b_{i}+\tilde{a}_{i j} \cdot b_{j}$, or $j$ (right) limb: $D=\left(\tilde{a}_{j i} \cdot b_{i} ; \tilde{a}_{j i} \cdot b_{j} ; \tilde{a}_{j j} \cdot b_{i}+x_{0 i} ; \tilde{a}_{j j} \cdot b_{j}+x_{0 j}\right), z_{3}=1+\tilde{a}_{j i} \cdot b_{i}+\tilde{a}_{j j} \cdot b_{j}$.

$T$ was then found numerically using Brent's method and analytically by expanding the hyperbolic functions using a Maclaurin series. We used the NumPy 'roots' function (Horn and Johnson, 2012) to solve the polynomials of power over 2. Next, the periods of activity of flexors and extensors during a step cycle were obtained with the following iterative algorithm:

i. $\quad$ Calculate the time $\tau_{i}$ when state $x_{i}$ reaches 1.

ii. Calculate the time $\tau_{j}$ when state $x_{j}$ reaches 1.

iii. Calculate the state of all integrators at time point $T=\min \left(T_{i}, T_{j}\right)$.

iv. Reset the state from 1 to 0 , deactivate it, and activate the reciprocal ipsilateral state. For example, switch from an active left flexor to an active left extensor.

v. If a full step cycle is completed (all 4 states reached value 1 at least once), stop; otherwise, go to step (i).

The code implementing the algorithm and parameters in Python is available on https://bitbucket.org/nishbo/cpg.

\section{Simulations with non-optimal parameters}

The model can generate a rich variety of patterns using parameters shaped by the cost function. The illustration of these insightful 'outtakes' is shown in Figure 4-9. The omission of the cost function components responsible for the cycle duration range (Figure 4-9A) results in the trivial best-fit solution that fits the desired phase relationship, but it may not reflect the range of phases with the increased magnitude of inputs $\left(G_{u}=0\right)$. Similarly, removing the cost of 
unphysiological bilateral flexor coactivation may result in the simulations that resemble hopping behavior (Figure 4-9B). The rapid switching in the heading direction can be simulated by forcing the system to cross the heading direction transition shown in Figure 4-9C\&D between points $C$ and $d$ in the panel $G$ from Figure 4-6. In this region, the asymmetrical change in $G_{u}$ for flexors and extensors flips the speed of left and right limbs as indicated by the difference of stance phase (dashed lines). The drop in the cost function of parameters in $d$ was associated largely with the $\mathrm{H}$ component of Equation 4-4 $(H c=0.05, H d=0.2)$. Selecting the parameters at the extremes (see $e$ and $f$ in Figure 4-9G) shows the examples of reduced coupling within the network. The patterns could drift relative to each other as in Figure 4-9F. The double-stepping, a physiological behavior (see Fig. 5 in Yakovenko, 2011), could also be generated by the large difference in the input to flexors and extensors. 

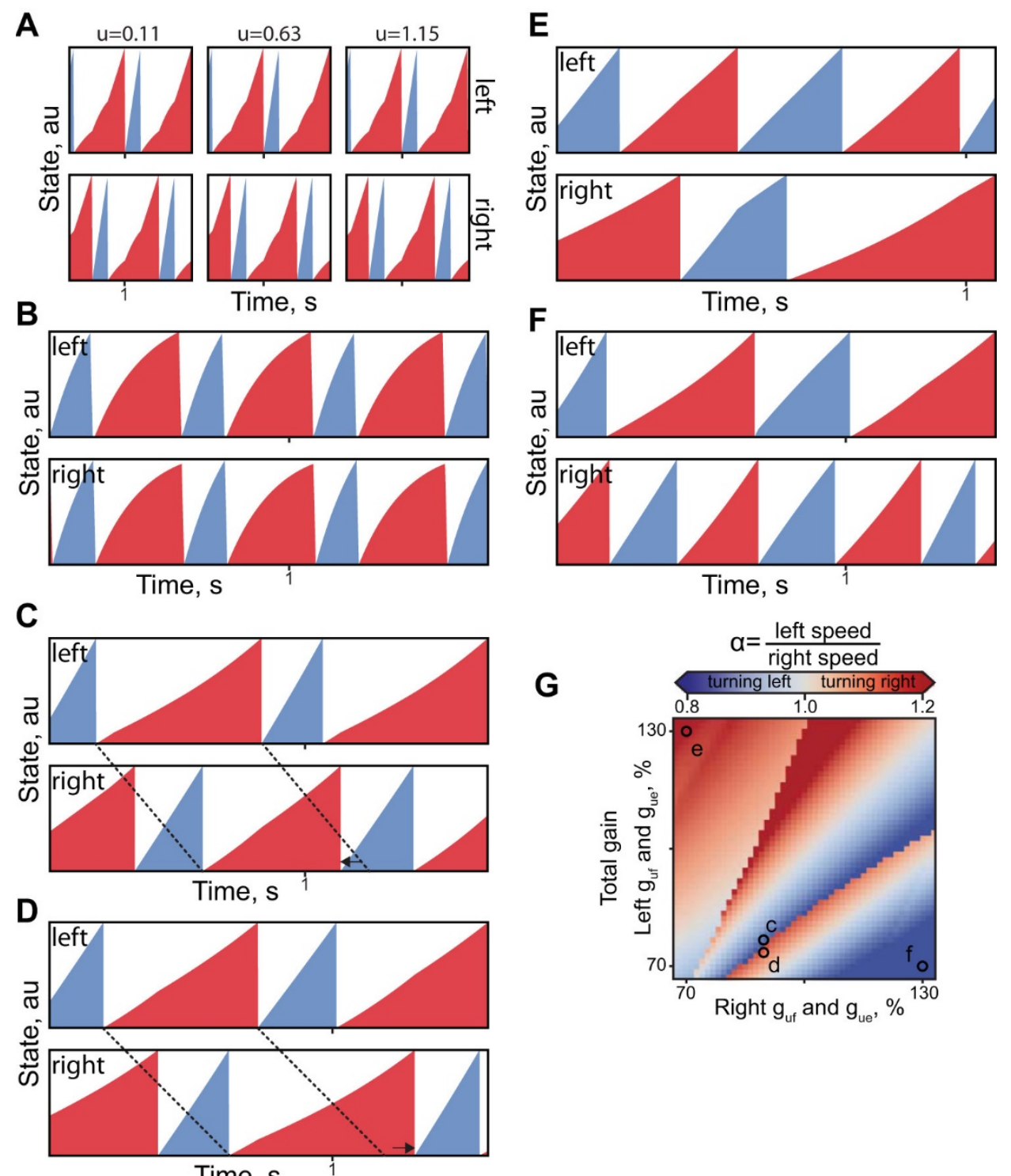

Time, $\mathrm{s}$

Figure 4-9. Solutions with the varied manipulations of the optimization algorithm.

A. Examples of patterns optimized without the range cost $\left(M, k_{2}=0\right.$ in Equation 4-4). B. Simulation without the cost of bilateral flexor activation $\left(O, k_{3}=0\right.$ in Equation 4-4). C and D. Simulations with uncoupled asymmetrical Gu parameters selected on the different sides of the heading direction transition (points $c$ and $d$ in $\mathbf{G}$ ). E and F. Simulations with uncoupled asymmetrical $G_{u}$ in points $e$ and $f$ of panel $\mathbf{G}$. The panel of Figure 4-6A with the indication of selected regions. 


\section{Acknowledgements}

The authors thank Dr. Jonathan Rubin for the general discussion of analytical solutions in locomotor CPG modeling. We thank the West Virginia Clinical and Translational Science Institute for editorial support. 


\section{Chapter 5 Discussion and Future Directions}

The dissertation explored motor control mechanisms at the levels of musculoskeletal actuators, their command signals and controlling networks utilizing an inverse model approach. The second chapter described a novel autogenerating model of kinematic MS variable approximations. The optimal polynomials showed very low computational demand and allowed detailed modelling of the human hand in real time. Structures of the polynomial models embedded functional and anatomical properties of the muscles. The third chapter utilizes the models developed in chapter two to provide inverse solutions to the neuromechanical control of the hand. Muscle excitation profiles from the datasets of desired postural and movement kinematics were obtained with regulated joint stiffness. Computed muscle excitations (CMEs) reproduced the desired movements and posture-maintaining torques with low errors. The agonist-antagonist coactivation mechanism partially explained the produced joint stiffness. A method of using the inverse solutions in prosthesis control was demonstrated and applied offline for a subject. Going further upstream in motor control, the fourth chapter focused on the phasic group motoneuron activity generated by spinal central pattern generators (CPGs) for locomotion. We showed how this model of the CPG is driven by the control signal in the form of desired forward velocity. The parameter space of the CPG model allowing symmetric and asymmetric walking was explored. We found several strategies for smooth turning potentially employed by the spinal cord and supraspinal control networks. Chapters two and three were prepared as a stand-alone peer-reviewed articles, to be submitted for review in a journal, and chapter four was published in a peer-reviewed journal.

In summary, three main chapters explored the mechanisms behind the generation of movement from pattern generation network activity to motor unit activation to biomechanics. Each of the chapters two, three and four presented a discussion specific to their respective topic. Several themes that span two or more of the chapters of this dissertation are addressed below, including: (i) mechanistic and phenomenological approaches in modelling; (ii) specifics of the mathematical spaces of that contain the described data; (iii) subject-specific variability encompassed by the models; and (iv) methodological and algorithmical specifics. The segments discuss current state, limitations, and future directions. 


\section{Mechanistic and Phenomenological Models}

Models employed in this dissertation utilized both mechanistic and phenomenological approaches. Approximations of the MS kinematic variables were fully phenomenological reflecting the goal of compact descriptions of simulated muscle path (Delp et al., 2007). We found that known functional and anatomical grouping was embedded within their structure. This embedded information in the form of polynomial components has the potential to be used as a descriptive language for MS structures.

Inverse solutions to the forward model of motor control simulated the behavior of the system. The presented forward and inverse models were a simulated analytical description of the motor control, describing all transformations directly or with reasonable approximations. As a part of the solution, a linear pseudoinverse had to be obtained, which is commonly considered to be a phenomenological approach (Mauk, 2000). Compared to common machine learning approaches (Ciancio et al., 2016; Nieveen et al., 2017; Resnik et al., 2018), the analytical representations enabled by this model allowed the investigation of the separate components of the control scheme contributing to the behavior of a complex model, i.e. the analyzed joint stiffness.

The CPG model also approximated very complex dynamics of control present in the spinal cord. Both simulation and analytical versions of the model were able to predict and produce a range of behaviors: symmetrical and asymmetrical locomotion, and compensation strategies (Sobinov and Yakovenko, 2017; Yakovenko et al., 2018). Its analysis revealed that the relatively simple CPG model (compared to neuron simulating approaches as in Rybak et al., 2006) has complex internal dynamics and can have multiple solutions producing the same patterns of control. Complex models describing the same phenomena need to be very carefully constructed and verified to make sure that they are representing the phenomena and internal dynamics correctly, otherwise they might be susceptible to unexpected, unstable behaviors, and wrong predictions. Using both analytical and phenomenological approaches in our model development helped ensure the correctness of their behavior and made them transparent.

\section{Mathematical Spaces}

The models implied the continuousness and sometimes smoothness of the spaces they were applied to. It is a commonly taken assumption for the description of MS variables, because the 
opposite would mean teleportation of the muscle between two paths absent in muscle mechanics (Menegaldo et al., 2004; Delp et al., 2007; Sartori et al., 2012). If such a behavior were to be observed in our data, it meant that there was an error in the OpenSim simulation, leading to a muscle falling off of a wrapping surface. Applying the approximation algorithm to other datasets with known discontinuities (e.g. transitions between phases) may require adding discontinuous functions or their approximations to the list of basis functions (See below in Methods and Algorithms). Another common implicit assumption for MS system is that there is only one anatomical path that a muscle travels through when a subject assumes a specific posture. Mathematically, it means that there is only one set of kinematic variables corresponding to each posture. If there is more than one set of variables in a posture, the muscle can be in multiple locations while the body is in a given posture. There is a theoretical possibility of that happening, for example, when the muscle path depends on how the body got to that posture, but I have failed to find any literature describing such behavior. In another theoretical example a muscle displaces other muscles that wrap around it or its tendons when it is flexed. Modelling of that behavior would require a very detailed representation of soft tissue biomechanics and has not yet been developed.

Control signals within the motor system are commonly assumed to be continuous in the form of single-neuron spike rate or population average activity (see for example Hochberg et al., 2006; Churchland et al., 2012). From a different point of view, precise single spikes encode more information (Strong et al., 1998; Reinagel and Reid, 2000) and have been found as a primary mechanism in specific motor tasks, like songbird breathing (Srivastava et al., 2017). The precise timing of neuron synchronization similar to the observed discontinuous single-spike encoding can also be simulated in a continuous neuron behavior (Izhikevich, 2001). The debate is still ongoing, and our observed smoothness of the computed muscle excitation profiles is a product of the continuousness of the kinematics and internal model components. It is important to note the contribution of the smoothness of the model components to the smoothness of observed behavior, because when non-smooth relationships between MS variables and force were used in the model, I observed oscillations in forward control and discontinuous profiles in the inverse. Both of these effects were detrimental for the stable control. From that I suggest ensuring the smoothness of the relationships for stable MS model behavior, especially for prosthetic controllers. 
On the higher level of control, spinal CPGs produce state transitions from walking to trot, to running when the stimulation of brainstem MLR region increases (Shik et al., 1966). Such discontinuous output with state transitions was outside of the scope of the analysis of my dissertation, which focused on walking, albeit the model has capability to support other gaits (Yakovenko, 2011). Many CPG models support state transition as an emergent property of the continuous neuronal dynamics (Danner et al., 2017). Analyzing the model configurations corresponding to different gaits is an intriguing direction for the future research that can answer some of the questions about the size of the parameter space posed in my research. Other than rare examples, discontinuities in motor control are not commonly observed and can be modelled by smooth and continuous models.

\section{Subject-Specific Variability}

While intersubject variability is a common problem for prosthetic controllers, it was mostly outside of the focus of my dissertation. Many of the developed models represented the first step in the development of a model at their scale and attempted to describe the average behavior of the system. In the field of MS modelling, validity of the mean description is currently debated (Akita and Nimura, 2016a, 2016b; Goislard De Monsabert et al., 2018; Boots et al., 2019). During our analysis, we found that modelling the average MS system by combining different sources of measurements was viable, and sometimes the only available way, considering the barrenness of the available data. The modelled MS system that we obtained is a useful first approximation in which segments can be scaled to better represent morphometrics of a specific subject.

Variability of subject-specific control signals is one of the biggest problems for prosthetic controllers (Hiremath et al., 2015). In machine learning approaches it leads to long individualized training periods (Cipriani et al., 2011; Nieveen et al., 2017). In a biomimetic control scheme it leads to manual or automated tweaking of model parameters (Crouch and Huang, 2016). An inverse solution of the neuromechanical system accommodates subjectspecific variation in control of biomimetic prosthetics by scaling and redistribution of subject's EMG signals into the control signals via a regression between EMGs and CMEs, which theoretically are in the same domain. A big limitation of the current approach that potentially hindered the ability of the inverse to identically replicate the desired traces when controlled by a subject's EMG, was the static and generic values for joint stiffness. In the current application, influence of stiffness was limited to an engineering improvement on the stability of the controlled 
system. Even if the relation between the MS model and the MS system of the subject through CMEs would not be perfectly established, a subject should be able to learn how to operate a slightly different modelled body, as happens in motor adaptation (Krakauer and Mazzoni, 2011).

CPGs, especially describing the rhythmogenic control, as the model described in Chapter 4, are a conservative neural structure in mammalian CNS (Kandel et al., 2000). The synergies activated by the rhythm vary in different species, often corresponding to specifics of MS structure (Oliveira et al., 2014). In all described cases, an average representative behavior is needed to build more complex models than a single subject data would allow.

Some of the limitations of the models in their representation of subject specificity can be addressed by future developments. The MS model can incorporate scaling of segments to produce kinematic variables specific for a subject (Goislard de Monsabert et al., 2017; Pan et al., 2018). A potential problem with that approach is the computational errors of muscle path estimation similar to slipping off of a wrapping surface that need to be investigated. A way to bypass this problem is a transformation routine in the space of optimal approximating polynomials that would allow obtaining differently-scaled models without the need to verify the MS simulation or re-optimize the polynomials. The optimal polynomials from different subjects can be used to refine our understanding of functional and anatomical clusters similar to the analysis performed in Chapter 2. If applied to different species, it can be used in evolutionary biology to quantitatively track the anatomical and neuromuscular changes happening in species and their functional implications. To be feasible, such an extensive analysis would first require an automated method for building an MS model from some imaging data (Blemker et al., 2007), and its application to an extensive dataset. As mentioned previously, the purpose of the inverse solution to the model of hand motor control was to negate the subject-specific and recordingspecific variability. Further progress can be made by extracting the stiffness values from a movement. The additional part of the cost function on the regression for the whole or part of the dataset will encourage low residuals in the regression between CMEs and EMGs. This would allow a more precise estimation of the user's intent and account for the unknown stiffness present in human movements. Fitting of the CPG model parameters to the user-specific behavior can be used in a medical setting to assess motor disabilities or dangerous behaviors of a subject. For example, by finding asymmetricities and identifying their source, a strategy can be developed to change gait to reduce fall risk and improve safety. Investigating the estimation 
of stiffness in the motor commands from the recorded data and its use in prosthetic control will be an area for future research.

\section{Methods and Algorithms}

The methods and algorithms described in this dissertation can be further improved to account for other datasets and phenomena. Algorithms used to approximate the dataset of MS kinematic variables can be expanded to include other basis functions, e.g. trigonometric, exponential, discontinuous or generalized, to accommodate the specifics of the desired dataset. Such expansion is supported by the algorithm structure and has been used during development, although in the case of discontinuous basis functions, another regression algorithm may be needed for parameter estimation. A covariation matrix can be used to guide the selection of this increased pool of basis functions. There is a theoretical possibility of the algorithm stopping the approximation prematurely, when it would need to add two terms at a time to improve the information criterion. To investigate if it was influencing the approximation of the MS dataset, I have implemented a version of the algorithm, which, when finished with approximation, attempted to find an addition of two or more polynomial terms that improved the information criterion. If they were added, the optimization was resumed. The optimal polynomials did not change when obtained using said algorithm, but the time required to obtain the functions increased to several days for 6-DOF muscles.

An important future direction of the present research would be to evaluate the different relationships between EMGs and CMEs in the modelled hand control using inverse solutions. As an example, we considered: (i) using generalized linear regression; (ii) relating the changes in EMGs (first time derivative) to CMEs, (iii) relating independent components of EMGs to CMEs, or (iv) using an artificial neural network. Comparison of these approaches will require a formalized test and a dedicated set of experiments with multiple subjects under similar conditions.

An improvement of the CPG parameter estimation algorithm can include an explicit constraint on the full step periodicity of the analytical CPG model. These are commonly omitted as a constraint in experimental studies (Thelen and Anderson, 2006), but might provide an insight into different parameters balancing each other. I think it would be fruitful to pursue these directions of research in the future, expanding the approximation algorithm and implementing other relationships between CMEs and EMGs on an organized dataset. 
Overall, my dissertation described formation of movements within motor control systems. At the spinal level, rhythmogenesis in the spinal cord CPGs was analyzed from the intent encoded in the supraspinal regions. An internal model was proposed for estimation of motor unit excitation from the desired movements. These motor commands were executed through a complex biomechanical model of the hand. All these parts fit together to produce a description of motor control by the central nervous system. Future developments based on the fundamental models and their principles describe herein will hopefully lead to the development of prosthetic devices that will help restore motor function of people and improve their quality of life. 


\section{References}

Abbott LF, DePasquale B, Memmesheimer R-M (2016) Building functional networks of spiking model neurons. Nat Neurosci 19:350-355.

Abboudi RL, Glass CA, Newby NA, Flint JA, Craelius W (1999) A biomimetic controller for a multifinger prosthesis. IEEE Trans Rehabil Eng 7:121-129.

Akaike H (1974) A new look at the statistical model identification. IEEE Trans Automat Contr 19:716-723.

Akita K, Nimura A (2016a) Forearm Muscles. In: Bergman's Comprehensive Encyclopedia of Human Anatomic Variation, pp 298-314. Hoboken, NJ, USA: John Wiley \& Sons, Inc.

Akita K, Nimura A (2016b) Arm Muscles. In: Bergman's Comprehensive Encyclopedia of Human Anatomic Variation, pp 293-297. Hoboken, NJ, USA: John Wiley \& Sons, Inc.

Alexander GE, Crutcher MD (1990) Functional architecture of basal ganglia circuits: neural substrates of parallel processing. Trends Neurosci 13:266-271.

Alexander RM (1997) A minimum energy cost hypothesis for human arm trajectories. Biol Cybern 76:97-105.

Alon G, Levitt AF, McCarthy PA (2007) Functional electrical stimulation enhancement of upper extremity functional recovery during stroke rehabilitation: a pilot study. Neurorehabil Neural Repair 21:207-215.

Ambike S, Mattos D, Zatsiorsky VM, Latash ML (2016) Synergies in the space of control variables within the equilibrium-point hypothesis. Neuroscience 315:150-161.

An KN, Takahashi K, Harrigan TP, Chao EY (1984) Determination of muscle orientations and moment arms. J Biomech Eng 106:280-282.

Angel RW (1974) Electromyography during voluntary movement: the two-burst pattern. Electroencephalogr Clin Neurophysiol 36:493-498.

Antony NT, Keir PJ (2010) Effects of posture, movement and hand load on shoulder muscle activity. J Electromyogr Kinesiol 20:191-198.

Arshavsky YI, Gelfand IM, Orlovsky GN, Pavlova GA (1978) Messages conveyed by descending tracts during scratching in the cat. I. Activity of vestibulospinal neurons. Brain Res 159:99-110.

Arshavsky YI, Orlovsky GN (2016) Role of the Cerebellum in the Control of Rhythmic Movements. In: Neurobiology of Vertebrate Locomotion.

Barnett WH, Cymbalyuk GS (2014) A codimension-2 bifurcation controlling endogenous bursting activity and pulse-triggered responses of a neuron model. PLoS One 9.

Bashor DP (1998) A large-scale model of some spinal reflex circuits. Biol Cybern 78:147-157.

Beggs WD, Howarth Cl (1972) The movement of the hand towards a target. Q J Exp Psychol 24:448-453.

Belter JT, Dollar AM (2011) Performance characteristics of anthropomorphic prosthetic hands. 
IEEE Int Conf Rehabil Robot.

Bendel RB, Afifi AA (1977) Comparison of stopping rules in forward "stepwise" regression. J Am Stat Assoc.

Berger DJ, d'Avella A (2014) Effective force control by muscle synergies. Front Comput Neurosci 8:1-13.

Berniker M, Jarc A, Bizzi E, Tresch MC (2009) Simplified and effective motor control based on muscle synergies to exploit musculoskeletal dynamics. Proc Natl Acad Sci U S A 106:7601-7606.

Bernstein NA (1967) The co-ordination and regulation of movements: Conclusions towards the Study of Motor Co-ordination. Biodyn Locomot:104-113.

Bhushan N, Shadmehr R (1999) Computational nature of human adaptive control during learning of reaching movements in force fields. Biol Cybern 81:39-60.

Blana D, Chadwick EK, van den Bogert AJ, Murray WM (2017) Real-time simulation of hand motion for prosthesis control. Comput Methods Biomech Biomed Engin 20:540-549.

Blemker SS, Asakawa DS, Gold GE, Delp SL (2007) Image-based musculoskeletal modeling: applications, advances, and future opportunities. J Magn Reson Imaging 25:441-451.

Blum KP, Lamotte D'Incamps B, Zytnicki D, Ting LH (2017) Force encoding in muscle spindles during stretch of passive muscle. Ayers J, ed. PLoS Comput Biol 13:e1005767.

Bonnot A, Whelan PJ, Mentis GZ, O'Donovan MJ (2002) Spatiotemporal pattern of motoneuron activation in the rostral lumbar and the sacral segments during locomotor-like activity in the neonatal mouse spinal cord. J Neurosci 22:RC203.

Boots M, Sobinov A, Gritsenko V, Gaunt RA, Yakovenko S (2019) Realistic musculoskeletal models for real-time human-in-the-loop control of arm and hand. (unpublished).

Brand PW, Cranor KC, Ellis JC (1975) Tendon and pulleys at the metacarpophalangeal joint of a finger. J Bone Joint Surg Am 57:779-784.

Brown TG (1911) The Intrinsic Factors in the Act of Progression in the Mammal. Proc R Soc B Biol Sci 84:308-319.

Burnham KP, Anderson DR (2004) Model Selection and Multimodel Inference (Burnham KP, Anderson DR, eds). New York, NY: Springer New York.

Byrd RH, Hribar ME, Nocedal J (1999) An Interior Point Algorithm for Large-Scale Nonlinear Programming. SIAM J Optim 9:877-900.

Byrd RH, Lu P, Nocedal J, Zhu C (1995) A Limited Memory Algorithm for Bound Constrained Optimization. SIAM J Sci Comput 16:1190-1208.

Cabelguen J-M, Bourcier-Lucas C, Dubuc R (2003) Bimodal locomotion elicited by electrical stimulation of the midbrain in the salamander Notophthalmus viridescens. J Neurosci 23:2434-2439.

Caplan JS, Williams AH, Marder E (2014) Many parameter sets in a multicompartment model oscillator are robust to temperature perturbations. J Neurosci 34:4963-4975. 
Cappellari P, Gaunt R, Beringer C, Mansouri M, Novelli M (2018) Identifying Electromyography Sensor Placement using Dense Neural Networks. :130-141.

Chadwick EK, Blana D, van den Bogert AJ, Kirsch RF (2009) A real-time, 3-D musculoskeletal model for dynamic simulation of arm movements. IEEE Trans Biomed Eng 56:941-948.

Churchland MM, Cunningham JP, Kaufman MT, Foster JD, Nuyujukian P, Ryu SI, Shenoy K V. (2012) Neural population dynamics during reaching. Nature.

Ciancio AL, Cordella F, Barone R, Romeo RA, Bellingegni AD, Sacchetti R, Davalli A, Pino G Di, Ranieri F, Lazzaro V Di, Guglielmelli E, Zollo L (2016) Control of prosthetic hands via the peripheral nervous system. Front Neurosci 10:1-17.

Cipriani C, Antfolk C, Controzzi M, Lundborg G, Rosen B, Carrozza MC, Sebelius F (2011) Online myoelectric control of a dexterous hand prosthesis by transradial amputees. IEEE Trans Neural Syst Rehabil Eng 19:260-270.

Conover WJ (1999) Chapter 3.4: The Sign Test. In: Practical Nonparametric Statistics (Third ed.), pp 157-176. Wiley.

Crouch DL, Huang H (2016) Lumped-parameter electromyogram-driven musculoskeletal hand model: A potential platform for real-time prosthesis control. J Biomech 49:3901-3907.

Cymbalyuk GS, Gaudry Q, Masino M a, Calabrese RL (2002) Bursting in leech heart interneurons: cell-autonomous and network-based mechanisms. J Neurosci 22:1058010592.

D'Agostino R, Pearson ES (1973) Tests for departure from normality. Empirical results for the distributions of $B$ and $\sqrt{ } b$. Biometrika.

d'Avella A, Portone A, Fernandez L, Lacquaniti F (2006) Control of fast-reaching movements by muscle synergy combinations. J Neurosci 26:7791-7810.

Danner SM, Shevtsova NA, Frigon A, Rybak IA (2017) Computational modeling of spinal circuits controlling limb coordination and gaits in quadrupeds. Elife 6:1-25.

Danner SM, Wilshin SD, Shevtsova NA, Rybak IA (2016) Central control of interlimb coordination and speed-dependent gait expression in quadrupeds. J Physiol 594:69476967.

De Serres SJ, Milner TE (1991) Wrist muscle activation patterns and stiffness associated with stable and unstable mechanical loads. Exp brain Res 86:451-458.

Deeny S, Chicoine C, Hargrove L, Parrish T, Jayaraman A (2014) A simple ERP method for quantitative analysis of cognitive workload in myoelectric prosthesis control and humanmachine interaction. PLoS One 9.

Delp SL, Anderson FC, Arnold AS, Loan P, Habib A, John CT, Guendelman E, Thelen DG (2007) OpenSim: Open source to create and analyze dynamic simulations of movement. IEEE Trans Biomed Eng 54:1940-1950.

Dietz V (2003) Spinal cord pattern generators for locomotion. Clin Neurophysiol 114:13791389.

Dingwell JB, Mah CD, Mussa-Ivaldi FA (2002) Manipulating objects with internal degrees of 
freedom: evidence for model-based control. J Neurophysiol 88:222-235.

Diogo R, Linde-Medina M, Abdala V, Ashley-Ross MA (2013) New, puzzling insights from comparative myological studies on the old and unsolved forelimb/hindlimb enigma. Biol Rev Camb Philos Soc 88:196-214.

Donald LB (1973) Biomechanics of the Shoulder Joint. Arch Surg 107:425-432.

Donelan JM, Kram R, Kuo AD (2001) Mechanical and metabolic determinants of the preferred step width in human walking. Proceedings Biol Sci 268:1985-1992.

Downey JE, Brane L, Gaunt RA, Tyler-Kabara EC, Boninger ML, Collinger JL (2017) Motor cortical activity changes during neuroprosthetic-controlled object interaction. Sci Rep 7:16947.

Drew T, Marigold DS (2015) Taking the next step: Cortical contributions to the control of locomotion. Curr Opin Neurobiol 33:25-33.

Durandau G, Farina D, Sartori M (2018) Robust Real-Time Musculoskeletal Modeling Driven by Electromyograms. IEEE Trans Biomed Eng 65:556-564.

Eilenberg MF, Geyer H, Herr H (2010) Control of a powered ankle-foot prosthesis based on a neuromuscular model. IEEE Trans Neural Syst Rehabil Eng 18:164-173.

Englehart K, Hudgins B (2003) A robust, real-time control scheme for multifunction myoelectric control. IEEE Trans Biomed Eng 50:848-854.

Flash T, Hogan N (1985) The coordination of arm movements: an experimentally confirmed mathematical model. J Neurosci 5:1688-1703.

Flesher SN, Collinger JL, Foldes ST, Weiss JM, Downey JE, Tyler-Kabara EC, Bensmaia SJ, Schwartz AB, Boninger ML, Gaunt RA (2016) Intracortical microstimulation of human somatosensory cortex. Sci Transl Med 8:361ra141.

Formento E, Minassian K, Wagner F, Mignardot JB, Le Goff-Mignardot CG, Rowald A, Bloch J, Micera S, Capogrosso M, Courtine G (2018) Electrical spinal cord stimulation must preserve proprioception to enable locomotion in humans with spinal cord injury. Nat Neurosci 21:1728-1741.

Franklin DW, Burdet E, Peng Tee K, Osu R, Chew C-M, Milner TE, Kawato M (2008) CNS Learns Stable, Accurate, and Efficient Movements Using a Simple Algorithm. J Neurosci 28:11165-11173.

Frigon A, Gossard J-P (2009) Asymmetric control of cycle period by the spinal locomotor rhythm generator in the adult cat. J Physiol 587:4617-4628.

Frigon A, Thibaudier Y, Hurteau M-F (2015) Modulation of forelimb and hindlimb muscle activity during quadrupedal tied-belt and split-belt locomotion in intact cats. Neuroscience 290:266-278.

Full RJ, Koditschek DE (1999) Templates and anchors: neuromechanical hypotheses of legged locomotion on land. J Exp Biol 202:3325-3332.

Galbreath K, Olesh E, Yakovenko S (2014) Do humans use limb velocity signal to control locomotion? Soc Neurosci. 
Georgopoulos AP, Kalaska JF, Massey JT (1981) Spatial trajectories and reaction times of aimed movements: effects of practice, uncertainty, and change in target location. $\mathrm{J}$ Neurophysiol 46:725-743.

Geyer H, Herr H (2010) A muscle-reflex model that encodes principles of legged mechanics produces human walking dynamics and muscle activities. IEEE Trans Neural Syst Rehabil Eng 18:263-273.

Gillard DM, Yakovenko S, Cameron T, Prochazka A (2000) Isometric muscle length-tension curves do not predict angle-torque curves of human wrist in continuous active movements. J Biomech 33:1341-1348.

Goaillard J-M, Taylor AL, Schulz DJ, Marder E (2009) Functional consequences of animal-toanimal variation in circuit parameters. Nat Neurosci 12:1424-1430.

Goislard De Monsabert B, Edwards D, Shah D, Kedgley A (2018) Importance of Consistent Datasets in Musculoskeletal Modelling: A Study of the Hand and Wrist. Ann Biomed Eng 46:71-85.

Goislard de Monsabert B, Rao G, Gay A, Berton E, Vigouroux L (2017) A scaling method to individualise muscle force capacities in musculoskeletal models of the hand and wrist using isometric strength measurements. Med Biol Eng Comput 55:2227-2244.

Goslow GE, Reinking RM, Stuart DG (1973) The cat step cycle: hind limb joint angles and muscle lengths during unrestrained locomotion. J Morphol 141:1-41.

Grashow R, Brookings T, Marder E (2009) Reliable neuromodulation from circuits with variable underlying structure. Proc Natl Acad Sci U S A 106:11742-11746.

Graupe D, Beex AA, Monlux WJ, Magnussen I (1977) A multifunctional prosthesis control system based on time series identification of EMG signals using microprocessors. Bull Prosthet Res.

Grillner S (1981) Control of locomotion in bipeds, tetrapods, and fish. Handb Physiol Nerv Syst II:1179-1236.

Grillner S, Wallén P (1985) Central pattern generators for locomotion, with special reference to vertebrates. Annu Rev Neurosci 8:233-261.

Grillner S, Wallen P, Saitoh K, Kozlov A, Robertson B (2008) Neural bases of goal-directed locomotion in vertebrates - An overview. Brain Res Rev 57:2-12.

Grillner S, Zangger P (1975) How detailed is the central pattern generation for locomotion? Brain Res 88:367-371.

Gritsenko V, Hardesty RL, Boots MT, Yakovenko S (2016) Biomechanical Constraints Underlying Motor Primitives Derived from the Musculoskeletal Anatomy of the Human Arm. PLoS One 11:e0164050.

Hägglund M, Dougherty KJ, Borgius L, Itohara S, Iwasato T, Kiehn O (2013) Optogenetic dissection reveals multiple rhythmogenic modules underlying locomotion. Proc Natl Acad Sci U S A 110:11589-11594.

Halbertsma JM (1983) the Stride Cycle of the Cat: By Computerized Analysis of Automatic Recordings. Acta Physiol Scand 521:4-521. 
Hallgrímsson B, Willmore K, Hall BK (2002) Canalization, developmental stability, and morphological integration in primate limbs. Am J Phys Anthropol Suppl 35:131-158.

Hase K, Stein RB (1999) Turning strategies during human walking. J Neurophysiol 81:29142922.

Hermann KM, Kočiský T, Grefenstette E, Espeholt L, Kay W, Suleyman M, Blunsom P (2015) Teaching Machines to Read and Comprehend. :1-9.

Hiremath S V., Chen W, Wang W, Foldes S, Yang Y, Tyler-Kabara EC, Collinger JL, Boninger ML (2015) Brain computer interface learning for systems based on electrocorticography and intracortical microelectrode arrays. Front Integr Neurosci 9:40.

Hochberg LR, Serruya MD, Friehs GM, Mukand JA, Saleh M, Caplan AH, Branner A, Chen D, Penn RD, Donoghue JP (2006) Neuronal ensemble control of prosthetic devices by a human with tetraplegia. Nature 442:164-171.

Hocherman S, Wise SP (1991) Effects of hand movement path on motor cortical activity in awake, behaving rhesus monkeys. Exp brain Res 83:285-302.

Hogan N (1984) Adaptive control of mechanical impedance by coactivation of antagonist muscles. IEEE Trans Automat Contr 29:681-690.

Hogan N (2002) Skeletal muscle impedance in the control of motor actions. J Mech Med Biol 02:359-373.

Holzbaur KRS, Murray WM, Delp SL (2005) A model of the upper extremity for simulating musculoskeletal surgery and analyzing neuromuscular control. Ann Biomed Eng 33:829840.

Hore J, Flament D (1988) Changes in motor cortex neural discharge associated with the development of cerebellar limb ataxia. J Neurophysiol 60:1285-1302.

Horn RA, Johnson CR (2012) Matrix Analysis. Cambridge University Press.

ljspeert AJ (2008) Central pattern generators for locomotion control in animals and robots: A review. Neural Networks 21:642-653.

Ijspeert AJ, Nakanishi J, Hoffmann H, Pastor P, Schaal S (2013) Dynamical movement primitives: learning attractor models for motor behaviors. Neural Comput 25:328-373.

Ison M, Artemiadis P (2014) The role of muscle synergies in myoelectric control: trends and challenges for simultaneous multifunction control. J Neural Eng 11:051001.

Ivanenko YP, Cappellini G, Poppele RE, Lacquaniti F (2008) Spatiotemporal organization of alpha-motoneuron activity in the human spinal cord during different gaits and gait transitions. Eur J Neurosci 27:3351-3368.

Ivanenko YP, Poppele RE, Lacquaniti F (2009) Distributed neural networks for controlling human locomotion: lessons from normal and SCI subjects. Brain Res Bull 78:13-21.

Izenman AJ (2008) Modern Multivariate Statistical Techniques.

Izhikevich EM (2001) Resonate-and-fire neurons. Neural Networks 14:883-894.

Izhikevich EM (2004) Which model to use for cortical spiking neurons? IEEE Trans Neural 
Networks 15:1063-1070.

Kandel ER, Schwartz JH, Jessell TM (2000) Principles of Neural Science.

Kaufman KR, An KN, Litchy WJ, Cooney WP, Chao EY (1999) In-vivo function of the thumb muscles. Clin Biomech (Bristol, Avon) 14:141-150.

Kawato M (1999) Internal models for motor control and trajectory planning. Curr Opin Neurobiol 9:718-727.

Kiehn O (2011) Development and functional organization of spinal locomotor circuits. Curr Opin Neurobiol 21:100-109.

Körding KP, Wolpert DM (2004) Bayesian integration in sensorimotor learning. Nature 427:244247.

Krakauer JW, Mazzoni P (2011) Human sensorimotor learning: adaptation, skill, and beyond. Curr Opin Neurobiol 21:636-644.

Kumar V, Todorov E (2015) MuJoCo HAPTIX: A virtual reality system for hand manipulation. In: 2015 IEEE-RAS 15th International Conference on Humanoid Robots (Humanoids), pp 657-663. IEEE.

Kuo AD (2002) The relative roles of feedforward and feedback in the control of rhythmic movements. Motor Control 6:129-145.

Lapuschkin S, Wäldchen S, Binder A, Montavon G, Samek W, Müller K-R (2019) Unmasking Clever Hans predictors and assessing what machines really learn. Nat Commun 10:1096.

Laurent K, De Sèze M-P, Delleci C, Koleck M, Dehail P, Orgogozo J-M, Mazaux J-M (2011) Assessment of quality of life in stroke patients with hemiplegia. Ann Phys Rehabil Med 54:376-390.

Lawrence JH, Nichols TR, English a W (1993) Cat hindlimb muscles exert substantial torques outside the sagittal plane. J Neurophysiol 69:282-285.

Leger AB, Milner TE (2000) Passive and active wrist joint stiffness following eccentric exercise. Eur J Appl Physiol 82:472-479.

Lever J, Krzywinski M, Altman N (2016) Points of Significance: Model selection and overfitting. Nat Methods.

Li ZM, Zatsiorsky VM, Latash ML (2000) Contribution of the extrinsic and intrinsic hand muscles to the moments in finger joints. Clin Biomech (Bristol, Avon) 15:203-211.

Lillicrap TP, Scott SH (2013) Preference Distributions of Primary Motor Cortex Neurons Reflect Control Solutions Optimized for Limb Biomechanics. Neuron 77:168-179.

Little S, Pogosyan A, Neal S, Zavala B, Zrinzo L, Hariz M, Foltynie T, Limousin P, Ashkan K, FitzGerald J, Green AL, Aziz TZ, Brown P (2013) Adaptive deep brain stimulation in advanced Parkinson disease. Ann Neurol 74:449-457.

Loren GJ, Shoemaker SD, Burkholder TJ, Jacobson MD, Fridén J, Lieber RL (1996) Human wrist motors: biomechanical design and application to tendon transfers. J Biomech 29:331342. 
Maganaris CN (2001) Force-length characteristics of in vivo human skeletal muscle. Acta Physiol Scand 172:279-285.

Mahmoudi B, Pohlmeyer EA, Prins NW, Geng S, Sanchez JC (2013) Towards autonomous neuroprosthetic control using Hebbian reinforcement learning. J Neural Eng 10:066005.

Mann HB, Whitney DR (1947) On a Test of Whether one of Two Random Variables is Stochastically Larger than the Other. Ann Math Stat.

Marder E, Calabrese RL (1996) Principles of rhythmic motor pattern generation. Physiol Rev 76:687-717.

Markin SN, Klishko AN, Shevtsova NA, Lemay MA, Prilutsky BI, Rybak IA (2010) Afferent control of locomotor CPG: Insights from a simple neuromechanical model. Ann N Y Acad Sci 1198:21-34.

Matsuoka K (1985) Sustained oscillations generated by mutually inhibiting neurons with adaptation. Biol Cybern 52:367-376.

Mauk MD (2000) The potential effectiveness of simulations versus phenomenological models. Nat Neurosci 3:649-651.

Menegaldo LL, de Toledo Fleury A, Weber HI (2004) Moment arms and musculotendon lengths estimation for a three-dimensional lower-limb model. J Biomech 37:1447-1453.

Morris C, Lecar H (1981) Voltage oscillations in the barnacle giant muscle fiber. Biophys J 35:193-213.

Nicolas-Alonso LF, Gomez-Gil J (2012) Brain computer interfaces, a review. Sensors (Basel) 12:1211-1279.

Nieveen J, Zhang Y, Wendelken S, Davis T, Kluger D, George JA, Warren D, Hutchinson D, Duncan C, Clark GA, Mathews VJ (2017) Polynomial Kalman filter for myoelectric prosthetics using efficient kernel ridge regression. Int IEEE/EMBS Conf Neural Eng NER:432-435.

Nocedal J, Wright S (2000) Numerical Optimization.

O'Donovan MJ, Bonnot A, Wenner P, Mentis GZ (2005) Calcium imaging of network function in the developing spinal cord. Cell Calcium 37:443-450.

Oliphant TE (2007) SciPy: Open source scientific tools for Python. Comput Sci Eng 9:10-20.

Oliveira AS, Gizzi L, Farina D, Kersting UG (2014) Motor modules of human locomotion: influence of EMG averaging, concatenation, and number of step cycles. Front Hum Neurosci 8:1-9.

Paclet F, Quaine F (2012) Motor control theories improve biomechanical model of the hand for finger pressing tasks. J Biomech 45:1246-1251.

Pan L, Crouch DL, Huang H (2018) Myoelectric Control Based on a Generic Musculoskeletal Model: Toward a Multi-User Neural-Machine Interface. IEEE Trans Neural Syst Rehabil Eng 26:1435-1442.

Pedregosa F, Varoquaux G, Gramfort A, Michel V, Thirion B, Grisel O, Blondel M, Prettenhofer P, Weiss R, Dubourg V, Vanderplas J, Passos A, Cournapeau D, Brucher M, Perrot M, 
Duchesnay E (2011) Scikit-learn: Machine Learning in Python. J Mach Learn Res.

Poggio T, Mhaskar H, Rosasco L, Miranda B, Liao Q (2017) Why and when can deep-but not shallow-networks avoid the curse of dimensionality: A review. Int J Autom Comput 14:503519.

Powell MJD (1964) An efficient method for finding the minimum of a function of several variables without calculating derivatives. Comput J 7:155-162.

Prinz AA, Bucher D, Marder E (2004) Similar network activity from disparate circuit parameters. Nat Neurosci 7:1345-1352.

Prochazka A, Ellaway P (2012) Sensory systems in the control of movement. Compr Physiol 2:2615-2627.

Prochazka A, Yakovenko S (2007) Predictive and reactive tuning of the locomotor CPG. Integr Comp Biol 47:474-481.

Quental C, Folgado J, Ambrósio J, Monteiro J (2015) Critical analysis of musculoskeletal modelling complexity in multibody biomechanical models of the upper limb. Comput Methods Biomech Biomed Engin 18:749-759.

Rajagopal A, Dembia CL, DeMers MS, Delp DD, Hicks JL, Delp SL (2016) Full-Body Musculoskeletal Model for Muscle-Driven Simulation of Human Gait. IEEE Trans Biomed Eng 63:2068-2079.

Rankin JW, Neptune RR (2012) Musculotendon lengths and moment arms for a threedimensional upper-extremity model. J Biomech 45:1739-1744.

Reinagel P, Reid RC (2000) Temporal coding of visual information in the thalamus. J Neurosci 20:5392-5400.

Resnik L, Huang HH, Winslow A, Crouch DL, Zhang F, Wolk N (2018) Evaluation of EMG pattern recognition for upper limb prosthesis control: a case study in comparison with direct myoelectric control. J Neuroeng Rehabil 15:23.

Rolian C, Lieberman DE, Hallgrímsson B (2010) The coevolution of human hands and feet. Evolution 64:1558-1568.

Rossi S, Rossini PM (2004) TMS in cognitive plasticity and the potential for rehabilitation. Trends Cogn Sci 8:273-279.

Rybak IA, Shevtsova NA, Lafreniere-Roula M, McCrea DA (2006) Modelling spinal circuitry involved in locomotor pattern generation: insights from deletions during fictive locomotion. $\mathrm{J}$ Physiol 577:617-639.

Sartori M, Reggiani M, van den Bogert AJ, Lloyd DG (2012) Estimation of musculotendon kinematics in large musculoskeletal models using multidimensional B-splines. J Biomech 45:595-601.

Schaal S, Sternad D (1998) Programmable pattern generators. 3rd Int Conf Comput Intell Neurosci.

Schiff ND, Giacino JT, Kalmar K, Victor JD, Baker K, Gerber M, Fritz B, Eisenberg B, Biondi T, O'Connor J, Kobylarz EJ, Farris S, Machado A, McCagg C, Plum F, Fins JJ, Rezai AR 
(2007) Behavioural improvements with thalamic stimulation after severe traumatic brain injury. Nature 448:600-603.

Scholz JP, Schöner G (1999) The uncontrolled manifold concept: identifying control variables for a functional task. Exp brain Res 126:289-306.

Schöner G, Jiang WY, Kelso JAS (1990) A synergetic theory of quadrupedal gaits and gait transitions. J Theor Biol 142:359-391.

Schubert M, Curt A, Colombo G, Berger W, Dietz V (1999) Voluntary control of human gait: conditioning of magnetically evoked motor responses in a precision stepping task. Exp brain Res 126:583-588.

Schultz W (2013) Updating dopamine reward signals. Curr Opin Neurobiol 23:229-238.

Scott RN, Parker PA (1988) Myoelectric prostheses: state of the art. J Med Eng Technol 12:143-151.

Scott SH, Kalaska JF (1995) Changes in motor cortex activity during reaching movements with similar hand paths but different arm postures. J Neurophysiol 73:2563-2567.

Shadmehr R, Mussa-Ivaldi F a (1994) Adaptive representation of dynamics during learning of a motor task. J Neurosci 14:3208-3224.

Shevtsova NA, Talpalar AE, Markin SN, Harris-Warrick RM, Kiehn O, Rybak IA (2015) Organization of left-right coordination of neuronal activity in the mammalian spinal cord: Insights from computational modelling. J Physiol 593:2403-2426.

Shik ML, Severin F V, Orlovskii GN (1966) [Control of walking and running by means of electric stimulation of the midbrain]. Biofizika 11:659-666.

Smetana R, Juvin L, Dubuc R, Alford S (2010) A parallel cholinergic brainstem pathway for enhancing locomotor drive. Nat Neurosci 13:731-738.

Smith OM (1957) Posicast Control of Damped Oscillatory Systems. Proc IRE 45:1249-1255.

Sobinov A, Boots M, Gritsenko V, Gaunt RA, Yakovenko S (2019) Functional description of musculoskeletal transformation using multidimensional polynomials. (unpublished).

Sobinov A, Yakovenko S (2017) Model of a bilateral Brown-type central pattern generator for symmetric and asymmetric locomotion. J Neurophysiol:jn.00443.2017.

Spardy LE, Markin SN, Shevtsova NA, Prilutsky BI, Rybak IA, Rubin JE (2011a) A dynamical systems analysis of afferent control in a neuromechanical model of locomotion: I. Rhythm generation. J Neural Eng 8:065003.

Spardy LE, Markin SN, Shevtsova NA, Prilutsky BI, Rybak IA, Rubin JE (2011b) A dynamical systems analysis of afferent control in a neuromechanical model of locomotion: II. Phase asymmetry. J Neural Eng 8:065004.

Sreenivasa M, Valero-Cuevas FJ, Tresch M, Nakamura Y, Schouten AC, Sartori M (2019) Editorial: Neuromechanics and Control of Physical Behavior: From Experimental and Computational Formulations to Bio-inspired Technologies. Front Comput Neurosci 13:13.

Srivastava KH, Holmes CM, Vellema M, Pack AR, Elemans CPH, Nemenman I, Sober SJ (2017) Motor control by precisely timed spike patterns. Proc Natl Acad Sci U S A 


\section{4:1171-1176.}

Stroeve S (1999) Impedance characteristics of a neuromusculoskeletal model of the human arm II. Movement control. Biol Cybern 81:495-504.

Strong SP, Koberle R, De Ruyter Van Steveninck RR, Bialek W (1998) Entropy and information in neural spike trains. Phys Rev Lett.

Sussillo D, Churchland MM, Kaufman MT, Shenoy K V (2015) A neural network that finds a naturalistic solution for the production of muscle activity. Nat Neurosci 18:1025-1033.

Tabak J, Senn W, O'Donovan MJ, Rinzel J (2000) Modeling of spontaneous activity in developing spinal cord using activity-dependent depression in an excitatory network. J Neurosci 20:3041-3056.

Taga G, Yamaguchi Y, Shimizu H (1991) Self-organized control of bipedal locomotion by neural oscillators in unpredictable environment. Biol Cybern 65:147-159.

Taylor MJD, Dabnichki P, Strike SC (2005) A three-dimensional biomechanical comparison between turning strategies during the stance phase of walking. Hum Mov Sci 24:558-573.

Terekhov A V., Zatsiorsky VM (2011) Analytical and numerical analysis of inverse optimization problems: conditions of uniqueness and computational methods. Biol Cybern 104:75-93.

Terekhov A V, Pesin YB, Niu X, Latash ML, Zatsiorsky VM (2010) An analytical approach to the problem of inverse optimization with additive objective functions: an application to human prehension. J Math Biol 61:423-453.

Thelen DG (2003) Adjustment of muscle mechanics model parameters to simulate dynamic contractions in older adults. J Biomech Eng 125:70-77.

Thelen DG, Anderson FC (2006) Using computed muscle control to generate forward dynamic simulations of human walking from experimental data. J Biomech 39:1107-1115.

Thorsen R, Spadone R, Ferrarin M (2001) A pilot study of myoelectrically controlled FES of upper extremity. IEEE Trans Neural Syst Rehabil Eng 9:161-168.

Ting LH (2007) Dimensional reduction in sensorimotor systems: a framework for understanding muscle coordination of posture. Prog Brain Res 165:299-321.

Ting LH, Chiel HJ, Trumbower RD, Allen JL, McKay JL, Hackney ME, Kesar TM (2015) Neuromechanical Principles Underlying Movement Modularity and Their Implications for Rehabilitation. Neuron 86:38-54.

Todorov E (2004) Optimality principles in sensorimotor control. Nat Neurosci 7:907-915.

Todorov E, Erez T, Tassa Y (2012) MuJoCo: A physics engine for model-based control. In: IEEE International Conference on Intelligent Robots and Systems, pp 5026-5033.

Todorov E, Jordan M (2002) Optimal feedback control as a theory of motor coordination. Nat Neurosci 5:1226-1235.

Tucker MR, Olivier J, Pagel A, Bleuler H, Bouri M, Lambercy O, Millán J del R, Riener R, Vallery $\mathrm{H}$, Gassert R (2015) Control strategies for active lower extremity prosthetics and orthotics: a review. J Neuroeng Rehabil 12:1. 
Valero-Cuevas FJ, Venkadesan M, Todorov E (2009) Structured Variability of Muscle Activations Supports the Minimal Intervention Principle of Motor Control. J Neurophysiol 102:59-68.

van der Krogt MM, de Graaf WW, Farley CT, Moritz CT, Richard Casius LJ, Bobbert MF (2009) Robust passive dynamics of the musculoskeletal system compensate for unexpected surface changes during human hopping. J Appl Physiol 107:801-808.

Verzár F (1923) Reflexumkehr (paradoxe Reflexe) durch zentrale Ermüdung beim Warmblüter. Pflugers Arch Gesamte Physiol Menschen Tiere 199:109-124.

Voisin JL (2006) Clavicle, a neglected bone: Morphology and relation to arm movements and shoulder architecture in primates. Anat Rec - Part A Discov Mol Cell Evol Biol 288:944953.

Wachholder K (1928) Willkiirliche Haltung und Bewegung insbesondere im Lichte electrophysiologischer Untersuchungen. Erg Physiol 26:568-775.

Wadman W, Denier van der Gon J, Geuze R, Mol C (1979) Control of fast goal-directed arm movements. J Hum Mov Stud 5:3-17.

Wagner FB et al. (2018) Targeted neurotechnology restores walking in humans with spinal cord injury. Nature 563:65-71.

Wales D, Doye JPK (1997) Global Optimization by Basin-Hopping and the Lowest Energy Structures of Lennard-Jones Clusters Containing up to 110 Atoms. J Phys Chem A 101:5111-5116.

Wallén P, Ekeberg O, Lansner a, Brodin L, Tråvén H, Grillner S (1992) A computer-based model for realistic simulations of neural networks. II. The segmental network generating locomotor rhythmicity in the lamprey. J Neurophysiol 68:1939-1950.

Warren WH, Kay BA, Zosh WD, Duchon AP, Sahuc S (2001) Optic flow is used to control human walking. Nat Neurosci 4:213-216.

Weston J, Chopra S, Bordes A (2014) Memory Networks. :1-15.

Winter DA (2009) Biomechanics and Motor Control of Human Movement. Hoboken, NJ, USA: John Wiley \& Sons, Inc.

Winters JM (1995) An improved muscle-reflex actuator for use in large-scale neuromusculoskeletal models. Ann Biomed Eng 23:359-374.

Wolpert DM, Ghahramani Z (2000) Computational principles of movement neuroscience. Nat Neurosci 3 Suppl:1212-1217.

Wolpert DM, Ghahramani Z, Jordan MI (1995) An internal model for sensorimotor integration. Science 269:1880-1882.

Wolpert DM, Miall RC, Kawato M (1998) Internal models in the cerebellum. Trends Cogn Sci 2:338-347.

Wu HG, Miyamoto YR, Gonzalez Castro LN, Ölveczky BP, Smith MA (2014) Temporal structure of motor variability is dynamically regulated and predicts motor learning ability. Nat Neurosci 17:312-321. 
Xu Z, Todorov E (2016) Design of a highly biomimetic anthropomorphic robotic hand towards artificial limb regeneration. Proc - IEEE Int Conf Robot Autom 2016-June:3485-3492.

Yakovenko S (2011) A hierarchical perspective on rhythm generation for locomotor control. Prog Brain Res 188:151-166.

Yakovenko S, Drew T (2015) Similar Motor Cortical Control Mechanisms for Precise Limb Control during Reaching and Locomotion. J Neurosci 35:14476-14490.

Yakovenko S, Gritsenko V, Prochazka A (2004) Contribution of stretch reflexes to locomotor control: a modeling study. Biol Cybern 90:146-155.

Yakovenko S, McCrea D a, Stecina K, Prochazka a (2005) Control of locomotor cycle durations. J Neurophysiol 94:1057-1065.

Yakovenko S, Mushahwar V, VanderHorst V, Holstege G, Prochazka A (2002) Spatiotemporal activation of lumbosacral motoneurons in the locomotor step cycle. J Neurophysiol 87:1542-1553.

Yakovenko S, Sobinov A, Gritsenko V (2018) Analytical CPG model driven by limb velocity input generates accurate temporal locomotor dynamics. PeerJ 6:e5849.

Zajac FE (1989) Muscle and tendon: properties, models, scaling, and application to biomechanics and motor control. Crit Rev Biomed Eng 17:359-411. 


\section{Curriculum Vitae}

\section{Contacts}

E-mail: an.sobinov@gmail.com

ORCID ID: orcid.org/0000-0003-0513-9194

Google Scholar: scholar.google.com/citations?user=CwogBilAAAAJ
Anton Sobinov

Personal data

Date of birth: April 14, 1992

Languages: Russian (native),

English (fluent)

\section{Education and Research Experience}

2014 - Present

Graduate student, PhD candidate in Neuroscience. Centers for Neuroscience, Department of Biomedical Sciences, West Virginia University, Morgantown, WV, USA.

2018, September-October

NSF Innovation Corps Course. Team STEVE, project: commercialization of augmented reality prosthesis.

2016, May 17-19

Summer School, IEEE SPS Summer School on Signal Processing and Machine Learning for Big Data, Pittsburgh, PA, USA.

$2013-2015$

Graduate student, M.Sc. in Applied Mathematics and Informatics, Department of Nano-, Bio-, Information and Cognitive Technologies, Moscow Institute of Physics and Technology (State University), Moscow, Russia.

Qualification: Applied Mathematics and Informatics.

GPA: 5.0 out of 5.0, cum laude.

Thesis: "Search for traces of Tataro-Mongolian admixture in genotypes of Eurasian nations". Grade: 5 out of 5 .

2014, January - June

Shader developer, Akra browser-based game engine (ODServe), Moscow, Russia.

Projects: Physically-based shading and Materials system, Physically-based transparency, Morphological antialiasing.

2013, August

Summer School, Achievements and Applications of Contemporary Informatics, Mathematics and Physics, Kyiv, Ukraine.

Stream: Neuroscience

$2009-2015$

Laboratory research assistant, Department of Mathematical Modeling and Informational

Systems, NBICS Centre, National Research Centre "Kurchatov Institute", Moscow, Russia. $2009-2013$

Undergraduate student, B.Sc. in Applied Mathematics and Informatics, Department of Nano-, Bio-, Information and Cognitive Technologies, Moscow Institute of Physics and Technology (State University), Moscow, Russia. 
GPA: 4.4 out of 5.0 .

Thesis: "Modeling of spiking dynamics of biological neural networks in vitro". Grade: 5 out of 5.

\section{Current research interests}

My current research interests lie in the sensorimotor field of computational neuroscience that deals with the models of movement control. My aim is to develop a biophysical model that can control musculoskeletal movement dynamics and adapt to unexpected environmental conditions. My secondary and complimentary interest is in the mechanisms of synaptic plasticity underlying associative memory, comprehension and general cognitive behavior.

\section{List of Publications}

\section{In preparation:}

1. Sobinov A, Boots M, Gritsenko V, et al., and Yakovenko S (2019) Musculoskeletal transformation described with autogenerating multidimensional polynomials.

2. Boots M, Sobinov A, Gritsenko V, et al., and Yakovenko S (2019) Realistic musculoskeletal models for real-time human-in-the-loop control of arm and hand.

3. Sobinov A, Boots M, Gritsenko V, et al., and Yakovenko S (2019) Muscle excitation profiles in complex musculoskeletal model of human hand with muscle-based active joint stiffness.

\section{Peer-reviewed publications:}

1. Yakovenko S, Sobinov A, Gritsenko V (2018) Analytical CPG model driven by limb velocity input generates accurate temporal locomotor dynamics. PeerJ 6:e5849 Available at https://peeri.com/articles/5849.

2. Sobinov A, Boots M, Gritsenko V, Mansouri M, Beringer C, Boninger M, Fisher LE, Collinger J, Gaunt R, Yakovenko S (2018) Decoding muscle activation patterns from kinematics using a detailed musculoskeletal model with active muscle stiffness. Poster abstract at SfN Neuroscience 2018, San Diego, CA, USA.

3. Moon T, Boots M, Sobinov A, Yakovenko S (2018) Realistic biomechanical lowerlimb model implemented for human-in-the-loop applications. Poster abstract at SfN Neuroscience 2018, San Diego, CA, USA.

4. Beringer $C$, Mansouri M, Sobinov A, Boots M, Gritsenko V, Weir D, Simpson T, Fisher LE, Collinger J, Munin M, Boninger M, Gaunt R (2018) Optimizing parameters for model-based control of prosthetic limbs. Poster abstract at SfN Neuroscience 2018, San Diego, CA, USA.

5. Mansouri M, Beringer C, Yakovenko S, Gritsenko V, Sobinov A, Boots M, Munin M, Boninger M, Fisher LE, Collinger J, Gaunt R (2018) A clustering approach to identify the locations of intramuscular electromyographic electrodes used for prosthetic control. Poster abstract at SfN Neuroscience 2018, San Diego, CA, USA.

6. Mansouri M, Beringer C, Gritsenko V, Yakovenko S, Sobinov A, Boots M, Munin M, Boninger M, Fisher LE, Collinger J, Gaunt RA (2018) Differences in kinematics and EMG dimensionality for simple and complex hand movements: implications for prosthetic control. Poster abstract at NIC 2018, Minneapolis, MN, USA. 
7. Sobinov A and Yakovenko S (2017) Model of a bilateral Brown-type central pattern generator for symmetric and asymmetric locomotion. J Neurophysiol:jn.00443.2017, PMID: 29187551, DOI: 10.1152/jn.00443.2017.

8. Sobinov A, Boots M, Gritsenko V, Mansouri M, Beringer C, Boninger ML, Fisher ML, Collinger JL, Gaunt RA, and Yakovenko S (2017) Decoding motor commands for postural control of a biomimetic prosthesis. Presentation abstract at SfN Neuroscience 2017, Washington DC, USA.

9. Boots M, Sobinov A, Gritsenko V, Mansouri M, Beringer C, Boninger ML, Fisher ML, Collinger JL, Gaunt RA, and Yakovenko S (2017) Scaling of musculoskeletal morphometry for human upper-limb models. Presentation abstract at SfN Neuroscience 2017, Washington DC, USA.

10. Mansouri M, Yakovenko S, Gritsenko V, Boots M, Sobinov A, Beringer C, Boninger ML, Fisher LE, Collinger JL, and Gaunt RA (2017) Using biomimetic models and intramuscular EMG for control of myoelectric prosthesis. Presentation abstract at American Society of Biomechanics 2017 conference, Boulder, CO, USA.

11. Sobinov A, Boots M, Hardesty R, Gritsenko V, and Yakovenko S (2016) 'Goodenough' approximation of musculoskeletal dynamics. Poster abstract at SfN Neuroscience 2016, San Diego, CA, USA.

12. Boots M, Hardesty R, Sobinov A, Gritsenko V, Mansouri M, Gaunt R, and Yakovenko S (2016) Optimal solutions of human hand movements using inverse musculoskeletal model. Poster abstract at SfN Neuroscience 2016, San Diego, CA, USA.

13. Sobinov A, Freeman S, and Yakovenko S (2016) Computational modelling of locomotor spinal circuits. Poster abstract at National IDeA Symposium of Biomedical Research Excellence, Washington DC, USA.

14. Sobinov A and Yakovenko S (2015) Analytical solution to leaky integrator model of central pattern generator for locomotion. Poster abstract at SfN Neuroscience 2015, Chicago, IL, USA.

15. Sobinov A (2015) Search for traces of Tataro-Mongolian admixture in genotypes of Eurasian nations. M.Sc. Thesis, Department of Applied Mathematics and Informatics, FNBIC, MIPT, Moscow, Russia.

16. Kukin KA, Sboev AG, and Sobinov AR (2015) Comparison of learning methods of spiking neural networks in different neurosimulators. Proceedings for XVII International Conference "Neuroinformatics-2015", Moscow, Russia. V.1 pp. 134-143.

17. Sobinov A (2013) Modeling of spiking dynamics of biological neural networks in vitro. B.Sc. Thesis, Department of Applied Mathematics and Informatics, FNBIC, MIPT, Moscow, Russia.

18. Sobinov A (2013) Effect of long-term synaptic plasticity STDP on synchronous spiking activity of neural network. Proceedings of 56th MIPT Science Conference, Moscow, Russia. FNBIC department, pp. 16-17.

19.Zendrikov D, Serenko A, and Sobinov A (2013) Phase separating curve in the parameter space of synaptic current. Proceedings of XI Kurchatov School of Young Scientists, Moscow, Russia.

20. Paraskevov AV and Sobinov A (2012) Periodical synchronization of moments of generation of action potentials by two neurons as a result of overabundant summation of 
contributions in synaptic current. Proceedings of 55th MIPT Science Conference, Moscow, Russia. Department of Molecular and Biological Physics, pp. 46-47.

21. Sobinov A, Drizhuk D, and Paraskevov A (2012) Problem of topological connectivity of big biological simulated neural networks. Proceedings of SchoolConference of Young Scientists "Biology-2012", Pushchino, Russia. p. 81.

22. Drizhuk D, Klochikhin V, and Sobinov A (2011) Possibilities of modeling of biological neural networks with NEST. Proceedings of IX Kurchatov School of Young Scientists, Moscow, Russia.

\section{Patents and intellectual property disclosures:}

1. Sobinov A, Yakovenko S, Gritsenko V, Hardesty R, and Boots M (2017) Approximation of complex musculoskeletal dynamics. Provisional Patent Number 62559711, USA.

2. Sobinov AR (2014-03-06) Software for Modeling of Electrical Activity of Biological Neural Networks 'HEM', State Registration Certificate \#2014612785, Russia.

\section{Invited presentations, lectures and posters:}

1. Sobinov A (2019, February) Controlling a prosthetic hand. Seminar at University of Chicago, IL, USA.

2. Sobinov A, Boots $M$, Gritsenko V, Mansouri M, Beringer C, Boninger M, Fisher LE, Collinger J, Gaunt R, Yakovenko S (2018) Decoding muscle activation patterns from kinematics using a detailed musculoskeletal model with active muscle stiffness. Poster presentation at SfN Neuroscience 2018, San Diego, CA, USA.

3. Sobinov A (2018, August) Developing a biomimetic controller for myoelectric prosthesis. Seminar at University of Pittsburgh, PA, USA.

4. Sobinov A, Boots $M$, Gritsenko $V$, Mansouri M, Beringer $C$, Boninger ML, Fisher ML, Collinger JL, Gaunt RA, and Yakovenko S (2017) Decoding motor commands for postural control of a biomimetic prosthesis. Nanosymposium presentation at SfN Neuroscience 2017, Washington DC, USA.

5. Sobinov A (2017) Bionic approach in prosthetics. Research seminar at scientific café QuantROOM and "Cherdak" (Russian News Agency TASS), Moscow, Russia.

6. Sobinov A (2017) Neuroengineering: creation of artificial arm. Scientific seminar at anticafe "Kocherga" lectorium, Moscow, Russia.

7. Sobinov A (2017) Future of neuroengineering: movement without constraints. Series of public lectures at ZIL Culture Center Lectorium and radio interview on "Radio Moskvy" 92FM, Moscow, Russia.

8. Sobinov A (2017) Decoding motor command for control of a myoelectric prosthesis. Symposium presentation at WVU BRNI Neuroscience Retreat, Canaan Valley, WV, USA.

9. Sobinov A, Boots M, Hardesty R, Gritsenko V, and Yakovenko S (2016) 'Goodenough' approximation of musculoskeletal dynamics. Poster presentation at SfN Neuroscience 2016, San Diego, CA, USA.

10. Sobinov A and Yakovenko S (2015) Analytical solution to leaky integrator model of central pattern generator for locomotion. Poster presentation at SfN Neuroscience 2015, Chicago, IL, USA. 
11. Sobinov A (2014, February) Modeling of biological neural networks for ITspecialists. Seminar at Supercomputer Department, FNBICS, NRC "Kurchatov Institute", Moscow, Russia.

12. Sobinov A (2013) Effect of long-term synaptic plasticity STDP on synchronous spiking activity of neural network. Oral presentation at 56th MIPT Science Conference, Moscow, Russia.

13. Sobinov A (2013, March) On the basics of computational neuroscience. Lecture at FNBIC, MIPT, Moscow, Russia.

14. Sobinov A, Drizhuk D, and Paraskevov A (2012) Problem of topological connectivity of big biological simulated neural networks. Oral presentation at International student, postgraduate and young scientist conference «Lomonosov-2012», Moscow, Russia.

15. Sobinov A, Drizhuk D, and Paraskevov A (2012) Problem of topological connectivity of big biological simulated neural networks. Oral presentation at SchoolConference of Young Scientists "Biology-2012", Moscow, Russia.

\section{Awards}

2018: Neuroscience Graduate Student Research Award, WVU RNI. \$500

2018: WVU Rockefeller Neuroscience Institute Trainee Travel Award. \$1000

2016, 2017: West Virginia University Health Sciences Center Research and Graduate Education Office Travel Award. $\$ 400$

2014, February: Igor Vasilyevich Kurchatov Student Award. $\$ 600$

2012 - 2013: Alexandrov Scholarship, Kurchatov Institute. \$300/mo

\section{Participation in projects}

2015 - Present: DARPA HAPTIX (at WVU as Pittsburgh University subcontract)

Our group is focused on developing software for the prosthesis based on a biomechanical model of the human hand. I contribute in the fields of software architecture, musculoskeletal dynamics approximation, decoding of muscle activity patterns.

\section{Repositories}

2018, Fast socket connection between Unreal Engine 4 and MATLAB.

https://github.com/nishbo/unreal matlab connection

2018, Fast socket communication between MATLAB and C server.

https://github.com/nishbo/matlab c sockets

2017, Simulation of Brown-type spinal locomotor central pattern generator. https://bitbucket.org/nishbo/cpg

2016, Collection of MATLAB functions for online streaming data into an HDF5 database file. https://github.com/nishbo/simple-hdf5-vector-streaming

2014, Simple neural network simulation in Python https://github.com/nishbo/simsimpy

2013, C++-based simulation of biological neural network in vitro https://github.com/nishbo/hem v7.0 


\section{Ad hoc reviewer}

2018 - Present: PLOS ONE, $1 \mathrm{~ms} /$ year

\section{Teaching experience}

2018 - Present: Motor Control Journal Club at WVU Neuroscience Department, moderator 2019, spring semester - Research Design and Methods (Exercise Psysiology), seminar 2018, autumn semester - Fundamentals of Neuroscience:

Anatomy of cochlea and encoding of sound

2017, autumn semester - Advanced Neuromechanics, seminars

2017, summer semester - Graduate Medical Neurobiology:

Spinal cord and control of periodic movements

Anatomy and pathways of auditory and vestibular systems

\section{Advising students}

2018, autumn - Maddie Weber, Biomedical Engineering undergraduate student.

2018, autumn - Lindsay Reese, ExPhys undergraduate student. Project: Comparative evaluation of myoelectric prosthesis controllers in live experiments.

2018, summer - Keleigh Cochran, ExPhys undergraduate student. Project: Experimental measurements of active muscle joint stiffness.

2018, summer - Saeed Yousefi, visiting PhD student. Project: Machine learning techniques to predict asymmetricities in gait.

2017, summer - Amanda Barbarossa, SURE BME undergraduate student. Project: Towards virtualized transradial prosthesis with simulated dynamics and surface EMG interface.

2017, summer - Abby Williamson, SURI BME undergraduate student. Project: Musculoskeletal modeling of the lower-limb: a novel approach for locomotor rehabilitation.

\section{Programming Skills}

Python, C, C++, git, Matlab, LaTeX, bash and *nix-systems, Unreal Engine 4, Windows and *nix-based sockets, MPI, MySQL, NEST (NEural Simulation Tool), HTML, CSS, PHP.

\section{List of Select Courses Taken during Undergraduate and Graduate Studies}

Neuroscience-related: Medical Neuroscience, Fundamental Neuroscience, Advanced Neuromechanics, Stroke: from Bench to Bedside, Mathematical Biophysics, Technologies of Artificial Intelligence, Control Theory, Stochastic Processes, Scientific Writing.

Physics-related: Field Theory, Statistical Physics, Quantum theory, Continuum mechanics, Condensed Matter Physics.

Programming-related: Computational Physics, Communication Protocols (TCP), Database Architecture, Compiler Theory, Network and Information Security, Routing, WebTechnologies, Network Programming, Grid and Cloud Computing, Parallel Programming, Parallel Computing and Cluster Analysis. 
Math-related: Mathematical Analysis of Dynamic Systems, Topology, Discrete Mathematics, Continuous Mathematical Models, Group Theory, Partial Differential Equations, Mathematical Problems of Theory of Nanostructures, Functional Analysis, Mathematical Statistics, Mathematical Optimization, Oscillation Theory and Asymptotic Methods. 